Made possible by

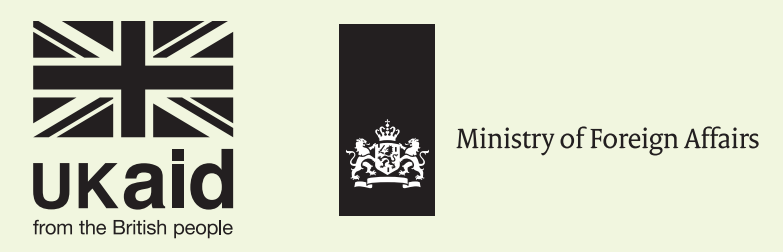




\section{From Unsafe \\ to Safe Abortion \\ in Sub-Saharan Africa \\ Slow but Steady Progress}

Akinrinola Bankole

Lisa Remez

Onikepe Owolabi

Jesse Philbin

Patrice Williams

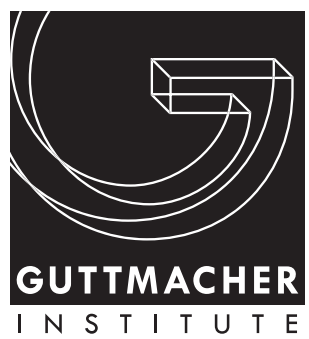




\section{Acknowledgments}

This report was written by Akinrinola Bankole, Lisa Remez, Onikepe Owolabi, Jesse Philbin and Patrice Williams-all of the Guttmacher Institute. It was edited by Michael Klitsch and Haley Ball, and its figures and tables were designed by Michael Moran, also of the Guttmacher Institute.

The authors thank their colleagues Adesegun Fatusi and Susheela Singh for their indispensable comments and insights throughout the project. They are also grateful to the following current and former Guttmacher staff for reviewing an earlier version of this report: Ann Biddlecom, Alanna Galati, Elena Garofalo, Naomi Lince-Deroche, Colette Rose and Sophia Sadinsky. They extend further thanks to Jonathan Bearak for his assistance and advice regarding data on abortion and unintended pregnancy and to Suzette Audam for data analysis.

The authors also express their gratitude to colleagues from other organizations who provided information along the way, especially Tamara Fetters, Ipas; Vladímira Kantorová, United Nations Population Division; Antonella Lavelanet, World Health Organization; and Katherine Mayall, Susan Wnukowska-Mtonga and Margaret Harpin, all with the Center for Reproductive Rights.
In addition, the authors are grateful for the suggestions and advice offered by the following reviewers of the report: Akanni Akinyemi, Obafemi Awolowo University (Nigeria); Janie Benson, independent consultant (USA); Tsungai Chipato, University of Zimbabwe; Ernestina Coast, London School of Economics; Kate Gilles, Population Reference Bureau (USA); Charlotte Hord Smith, independent consultant (USA); Chimaraoke Izugbara, International Center for Research on Women (USA); Patrick G. Christian Ilboudo, Agence de Médecine Préventive (Côte d'Ivoire); Nyovani Madise, African Institute for Development Policy (Malawi); Charles Ngwena, University of Pretoria (South Africa); and Yohannes Wado, African Population and Health Research Center (Kenya).

This report was made possible by a grant from the Dutch Ministry of Foreign Affairs and by UK Aid from the UK Government. The views expressed are those of the authors and do not necessarily reflect the positions and policies of the donors. The Guttmacher Institute gratefully acknowledges the unrestricted funding it receives from many individuals and foundations -including major grants from the William and Flora Hewlett Foundation and the David and Lucile Packard Foundation-which undergirds all of the Institute's work.

Suggested citation: Bankole A et al., From Unsafe to Safe Abortion in Sub-Saharan Africa: Slow but Steady Progress, New York: Guttmacher Institute, 2020, https://www.guttmacher.org/report/ from-unsafe-to-safe-abortion-in-subsaharan-africa. doi:10.1363/2020.32446 


\section{Table of Contents}

Executive Summary

Introduction

(2) Legality of Abortion

3 Abortion Incidence and Trends

4. Abortion Practice and Consequences of Unsafe Abortion

5 Unintended Pregnancy, the Underlying Cause of Abortion

6 Conclusions and Recommendations

Data and Methods Appendix

References 


\section{Executive Summary}

\section{2\% of the region's women of reproductive age live in coun- tries with highly or moderately restrictive abortion laws.}

$\mathrm{T}$ his report on induced abortion in Sub-Saharan Africa offers an overview of the legality of abortion and describes how often and how safely abortions occur in the region's 48 countries. It also examines the underlying context of abortionnamely, unintended pregnancy and its causes. The following are among its principal findings.

\section{Legality of abortion}

- The legality of abortion in Sub-Saharan Africa falls along a continuum from prohibited to allowed without restriction as to reason. As of 2019, $92 \%$ of the region's women of reproductive age live in the 43 countries with highly or moderately restrictive laws. These laws either prohibit abortion altogether or restrict it to cases where a woman's life or health are threatened.

- The African Union's Maputo Protocol is the only human rights instrument with prescriptive language on abortion criteria. It likely contributed to reform in all countries that added legal grounds since the protocol's adoption in 2003.

- Since the protocol was passed, seven countries reformed their laws to meet-and in one case, exceed-the African Union's legal criteria for safe abortion of allowing abortion when the woman's life or physical or mental health is threatened and in cases of rape, incest and grave fetal anomaly.

\section{Incidence of abortion}

- As of 2015-2019, an estimated 33 abortions occur each year per 1,000 women aged 15-49, with little variation across the four subregions of SubSaharan Africa. Overall and subregional rates have been relatively stable over the past 25 years.

- However, given Sub-Saharan Africa's high rate of population growth, an unchanging abortion rate still means increasing numbers of abortions each year. The annual number of abortions nearly doubled between 1995-1999 and 2015-2019, from 4.3 million to 8.0 million.
- Abortion rates in the most populous cities are typically higher than rates in countries as a whole. Moreover, sexually active adolescents have far higher rates of abortion than do all women of reproductive age.

- Global data show that restricting abortion makes no difference in how often it occurs: The annual abortion rate is identical, at 40 per 1,000 women, where abortion is prohibited and where it is broadly legal.

\section{Safety of abortion}

- Abortion is riskier in Sub-Saharan Africa than in any other world region: As of 2010-2014, 77\% of abortions in the region are unsafe, compared with the global average of $45 \%$. The resulting incidence of unsafe abortion-6.2 million per year-exacts a heavy toll on the region's women and families.

- Unsafe abortions are the sum of those that are less safe (done by either an untrained person or with a nonrecommended method) and least safe (done by an untrained person using a nonrecommended method). Roughly half of all abortions in the region qualify as least safe; at 69\%, this proportion is highest in Middle Africa.

- As of 2019, Sub-Saharan Africa has the highest abortion case-fatality rate of any world region, at roughly 185 deaths per 100,000 abortions, for a total of 15,000 preventable deaths every year. Ongoing efforts to both improve the safety of abortion and expand access to quality postabortion care have likely contributed to a two-fifths decline in this rate since 2000 , when an estimated 315 women died per every 100,000 abortions.

\section{Abortion practice and postabortion care}

- Several countries that have expanded the legal grounds for abortion have adopted guidelines modeled on international medical standards and now offer safe, facility-based abortions. However, 
in most Sub-Saharan countries, legal restrictions and stigma still compel women to undergo clandestine abortions, the safety of which cannot be ensured.

- The growing use of misoprostol alone to induce abortion is probably making clandestine abortions safer. This scenario has been documented in Latin America, a region with highly restrictive abortion laws, where use of misoprostol became widespread decades ago.

- Another strategy that can improve outcomes in low-resource settings is to shift postabortion care to midlevel personnel such as nurses and midwives, who are much more numerous than physicians. Postabortion care is always legal, and medical personnel have an ethical duty to provide it.

- Much remains to be done to transition away from dilation and curettage, a highly invasive and costly method of postabortion care, toward the use of misoprostol and vacuum aspirationmethods that meet international guidelines.

\section{Unintended pregnancy and abortion}

- Nearly all abortions result from unintended pregnancies. Sub-Saharan Africa has the highest unintended pregnancy rate of any world region, at 91 per 1,000 women-primarily because the region has the highest pregnancy rate overall (218 per 1,000). The proportion of pregnancies reported as unintended is actually lowest in Sub-Saharan Africa, at $42 \%$.

- Once women become pregnant without intending to be, an estimated 37\% interrupt such pregnancies. This proportion has risen significantly over the past three decades. Such an increase could seriously affect the well-being of women who face barriers to safe abortion. At the regional level, social stigma remains a persistent barrier; at the national level, barriers can include highly restrictive laws or the inadequate implementation of liberal laws.

- The desire for smaller families is increasing, but in order to have smaller families, women need access to modern contraceptives to limit and space their pregnancies. Should this not occur, many will have unintended pregnancies that they then resolve through abortion. Many of these abortions will be clandestine, and thus likely unsafe, unless the strong stigma attached to abortion-which is reflected in the region's restrictive laws_-is addressed.
The way toward legal and safer abortion is clearby reducing stigma and expanding legality. But these are only first steps. Reform must be accompanied by political will and full implementation of the law, so that all women-despite inability to pay or fear of stigma-can seek and receive a legal, safe abortion.

In countries with few or no legal grounds for abortion, mitigating the harm of unsafe abortion becomes the priority. The declines in abortionrelated deaths demonstrate the benefits of expanding the reach and quality of postabortion care. The increasing use of misoprostol to induce abortion is likely also saving lives. Clandestine abortion can be made safer through harm reduction initiatives that have worked elsewhere, such as disseminating accurate information on how to use misoprostol. In every Sub-Saharan African country, expanding modern contraceptive use will go a long way toward reducing unintended pregnancies and subsequent unsafe abortions.
In countries with few or no legal grounds for abortion, mitigating the harm of unsafe abortion becomes the priority. 


\section{(1) Introduction}

M any of the 48 nations of Sub-Saharan Africa are undergoing broad societal transformation. Progress is being made on many fronts, including improvements in the standard of living, political stability, Internet connectivity and educational attainment. As the economies of the subcontinent continue to grow, so do expectations for parallel positive developments in women's health. However, one of the major contributors to poor health among women is the continuing high prevalence of unsafe abortion in the region. Of all world regions, Sub-Saharan Africa has the highest estimated proportion of abortions classified as least safe (i.e., done by an untrained provider using a nonrecommended method). ${ }^{2}$ Such abortions have the highest likelihood of being incomplete or leading to medical complications, either of which can result in the need for immediate medical treatment. Yet this risk to the health and survival of women of all ages is largely preventable.

There is no medical reason why abortion should be unsafe. Indeed, when abortions are done following internationally accepted standards, they very rarely result in complications. ${ }^{3}$ They only become unsafe when the lack of access to safe services-often, though not always, determined by legal restrictions - leads women to end a pregnancy under conditions that pose a risk to their health. Most SubSaharan countries that highly restrict abortion have penal codes dating from the colonial era. ${ }^{4}$ But even in the few countries in the region that allow abortion on broad grounds, access to safe and legal services is often limited, for a range of reasons. Foremost among these reasons are poor implementation of the law, religious and social stigma, and lack of health care resources and of trained personnel.

The safety of abortion is closely related to its legal a) Because the six Northern African countries differ considerably in the contextual factors that underlie and explain unsafe abortion, this report focuses solely on the 48 Sub-Saharan countries. status. ${ }^{5,6}$ Abortion incidence, however, is not related to legality. For example, the global abortion rate is identical-at 40 abortions each year per 1,000 women aged 15-49-where abortion is broadly legal and where it is prohibited. ${ }^{7}$ By comparison, the prevalence of the least-safe abortions increases dramatically with legal restrictiveness: Fewer than $1 \%$ of abortions are classified as least safe in countries with the least-restrictive laws, compared with $31 \%$ of those in countries with the most-restrictive laws. ${ }^{5}$ Because the vast majority of Sub-Saharan African countries have restrictive abortion laws, the region bears a disproportionate burden of unsafe abortion: Whereas $45 \%$ of the world's abortions in 2010-2014 were estimated to be unsafe, that proportion reached $77 \%$ in SubSaharan Africa. ${ }^{2}$

When women cannot openly and legally obtain an abortion, they have whatever type of abortion is available to them. Such informal services often lead to complications and an immediate need for facility-based care. Not receiving this care in time, or receiving inadequate care, can result in avoidable injury and death. Among all of the world's women, Sub-Saharan African women are the most likely to die from abortion: As of 2019, the region has the highest abortion-related case-fatality rate of any world region. ${ }^{8}$ Unless Sub-Saharan governments act to prevent avoidable maternal deaths from unsafe abortion, they will be hard pressed to meet the United Nations Sustainable Development Goal target (in Goal 3) of reducing the maternal mortality ratio to fewer than 70 maternal deaths per 100,000 live births by $2030 .^{9}$ As of this writing, the precise impact of the COVID-19 virus on reproductive health is unknown, but it is likely to further complicate meeting that goal.

All Sub-Saharan African countries are member states

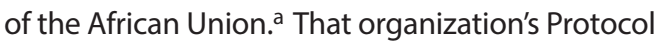
to the African Charter on Human and Peoples' Rights on the Rights of Women in Africa is the main legal instrument for protecting women's rights in Africa. It was also the first official human rights document to recognize abortion "under certain conditions" as women's human right. ${ }^{10}$ Known as the Maputo 


\subsection{Sub-Saharan African subregions and countries}

\section{Western Africa}

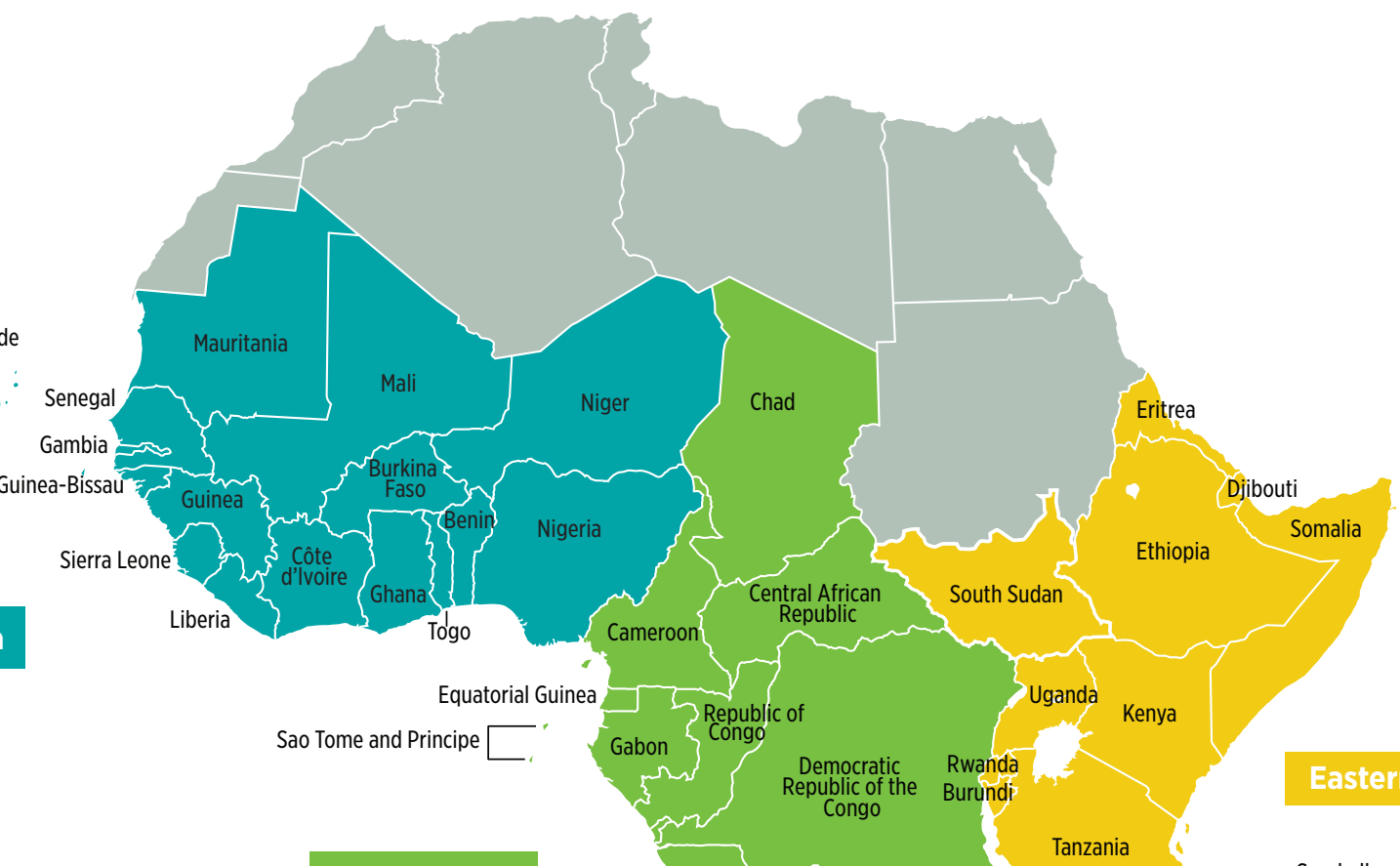

Middle Africa

Southern Africa

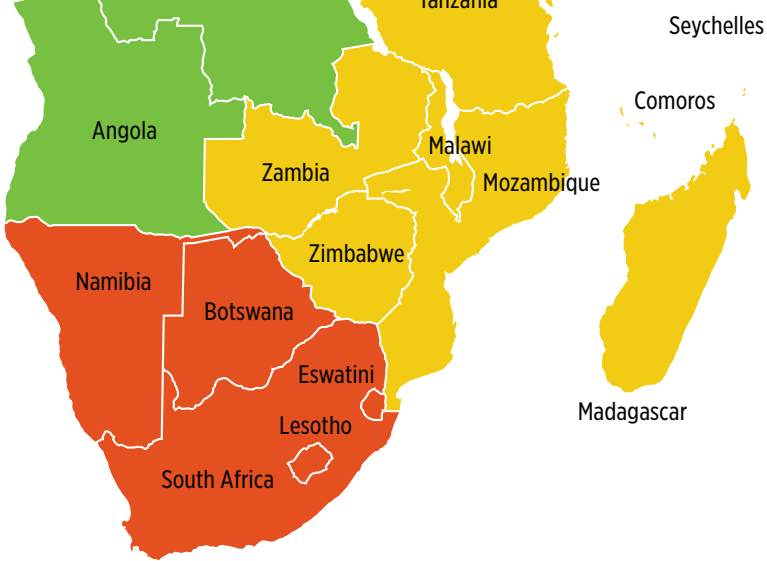

Mauritius

Note: Countries are organized by subregion according to the United Nations Statistics Division. Source: reference 13.

Protocol, it stipulates in Article 14(2)c that to protect women's health and reproductive rights, safe abortion should be authorized when continuing the pregnancy endangers the woman's life; when her physical or mental health is threatened; when the pregnancy results from sexual assault, rape or incest; and when the fetus has a grave anomaly (Box 1.1, page 8$)^{11}$

This report in part responds to the call in the Maputo Plan of Action to reduce the incidence of unsafe abortion by "compil[ing] and disseminat[ing] data on the magnitude and consequences of unsafe abortion..12 Such data are hard to come by, given that both abortion seekers and abortion providers are understandably reluctant to disclose information about a procedure that is often legally restricted and highly stigmatized. To fill this important data gap, researchers have been continually innovating to better measure deliberately hidden behaviors. We draw from a few of these methodologies (see Data and Methods Appendix, page 39) to present information on how often women in Sub-Saharan Africa resort to abortion and the extent to which those abortions lead to the need for and receipt of postabortion care. 
$\mathrm{BOX}$

\section{Excerpts from the Protocol to the African Charter on Human and Peoples' Rights on the Rights of Women in Africa}

\section{Article 14: Health and Reproductive Rights}

1. States Parties shall ensure that the right to health of women, including sexual and reproductive health is respected and promoted....

2. States Parties shall take all appropriate measures to:...

c) protect the reproductive rights of women by authorising medical abortion in cases of sexual assault, rape, incest, and where the continued pregnancy endangers the mental and physical health of the mother or the life of the mother or the foetus.

Note: In this context, “medical abortion” denotes safe abortion. Source: reference 11.

b) As defined by the United Nations Statistics Division.

\section{Structure of the report}

This first broad summary report on abortion in Sub-Saharan Africa presents an overview of abortion safety and consequences in the region and describes how abortion fits into the broader context of fertility preferences and unintended pregnancy. Its goal is to synthesize what is known and to provide an evidence base for improving health services and policies throughout the region. We present data for the region overall and for each of its four subregions:b Eastern Africa (18 countries), Middle Africa (nine countries), Southern Africa (five countries) and Western Africa (16 countries; Figure
1.1, page 7)..$^{13}$ These are the most recent data available on abortion levels and trends in these subregions, along with their diverse legal contexts, the nature of abortion practices and service provision, and factors contributing to unintended pregnancy.

Chapter 2 categorizes countries by legal status of abortion. In general, the law provides a useful indicator of whether women will have access to safe, legal abortion or will be compelled to seek out clandestine abortions, which are far more likely to be unsafe. Chapter 3 presents estimates of abortion incidence for the region and for each subregion and discusses trends in abortion incidence over the past few decades. That chapter also covers evidence on abortion incidence for countries where national incidence studies have been conducted.

Chapter 4 outlines the current practice of abortion and details the consequences of unsafe abortion. Such consequences are visible in the need for and use of postabortion care, which the chapter also quantifies and describes. Chapter 5 provides information on the interplay between fertility preferences and contraception that drives the incidence of unintended pregnancy. Specifically, that chapter examines regional and subregional variations in modern contraceptive use and in unmet need for modern contraception. Chapter 6 summarizes the report's main findings and proposes recommendations and ways to address unsafe abortion to improve women's health throughout the region. 


\section{Legality of Abortion}

$T^{\text {Tes }}$ he safety of abortion depends in part on the extent to which it is legal. In highly or moderately restrictive settings, the general lack of formal abortion services, together with the desire to avoid judgment and arrest, drives women to seek clandestine services, which often do not follow best practices. In addition, the gap between what the law says and what actually occurs is often large: On the one hand, restrictive abortion laws may be minimally applied; on the other hand, barriers can obstruct access to safe abortion in countries with broadly liberal laws. Because we cannot report on how the implementation of the law differs from its wording in 48 countries, we are limited to what is readily available - the letter of the laws - and a literal reading of them.

Much of Sub-Saharan Africa follows a common paradigm in which penal or criminal codes dictate the circumstances (if any) under which abortion is legal. ${ }^{14}$ The codes for countries with absolute bans simply specify the punishments for those seeking or providing abortions; for nearly all remaining countries that criminalize abortion, penal codes word the legal grounds as exceptions to when abortion is punishable. However, as of 2019, one Sub-Saharan country, Zambia, allows abortion on any health or socioeconomic ground, ${ }^{15}$ and four (Cabo Verde, ${ }^{16}$ Guinea-Bissau, ${ }^{17}$ Sao Tome and Principe $^{18}$ and South Africa ${ }^{19}$ ) have decriminalized abortion, allowing it without restriction as to reason, with procedural requirements.

In addition, legal instruments with the force of national law-such as constitutions, judicial decisions, health codes and reproductive health laws-can contain provisions concerning abortion. Complementary sources of criteria, such as ministerial guidelines or codes of medical ethics, can also guide implementation of the law. Indeed, at times they may conflict with it; should this happen, laws will always take precedence over guidelines or ethics codes in establishing legal criteria. We provide here a broad overview of abortion legality as of 2019 for all 48 Sub-Saharan countries. We describe notable legal changes since 2000 and discuss a few strategies that have-or have notworked to bring about such changes.

\section{Legal grounds for abortion as of $\mathbf{2 0 1 9}$}

We classify abortion laws according to a widely used rubric of six mutually exclusive categories (see Data and Methods Appendix, page 39) that range along a continuum from the most restrictive to the least restrictive. ${ }^{20}$ Laws that completely prohibit abortion fall into Category 1. After Category 2, which permits abortion to save a woman's life, each subsequent category includes the grounds in prior categories and adds another legal ground, up to Category 6, in which abortion is legal without restriction as to reason. For expedience, we have collapsed these six categories into three overarching groupings of countries by the nature of their abortion law: countries with highly restrictive laws (in Categories 1 and 2), those with moderately restrictive laws (in Categories 3 and 4) and those with broadly liberal laws (in Categories 5 and 6). Because the 48 nations differ dramatically in population size, we distribute both the region's number of countries and its number of women of reproductive age (15-49) into these six mutually exclusive categories of legality. Many countries have other grounds under which abortion is allowed-including, but not limited to: rape, incest and grave fetal anomaly - that do not contribute to a country's placement along this continuum.

In terms of the six continuum categories of legality, of the region's 48 countries, 16 (or 33\%) have highly restrictive abortion laws (Table 2.1, page 10). ${ }^{20-23}$ Six of these countries prohibit abortion altogether (Category 1), and 10 permit it when a woman's life is in danger (Category 2). Another 27 countries (56\% of countries in Sub-Saharan Africa) have moderately restrictive abortion laws-15 allowing the procedure when continuing the pregnancy would
Since the turn of the millennium, 21 Sub-Saharan countries have reformed their penal codes or passed laws to expand the legal grounds for abortion. 


\subsection{Sub-Saharan African countries, by subregion, according to legal status of abortion}

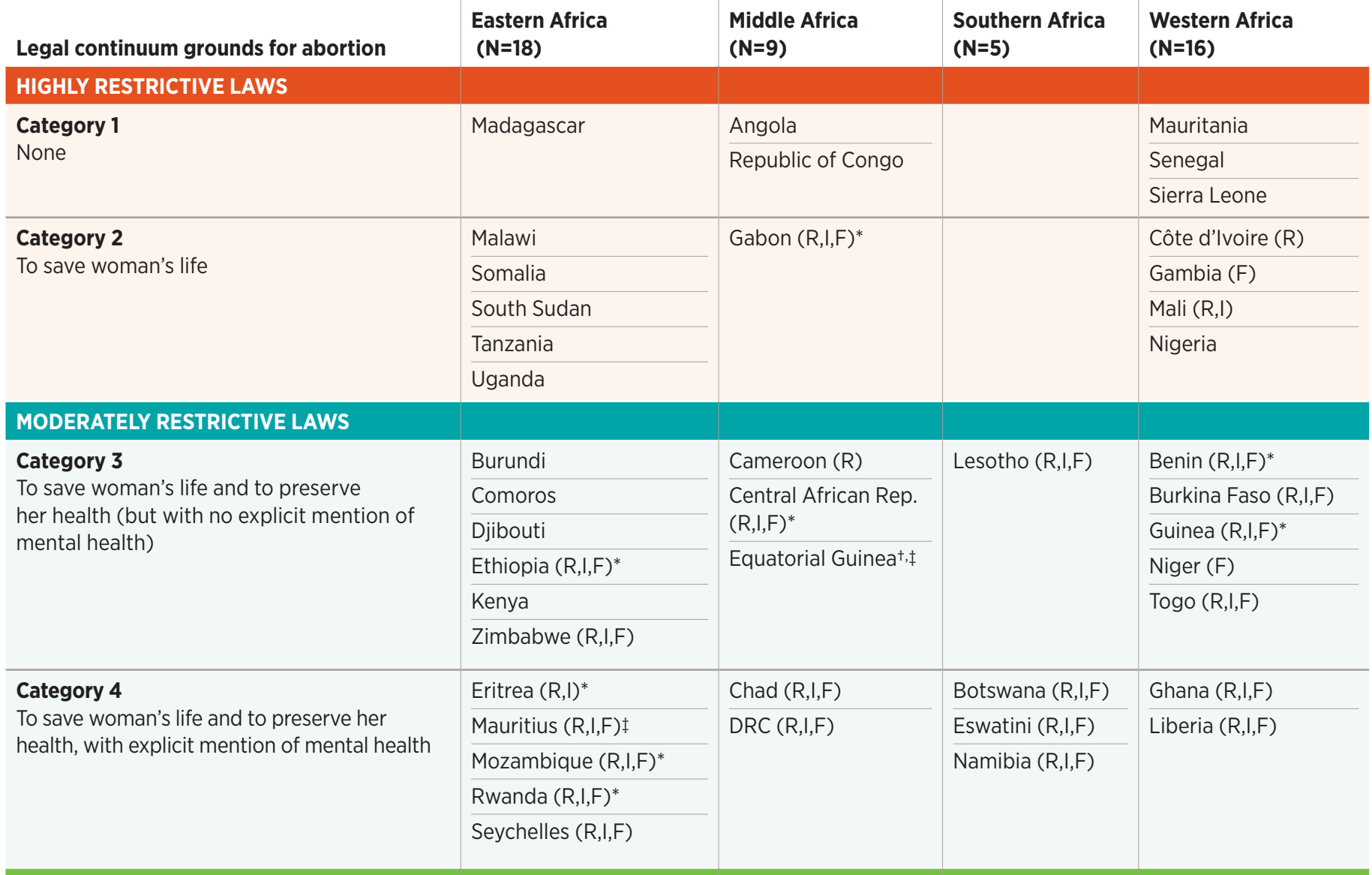

\section{BROADLY LIBERAL LAWS}

\section{Category 5}

To save woman's life and to preserve her physical/mental health, plus socioeconomic grounds

\section{Category 6}

No restriction as to reason

(with gestational and other requirements)

\section{Most common additional legal grounds:}

$\mathrm{R}=$ rape

I=incest

$F=$ grave fetal anomaly

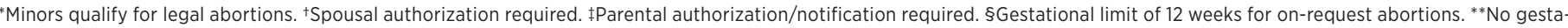
tional limit specified for on-request abortions. Notes: Many laws providing grounds for legal abortion are independent from a country's categorization on the legal continuum. Most

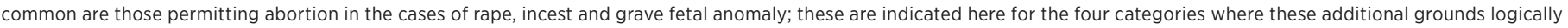
apply. DRC=Democratic Republic of the Congo. Sources: references 20, 22, 23 and 90. 
endanger a woman's general health (but with no explicit mention of mental health; Category 3) and 12 also allowing it when her mental health, in particular, would be compromised (Category 4; see the Data and Methods Appendix, for a full discussion of both health categories). Finally, five Sub-Saharan African countries (10\%) permit abortion on broad legal grounds: One allows abortion on survival, health and socioeconomic grounds (Category 5); and four permit abortion without restriction as to reason, within specified gestational limits that often correspond to specific legal grounds (Category 6).

Of the 255 million women of reproductive age in Sub-Saharan Africa, ${ }^{24} 45 \%$ live under highly restrictive laws, $47 \%$ live where laws are moderately restrictive and $8 \%$ live where abortion is broadly legal (Figure 2.1). ${ }^{20-23}$ The combined total of $92 \%$ living under highly or moderately restrictive abortion laws is in part a legacy of colonialism, although each of the former colonial powers has since fully decriminalized abortion. ${ }^{20}$ Indeed, a few SubSaharan countries still have-or have only recently reformed-penal codes dating from the 19th century. For example, Sierra Leone is one of several former British colonies that inherited England's
1861 Offences Against the Person Act, ${ }^{25}$ but it is the only one where that code is still in effect.

The enormous range in populations across these 48 countries (from just 23,000 women of reproductive age in Seychelles to 46 million in Nigeria ${ }^{24}$ ) means that nations with especially large populations exert outsized influence within their subregion. For example, $71 \%$ of women aged 15-49 in Western Africa live where abortion is highly restricted because one Category 2 country, Nigeria, accounts for most of that subregion's total population. Similarly, 77\% of women in Middle Africa live where abortion is moderately restricted because the populous Democratic Republic of the Congo (DRC) is in Category 4. Finally, Southern Africa presents the most unequal distribution by legality: Since this subregion's relatively small population is overwhelmingly dominated by South Africa, a very large majority of reproductive-age women (88\%) live where abortion is broadly legal. South Africa, and thus by extension, the subregion of Southern Africa, also stands out on many other measures; see Appendix Table 1 (page 43) for a range of indicators that were available for 37 countries.

FIGURE

2.1 The vast majority of women of reproductive age live where abortion is highly or moderately restricted.

\section{$\%$ of women aged 15-49}

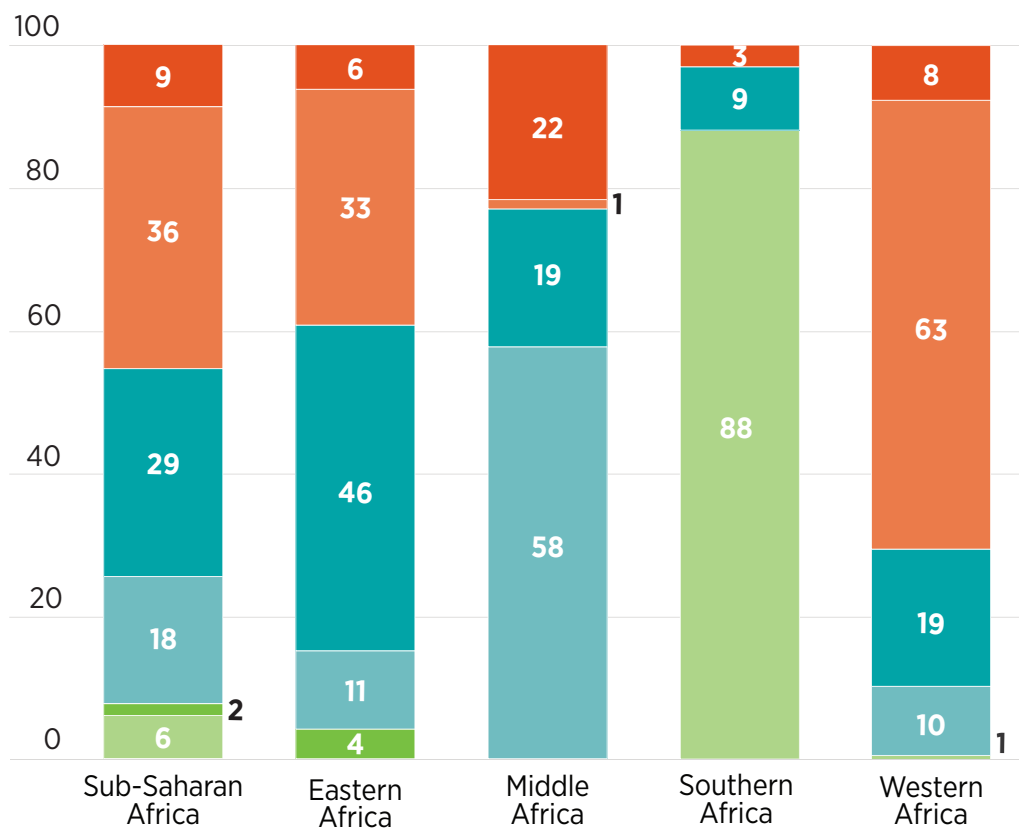

Legal continuum grounds for abortion

Category 1. None

Category 2. To save woman's life

Category 3. To save woman's life and to preserve her health (but with no explicit mention of mental health)

Category 4. To save woman's life and to preserve her health, with explicit mention of mental health

Category 5. To save woman's life and to preserve her physical/mental health, plus socioeconomic grounds

Category 6. No restriction as to reason

Note: Countries in Categories 1 and 2 have highly restrictive abortion laws; those in Categories 3 and 4 have moderately restrictive laws; and those in 5 and 6 have broadly liberal laws. Sources: references 20-24. 
As previously mentioned, countries may permit abortion on a number of grounds that do not affect their overall placement along the legal continuum but that can meaningfully expand access to legal abortion in otherwise highly or moderately restrictive settings. The three most common of these are also specifically mentioned in Article 14 of the Maputo Protocol: rape, incest and grave fetal anomaly. ${ }^{11}$ These additional grounds for abortion apply only to countries in Categories 2-5, as they logically do not involve countries with absolute bans or those that already allow abortion without restriction as to reason. Of the 38 countries in Categories 2-5, 20 (53\%) allow abortion for all three of the most common additional criteria. These 20 countries are home to some 90 million women of reproductive age. All of the relevant countries in Southern Africa and half of those in Western Africa allow abortion on these three additional grounds.

Actual access to safe abortion on any of these three additional grounds is by no means guaranteed, however. For example, in the case of pregnancies resulting from rape, several countries require onerous burdens of proof, such as submitting an affidavit to a magistrate (in the case of Zimbabwe ${ }^{26}$ ) or requiring that a magistrate attest to the materiality of the facts (in Cameroon ${ }^{27}$ and Burkina Faso ${ }^{28}$ ). It is also worth noting in this context that Middle Africa and Southern Africa have the highest prevalence of nonpartner sexual violence ${ }^{c}$ of any world subregions ( $21 \%$ and $17 \%$, respectively, compared with a global average of $7 \%$ ). ${ }^{29}$ Furthermore, rape is often wielded as a weapon of war in the long-running civil conflicts in the region. ${ }^{30-32}$

Nearly all Sub-Saharan countries link specific legal grounds to gestational limits. For example, in Mozambique (Category 4), abortions needed to ensure a woman's survival or health are allowed through 12 weeks of pregnancy; the limit then extends to 16 weeks for pregnancies resulting from rape or incest and to 24 weeks for severe fetal anomalies. ${ }^{21}$ For South Africa (Category 6), a woman does not have to cite a specific reason for seeking an abortion in the first 12 weeks of pregnancy; she is entitled to a legal abortion through 20 weeks if she satisfies one of the health or socioeconomic criteria and beyond 20 weeks if continuing the pregnancy threatens her life or if the fetus has severe anomalies. ${ }^{19}$

\section{Recent legal reform}

Since the turn of the millennium, 21 Sub-Saharan countries have reformed their penal codes or passed laws to expand the legal grounds for abortion (Figure 2.2, page 13). This total is made up of 14 that both changed categories and began allowing abortion on grounds of rape, incest or fetal anomaly; four that changed categories only; and three that did not change categories but enacted an additional ground. Notably, 13 countries moved away from absolute bans since 2000. Seven countries-Chad, DRC, Eswatini, Mauritius, Mozambique, Rwanda and Sao Tome and Principe-moved to comply with the grounds set out in the Maputo Protocol's Article 14. In other words, they are in Category 4 (with all three additional grounds of rape, incest and grave fetal anomaly) or higher. Sao Tome and Principe's 2012 penal code reform moved the country across the entire legality continuum, ${ }^{18}$ something no other country in the world has done except Nepal. ${ }^{6}$

\section{The lengthy process of legal reform}

Efforts to expand the legal criteria for abortion are arduous and are characterized by incremental, painstaking progress and setbacks. Advocacy that unites a broad coalition of national actors from the legal, medical and rights perspectives is often a prerequisite for influencing politicians to reform national laws.

South Africa offers an example of how coalition building, together with seizing a historic moment, led to decriminalization in 1996. The African National Congress, which shepherded the country's transition to democracy after apartheid, highlighted the unequal impact of unsafe abortion on Black South African women when pressing for legal reform. ${ }^{33}$ Advocacy by highly placed national politicians or dignitaries can also help to overcome the difficulties inherent in reforming abortion laws. For example, the Eastern African island nation of Mauritius reformed its law in 2012, in part due to the involvement of a Mauritian-born member of the monitoring body that is tasked with ensuring compliance with the Convention on the Elimination of All Forms of Discrimination against Women, the CEDAW Committee. ${ }^{34,35}$

\section{National-level laws}

Penalties for abortion and the grounds under which it is and is not punishable are most commonly established in penal codes. Many SubSaharan African countries also define legal grounds 


\subsection{A total of 21 Sub-Saharan countries expanded legal grounds for abortion between 2000 and 2019.}

\section{Legal continuum grounds for abortion}

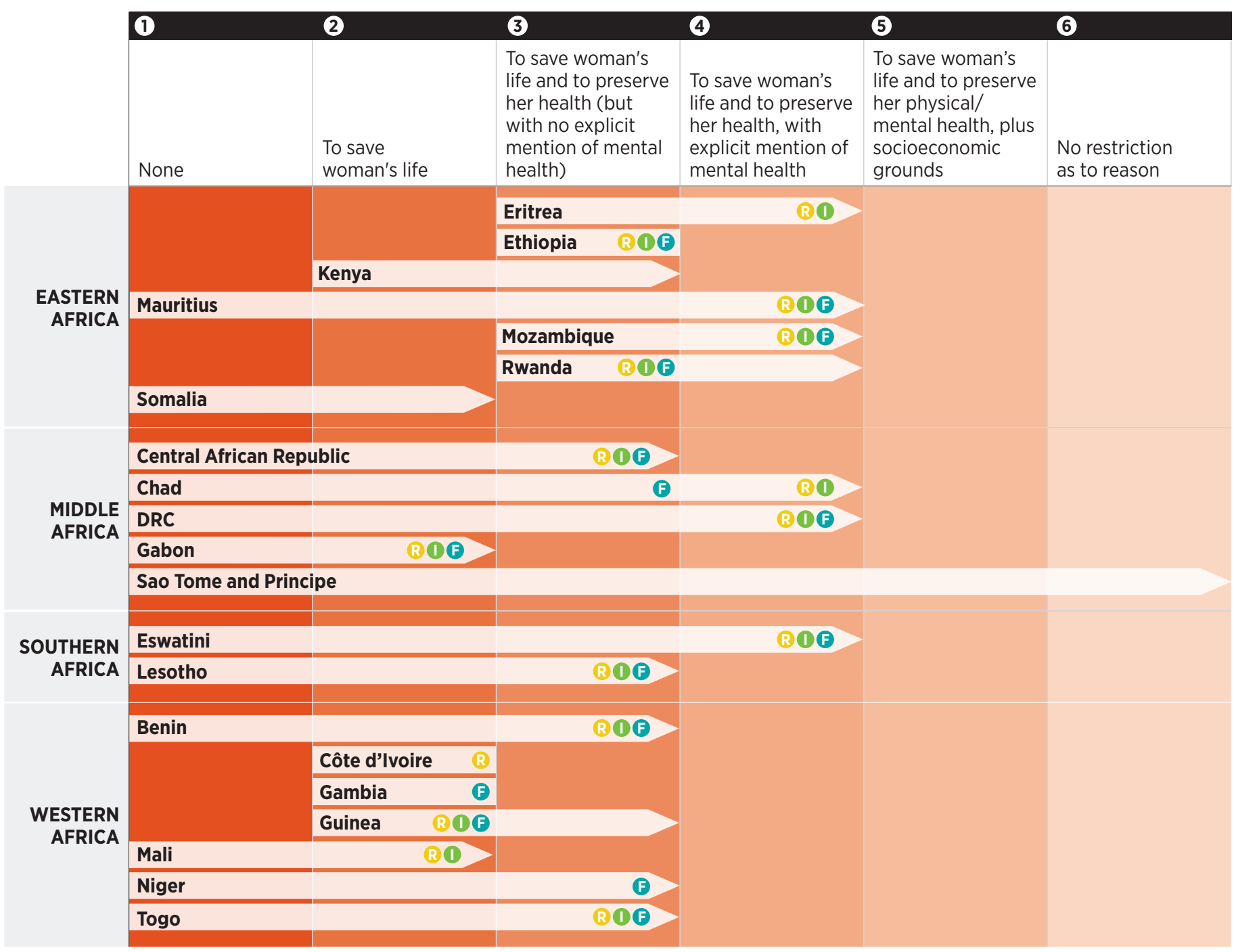

Most common additional legal grounds:
(R) Rape
(1) Incest
P Grave fetal anomaly

Notes: Many laws providing grounds for legal abortion are independent from a country's categorization on the legal continuum. Most common are those permitting abortion in the cases of rape, incest and grave fetal anomaly; these are indicated here for the four categories where these additional grounds logically apply. The symbols for these additional grounds are placed above so as to correspond with a country's continuum category at the time the additional ground was enacted. DRC=Democratic Republic of the Congo. Sources: references 20 and 226 ; national laws not covered in these two secondary sources are available from the authors. 
The impact of Article 14 of the African Union's 2003 Maputo Protocol cannot be overstated.

d) Benin, Burkina Faso, Central African Republic, Chad, Guinea, Guinea-Bissau, Mali, Niger and Togo.

e) Burundi, Central African Republic, Chad, Eritrea, Madagascar, Niger, Somalia and South Sudan. for abortion in national reproductive health laws. These tend to follow a single template. The writing and passage of these laws likely grew out of an international effort to harmonize reproductive health policies and services with international conventions and agreements. ${ }^{36}$ Eleven SubSaharan countries have passed reproductive health laws, and nine of them specify grounds for legal abortion ${ }^{d}$ (the exceptions are Senegal ${ }^{37}$ and Madagascar ${ }^{38}$ ). A few of these laws have-or, at the time they were enacted, had-more legal grounds for abortion than were specified in penal codes.

Several countries have included abortion language in laws meant to strengthen women's rights and address gender inequality. For example, Gambia passed its first Women's Act in 2010. That act promises to protect women's "reproductive health rights" by authorizing safe abortion when continuation of pregnancy threatens the woman's life or when the fetus has a grave anomaly, and by paying for the abortion if the woman cannot. ${ }^{39}$

Constitutional amendments present yet another national-level pathway to abortion reform. Kenya and Somalia exemplify change achieved as part of a broad package of constitutional reforms. Kenya's 2010 constitutional reform, which was put to a popular referendum, included language permitting abortion when "there is a need for emergency treatment, or the life or health of the woman is in danger, or if permitted by any other written law." ${ }^{40}$ In 2012, Somalia's new constitution authorized abortion in cases of necessity, especially to save the life of the pregnant woman. ${ }^{41}$

International treaties and protocols The impact of Article 14 of the African Union's 2003 Maputo Protocol cannot be overstated. As of October 2019, 39 Sub-Saharan countries had ratified the protocol. ${ }^{42}$ Of the remaining nine countries in the region, one has not signed the protocol (Botswana), and eight have signed but not ratified

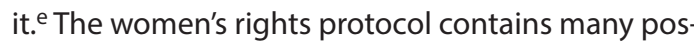
itive provisions for empowering women, including directing member states to ensure women's rights in the spheres of marriage, fertility and contraceptive use, harmful traditional practices and prevention of HIV infection and sexual violence, among others. ${ }^{11}$ The African Union is unique in setting conditions for legal abortion in a rights charter. According to the African Union's General Comment on Article 14, to ensure that the legal abortions required by the protocol are widely accessible, countries must provide the necessary infrastructure for safe services and mechanisms for disseminating information about their availability. ${ }^{10}$

The 2018 reform in the DRC is the latest example of how Article 14 can be leveraged to effect change. That country, which accounts for nearly half of Middle Africa's population, ${ }^{24}$ made Article 14 provisions into domestic law by publishing the protocol and invoking an article in the constitution that elevates international treaties over national law. ${ }^{43}$ A 2018 circular issued by the president of the Constitutional Court stipulates that medical facilities are obligated to safely provide abortions sought on the grounds established by the Maputo Protocol. Further, criminal or judicial authorities cannot prosecute the providers or women involved. ${ }^{44}$

Rwanda provides another notable example: That country first had to remove its reservation to the protocol before adding rape, incest and fetal anomaly as legal grounds for abortion in $2012 .^{45,46}$ Seven years later, the Ministry of Health issued an order to regulate safe abortion that, in its preamble, affirms that the World Health Organization (WHO) definition of health applies. Since WHO's constitution defines health as a "state of complete physical, mental and social well-being," ${ }^{\prime \prime 7}$ the country now complies with all Article 14 grounds. ${ }^{23}$

\section{Widening the interpretation of existing laws}

Rather than extending access through the law per se, several relatively restrictive countries have implemented the law expansively by issuing and enforcing medical guidelines modeled on the WHO publication Safe Abortion: Technical and Policy Guidance for Health Systems (Box 2.1, page 16). Those guidelines lay out internationally recognized best practices for safe abortion provision. ${ }^{3}$ Ethiopia's use of the approach of broadly interpreting a restrictive law is noteworthy. So is Ghana's: That Western African country updated its standards and protocols in 2012 to clarify that no evidence is needed for legal abortions in cases of rape or threat to the woman's mental health. ${ }^{48}$ That no proof of the crime of rape is needed is also specified in African Union guidance on enacting this ground. ${ }^{10}$

\section{Obstacles to ensuring access to legal abortion}

Successful reform is sometimes achieved only after decades of unsuccessful attempts. Malawi and Sierra Leone are examples of countries currently in the long process of marshalling domestic advocacy, international influence, evidence of 
harm to public health and political will to achieve legal reform. In July 2015, the Malawi Law Reform Commission proposed the Termination of Pregnancy Bill to the Malawian Parliament; this bill-the culmination of many years of effort by an array of stakeholders-would have aligned Malawian law with the Maputo Protocol. ${ }^{49}$ However, the bill stalled, and lawmakers have not yet debated it. Similarly, in Sierra Leone, the Safe Abortion Act, which would have allowed abortion without restriction as to reason, was passed by the parliament twice (in 2015 and 2016). ${ }^{50}$ However, the president at the time refused to sign it, leaving in place an outright ban that dates from the Victorian era. ${ }^{25}$

Law reform alone far from guarantees that women will get the safe procedures that they are legally entitled to. Implementation of a law matters far more than its wording, and many obstacles prevent new laws from changing entrenched behaviors. Failure to inform the public, health care providers and government officials about what a new law entails is a common barrier to implementation. The experience of Zambia shows how, in the absence of public information campaigns and the newly legal services being out in the open, decades may pass before this information reaches all interested parties. ${ }^{51}$ For example, in 2014, only $16 \%$ of Zambian women in three provinces knew the legal criteria for abortion that had been in place since 1972. ${ }^{52}$ Research from 2005 indicates that a generalized lack of knowledge about the South African abortion law contributed substantially to the high proportion of abortions obtained outside of formal facilities there, despite nearly a decade since decriminalization..$^{53}$ In Ghana, only $11 \%$ of women of reproductive age who had heard of abortion knew the grounds on which it was legal in the country in $2017,{ }^{54}$ up from just $4 \%$ in $2007 . .^{55}$

Laws that require authorization from multiple physicians can block women's access to safe abortions where severe shortages of physicians make such requirements impractical or effectively impossible; this is the case throughout Sub-Saharan Africa. ${ }^{56} \mathrm{~A}$ solid majority $(60 \%)$ of Sub-Saharan Africans reside in rural areas, ${ }^{57}$ which have few medical professionals of any type. The relatively liberal Zambian law, for example, requires three physicians-one of whom must be a specialist - to sign off on a nonemergency legal abortion. ${ }^{15}$ This requirement remains in effect ${ }^{58}$ despite the country's having fewer than 12 physicians per 10,000 inhabitants as of $2018 .^{56}$ Women's care is delayed while obtaining triple authorization; many women do not even try to obtain it, and others try, give up and then end up having a potentially unsafe abortion. ${ }^{59}$ Delays in abortion care can lead to increases in the risk of complications and in costs, as well as greater difficulties in finding a provider, since comparatively few specialists are trained in later abortions. Moreover, very lengthy delays can place women outside the legal gestational limit, forcing them to have an unwanted birth.

Given the general scarcity of trained medical professionals, actions that further deplete their availability are especially damaging. This is the case with conscientious objection, in which a provider's right to refuse to provide abortion on moral and religious grounds has to be balanced with a woman's right to a legal service. Where conscientious objection is unregulated, its overuse can shrink the pool of personnel who are available to perform a legal service. In South Africa, for example, the law specifies that only the person who would be performing the abortion is allowed to opt out of doing so. Yet public-sector health units often have to hire private nurses to replace staff who illegally opt out of participating in an abortion. ${ }^{60}$

Religiously affiliated nongovernmental organizations (NGOs) are an important source of care in many places, but they can be much more likely than other facilities to refuse to provide legal abortion care. In Northern Ghana, ${ }^{61}$ conscientious objection by workers at religious NGOs creates a patchwork of availability, depending on which type of facility women happen to live closest to and are able to afford. Evidence from Zambia demonstrates that practitioners who refuse to provide abortion sometimes base their decision to refer a woman to a willing provider-which the law requires-on a subjective assessment of the merits of her request for a legal abortion. ${ }^{62}$ Such mandated referrals often are so poorly or cryptically worded that they end up being useless, turning the legal guarantee that a colleague has to perform the abortion into an empty promise.
Successful reform is sometimes achieved only after decades of unsuccessful attempts. 


\subsection{Expanding abortion access: country examples}

Although the laws regulating abortion in Sub-Saharan Africa are generally restrictive, there are notable examples of progress toward expanding access to safe abortion. Several countries interpret their laws expansively, or have issued comprehensive safe abortion guidelines, to safeguard women's health. Below, we describe how three countries have enhanced the safety of abortion provision.

\section{Ethiopia-Category 3 \\ (plus all three most common additional grounds)}

Ethiopia stands out for having reformed its penal code to enable women to obtain legal abortion on the basis of their own assertion that their pregnancy has resulted from rape or incest. Further, its Technical and Procedural Guidelines for Safe Abortion Services (first issued in $2006^{63}$ and updated in $2013^{64}$ ) set evidence-based procedure and counseling guidelines for abortion and postabortion care. These guidelines mandate that to prevent repeat abortion, contraceptive services and counseling must be provided as an essential component of both types of care.

From the provider side, Ethiopia's guidelines authorize nurses and midwives to be trained in and provide first-trimester abortions using vacuum aspiration and the combination medication protocol (mifepristone plus misoprostol). As a result, the proportion of procedures done by such midlevel practitioners rose considerably between 2008 and 2014 , from $48 \%$ to $83 \% .^{65}$ The guidelines further specify that minors who qualify for an abortion by virtue of their age do not have to present proof of age.

As a result of the Ministry of Health's firm commitment to improving access to all medical services, including abortion, the number of public health centers increased by $250 \%$ between 2008 and $2014{ }^{66}$ The overall impact of these reforms - a trend toward safer procedures - likely contributed to the decline in obstetric admissions for complications of unsafe abortions, which fell from $47 \%$ of all such admissions in 2008 to 39\% by 2014 .

\section{Ghana-Category 4}

\section{(plus all three most common additional grounds)}

Ghana's expansion of legal grounds occurred relatively early, with a 1985 amendment to the penal code. ${ }^{67}$ The third edition of its Comprehensive Abortion Care Services Standards and Protocols (from 2012) meaningfully expanded access to safe services by not requiring a psychiatric assessment for women who would jeopardize their mental health by continuing their pregnancy. ${ }^{48}$ These guidelines also assert that providers cannot impose their beliefs and values on clients. The guidelines reinforce the notion of "comprehensive" abortion care as encompassing all aspects of safe abortion and mandate compassionate counseling for women receiving both legal abortions and postabortion care. ${ }^{11}$

Ghana's updated guidelines also prioritize use of the more effective combination medication regimen over misoprostol alone. Nationally representative data confirm that as of 2017, one-fifth of recent abortions were carried out through the combination regimen. ${ }^{54}$ Recent data from Komfo Anokye Teaching Hospital hint that improvements in safety are affecting the severity of treated complications, as the proportion of postabortion care admissions for septic abortions fell by $80-90 \%$ between $2010^{68}$ and $2019 .^{69}$

\section{Zambia-Category 5}

(with the additional legal ground of grave fetal anomaly)

Zambia is the only Sub-Saharan country in Category 5, meaning that abortion is legally permitted on all survival, health and socioeconomic grounds. While this has been the law since $1972,{ }^{15}$ it has tended to be a "paper law" rather than one that ensures widespread access. The continued high prevalence of unsafe abortion despite such a liberal law ${ }^{70}$ shows how hard it is to overcome the stigma that prevents women from openly seeking — or even talking aboutlegal abortions..$^{52,59,71}$ The country's 2017 Standards and Guidelines for Comprehensive Abortion Care contains many positive elements. ${ }^{58}$ After acknowledging the scarcity of high-level medical personnel, the guidelines extend authorization to provide abortions to trained midlevel practitioners. The guidelines are unusual in asserting that women must be informed about the range of abortion methods to choose from and that counseling must be empathetic, nonjudgmental and respectful.

Zambian providers cannot claim conscientious objection in an emergency. Should they do so under normal circumstances, they must "respectfully" refer the woman to a willing provider. Further, the guidelines mandate that precautions be taken to ensure women's confidentiality and that the best interests of a minor take precedence over the interests of her parents or guardian. However, women cannot benefit from these standards if they remain unaware of when they qualify for an abortion: Four decades after reform, only a small minority of women know the specifics of Zambia's abortion law. ${ }^{51}$ 


\section{Abortion Incidence and Trends}

A ccurately measuring the incidence of induced abortion poses serious challenges (as can be the case with any deliberately hidden behavior). Over the past decade, researchers have developed several methodologies to improve the estimation of abortion incidence at the country and city levels. Moreover, recent statistical modeling approaches have yielded updated global, regional and subregional estimates (see Data and Methods Appendix, page 39 ). ${ }^{6}$ This chapter summarizes the most up-todate information available on abortion incidence in Sub-Saharan Africa produced by these efforts.

\section{Current abortion incidence and trends}

As of 2015-2019, an estimated eight million abortions occur each year in Sub-Saharan Africa.? Because Eastern and Western Africa contain the region's two most populous countries (Ethiopia and Nigeria), these two subregions account for the highest numbers of annual abortions- 3.5 million and 2.7 million, respectively (Appendix Table 2, page 44). The total translates to an annual rate for SubSaharan Africa of 33 abortions per 1,000 women aged 15-49, a rate far lower than that seen in Asia (45), roughly the same as that in Latin America and the Caribbean (32), and much higher than the rates in Europe (20) and Northern America (12). ${ }^{72}$

The world's annual abortion rate remained basically unchanged between 1990-1994 and 2015-2019 when it was estimated at 39-40 per 1,000 women aged $15-49 .^{7}$ The same is true for Sub-Saharan Africa over this broad time frame: Despite an initial increase between 1990-1994 and 1995-1999, the regional abortion rate has remained virtually the same over the past 25 years (at 31-33 abortions per 1,000 women; Figure 3.1, page 18, and the Supplementary Table online at https://www.guttmacher.org/report/ from-unsafe-to-safe-abortion-in-subsaharan-africa).

But this apparent stability in rates does not necessarily mean stability in numbers. Because of persistent high fertility in several Sub-Saharan countries, ${ }^{73}$ combined with decreased child mortality, ${ }^{74}$ this region has the highest rate of population growth. When abortion rates remain stable but the population size increases, the absolute number of abortions will also increase. In fact, the absolute number of abortions per year in Sub-Saharan Africa almost doubled from 1995-1999 to 2015-2019, from 4.3 million to 8.0 million. ${ }^{7}$ The subregion with the smallest increase in the number of abortions was Southern Africa, the area with the lowest rate of population growth. ${ }^{75}$

Overall, abortion rates in Sub-Saharan Africa vary little by subregion (30-35 per 1,000 women). Middle and Southern Africa share virtually the same abortion rate (30-32 per 1,000), even though abortion is legal without restriction as to reason for $88 \%$ of women aged 15-49 in Southern Africa and for fewer than $1 \%$ of those in Middle Africa. (In Middle Africa, abortion is broadly legal only in Sao Tome and Principe, ${ }^{18}$ where just 52,000 women of reproductive age live. ${ }^{24}$ ) This finding affirms the worldwide finding that abortion incidence is by and large unrelated to legality.

Nonetheless, subregional rates mask wide variations in incidence at the country level. Studies using the same indirect estimation methodology, the abortion incidence complications method (AICM), were conducted between 2008 and 2016 in seven countries in Eastern Africa and three countries in Western Africa. The most recent AICM data show annual abortion rates ranging from lows of 16-17 abortions per 1,000 women in Senegal and Zimbabwe to a high of 48 per 1,000 in Kenya., ${ }^{6,76}$ In between are Burkina Faso, Ethiopia and Rwanda, with rates in the 20s, and Malawi, Nigeria, Tanzania and Uganda, with rates in the $30 \mathrm{~s}^{6}{ }^{6}$ Noncomparable data for Ghana from a different methodology combining several approaches found a rate of 44 abortions per 1,000 women of reproductive age in $2017 .^{77}$

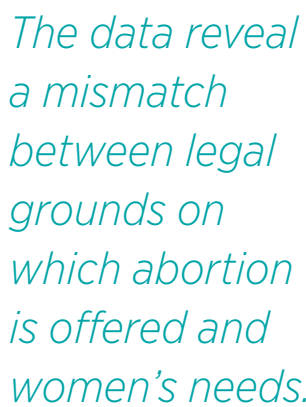

women's needs. 


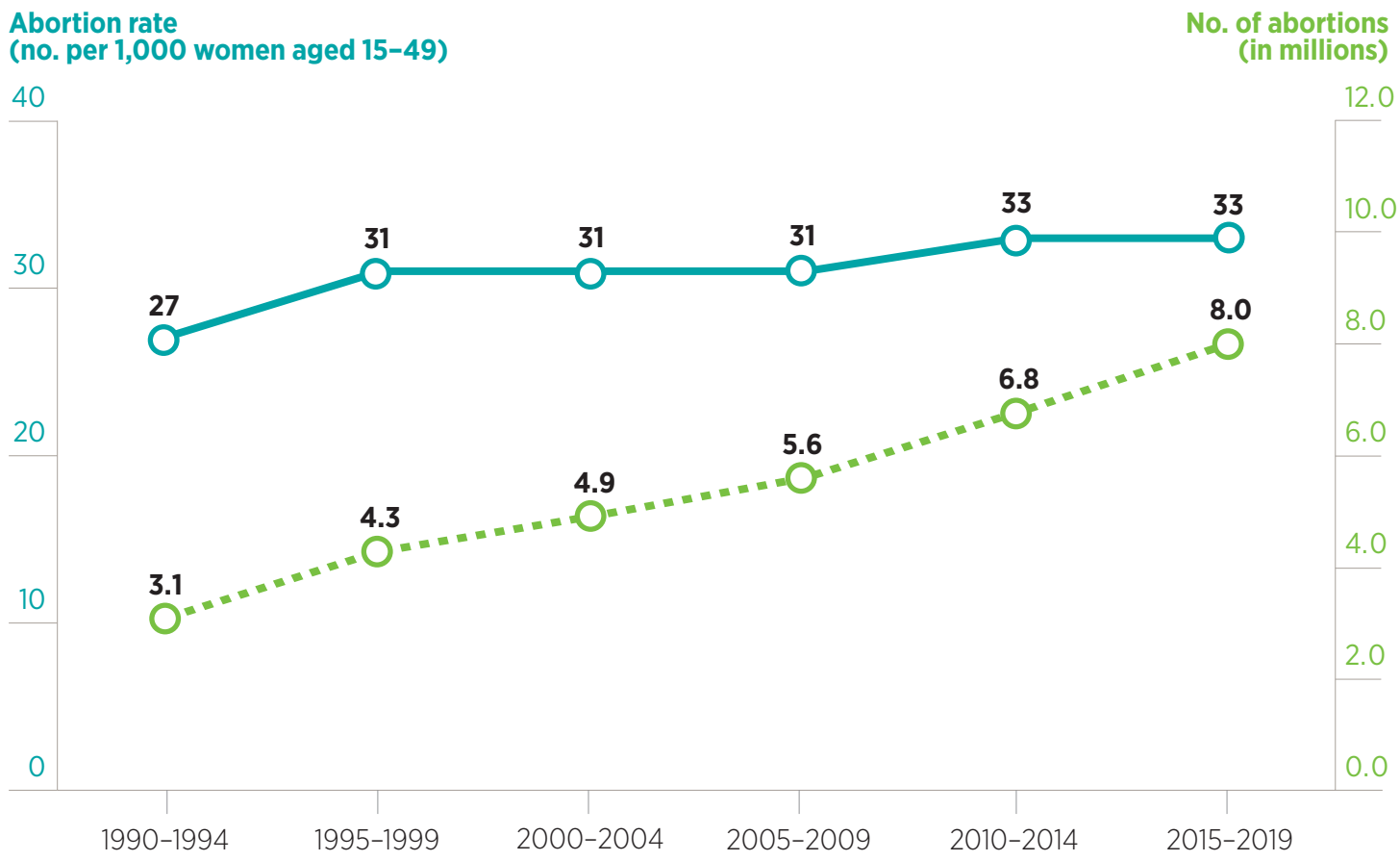

Source: reference 7 .

\section{Abortion incidence by characteristics}

Because childbearing before marriage is strongly stigmatized throughout Sub-Saharan Africa, many unmarried women who become pregnant likely want to avoid carrying an unintended pregnancy to term. Since most women in the region eventually marry (those in Southern Africa being the notable exception ${ }^{78}$ ), the bulk of unmarried women tend to be young. Abortion rates usually are lowest among the women at both extremes of reproductive age (i.e., among 15-19-year-olds and 45-49-year-olds) and peak among women in their mid- to late-20s. ${ }^{6}$ However, because many adolescent women (defined throughout this analysis as those aged 15-19) have not yet had sex, uncovering the true level of abortion among these youngest women requires limiting the data to only those who are at risk of pregnancy-sexually active adolescents (defined as those who have had sex in the past year).

Sexually active adolescent women in Sub-Saharan Africa have abortions at far higher rates than do all women of reproductive age. These rates, which are available for only a handful of countries, range from 75-76 abortions per 1,000 in Burkina Faso ${ }^{79}$ and
Uganda ${ }^{80}$ to 123 per 1,000 in Kenya. ${ }^{81}$ To understand the extent to which basing abortion rates on all adolescents underestimates the true level of abortion in that age-group, removing adolescents who are not yet sexually active increases the abortion rate nearly threefold in Uganda (from 28 to 76 per 1,000$)^{80}$ and almost fivefold in Ethiopia (from 20 to 91 per 1,000; Box 3.1, page 19). ${ }^{82}$

The preceding abortion data cover sexually active adolescents in these countries, no matter where they live. Yet urban or rural residence can be associated with many of the important factors that influence levels of unintended pregnancy and the decisions women of all ages make about continuing or ending such pregnancies. Although urban women's higher modern contraceptive use means they have lower unintended pregnancy rates than rural women, urban women may be far more motivated than rural women to resolve an unintended pregnancy through abortion, ${ }^{113}$ and abortion services and providers tend to be concentrated in urban areas. Further, highly educated and wealthier women often settle in urban areas; these women tend to want fewer children and have a smaller ideal family size, ${ }^{114}$ and they may have more 
resources to act on these preferences. In addition, urban women usually have higher levels of agency in reproductive decision making; such higher levels of empowerment are also associated with desires for fewer children. ${ }^{115}$

Abortion rates in large cities are indeed higher than comparable national rates (Figure 3.2, page 20). For example, in Ethiopia, the national rate is 28 and the rate in its largest city and capital, Addis Ababa, is $92 .{ }^{116}$ The difference is far smaller in Senegal, where the national rate is 16 , compared with a rate of 20 in Dakar. ${ }^{117}$ For the Democratic Republic of the Congo, data are available only for its capital city, Kinshasa; that city's rate of 56 (not shown) falls roughly at the midpoint for the five countries for which data are available for their largest cities. ${ }^{118}$

\section{Who has an abortion and why}

The limited information available from women who disclose having had an abortion or who seek care for abortion complications (as opposed to those seeking postabortion care for a miscarriage) shows that Sub-Saharan Africa follows a general pattern: Women seeking abortions are mostly young, unmarried, still in school, not yet a mother, living in an urban area and-compared with the general population of women-better educated and wealthier. For example, in Northern Ghana, the proportion of women who had an abortion is twice as high in urban as in rural areas. ${ }^{119}$ Similarly, a national-level study in Côte d'Ivoire in 2007 found that wealthier women and urban residents sought abortions more frequently than poorer women and rural residents. ${ }^{120}$

\subsection{Sub-Saharan adolescents and abortion}

Throughout the subcontinent, the gap between the age at which women initiate sex and the age at which they marry is growing. ${ }^{83}$ During this period of premarital sexual activity, adolescents may be exposed to heightened risk of unintended pregnancy. Among adolescents who become pregnant when they do not want to be ( $46 \%$ of all pregnancies among adolescents in Sub-Saharan Africa are unintended ${ }^{84}$ ), many seek abortion—usually clandestinely, because of the region's predominantly restrictive legal settings. ${ }^{20}$ Some $46 \%$ of unintended pregnancies among adolescents end in abortion, ${ }^{84}$ even though $90 \%$ of Sub-Saharan countries have restrictive abortion laws.

National laws (e.g., ministerial decrees, and penal and child codes) in eight Sub-Saharan countries-Benin, Central African Republic, Eritrea, Ethiopia, Gabon, Guinea, Mozambique and Rwandapermit minors to obtain an abortion (Table 2.1)..$^{22,23,85-90}$ Minor status as a ground for legal abortion is barely mentioned in the academic literature on abortion, and it is likely not widely known and rarely implemented. Very few countries have data on abortion rates among only those adolescents who are at risk for pregnancy. These limited data show that sexually active adolescents have the highest abortion rates of all women of reproductive age. ${ }^{79-82}$

The very large majority of unintended pregnancies among SubSaharan adolescents (86\%) occur among the 6.5 million who have an unmet need for modern contraception. ${ }^{84}$ These are the women who are exposed to the risk of pregnancy (because they are married, or are unmarried and sexually active) and do not want to have a child in the next two years, but are not using any contraceptive method or are using a traditional method. When sexually active adolescents are considered separately by marital status, levels of unmet need for modern methods are nearly always higher among those who are unmarried..$^{91}$ In countries where traditional method use is relatively common (i.e., at a level of at least $5 \%$ ), use is far higher among sexually active unmarried adolescents than among married adolescents.

Modern contraception is beyond many adolescents' financial reach, and some hesitate to use contraceptive services out of fear that providers will judge them for having sex before marriage. ${ }^{92,93}$ Indeed, some adolescents cite not being married as a reason for their not practicing contraception; many also mention concerns about side effects, infrequent sex and others' opposition to their using contraceptives. ${ }^{94}$

Abortion generally incurs far higher social and health costs for adolescents than for older women. For example, because of strong stigma against abortion, which can damage marriage prospects, ${ }^{95,96}$ many adolescents feel compelled to seek out a clandestine and potentially unsafe abortion. ${ }^{97-100}$ Adolescents generally recognize pregnancy later than older women, take longer to decide on an abortion and need more time to get the money to pay for one. ${ }^{101,102}$ All of these factors may push adolescents' abortions later into pregnancy, which increases the likelihood (relative to that of older women) that they will need postabortion care. ${ }^{103-107}$ Adolescents' treated abortion complications tend to be more severe than those of older women, and the reasons that lead adolescents to delay having an abortion-i.e., their reluctance to risk being recognized-also contribute to delays in seeking lifesaving postabortion care. ${ }^{107-112}$ 
Burkina Faso, 2008

Ouagadougou

Ethiopia, 2014

Addis Ababa

Rwanda, 2009

Kigali

Senegal, 2012

Dakar

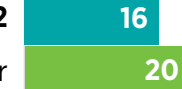

Uganda, 2013

Kampala
25

28

28
23

\section{3}

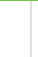

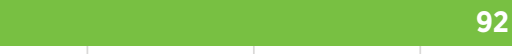

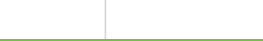

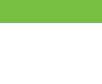

20
80

Country

Largest city
39

77

80

100

Annual abortion rate*

*No. of abortions per 1,000 women aged 15-49. Sources: references 79, 116, 117, 224 and Prada E et al., Incidence of induced abortion in Uganda, 2013 : new estimates since 2003, PLOS ONE, 2016, 11(11):e0165812, doi:10.1371/journal.pone.0165812.

Women have varied-and often multiple-reasons for interrupting a pregnancy. A 2016 study of postabortion care patients in Kinshasa found that the top two reasons women gave for having had an abortion were related to avoiding stigma: Fortytwo percent mentioned that they sought an abortion because they were unmarried (affirming the belief that abortion is common among unmarried women, whose marriage prospects may be hurt if word gets out ${ }^{95-97,121}$, and $23 \%$ said they wanted to protect family honor. ${ }^{122}$ Financial pressures were mentioned next most often. Being unmarried and wanting to continue studying were the most common reasons for abortion given by women who were treated after an unsafe abortion at three hospitals in Chad in 2015-2016. ${ }^{123}$

Although information is lacking on reasons for abortion by women's age, many reasons listed above reflect where women are in their life cycle. According to nationally representative surveys in Gabon, Ghana and the Republic of Congo, many women's main reason for abortion was related to their being young: not being ready to become a mother or wanting to delay it, wanting to continue schooling and fearing parents' negative reaction. ${ }^{124}$ Feeling unready or too young to start a family was a frequently reported reason in small-scale studies of women presenting for treatment of complications in Ghana. ${ }^{125,126}$ Relatively few women said that the main reason they turned to abortion was to limit their family size-a reason cited by just $3-5 \%$ of women in Gabon, ${ }^{127}$ Ghana ${ }^{128}$ and the Republic of Congo. ${ }^{129}$ This finding is consistent with the assumption that older women's desires to avoid higher parity births are not strong enough to motivate them to seek abortion.

Nationally representative trend data from Ghana, where abortion is broadly accessible despite a moderately restrictive law (Box 2.1, page 16), show that about one-third of women in both 2007 and 2017 sought an abortion for reasons mentioned above related to just starting out in life. ${ }^{54,55}$ Notably, the data reveal a mismatch between legal grounds on which abortion is offered ${ }^{67}$ and women's needs: Just $4-7 \%$ of Ghanaian women said that they had had an abortion because their physical or mental health was threatened or because the fetus they were carrying had a severe anomaly. 


\section{Abortion Practice and Consequences of Unsafe Abortion}

egal abortions conforming to recommended _clinical standards rarely result in incomplete abortion or medical complications. ${ }^{130,131}$ Furthermore, a first-trimester abortion induced by a trained provider with a recommended method is far safer than a pregnancy taken to term: According to the only available data, the risk of dying of complications from a first-trimester legal abortion in the United States is just 7\% that associated with childbirth. ${ }^{132}$ Yet in Sub-Saharan Africa, many abortions still lead to preventable health consequences for women. This situation calls for urgent action, since the path to avoiding such preventable outcomes is clear.

Among the factors that determine the level of harm posed by unsafe abortion is whether a woman seeks out an untrained provider (or induces abortion on her own) and the risk posed by the method or methods used. Both are closely linked to desire for secrecy, cost and availability. ${ }^{5}$

\section{Abortion practice throughout the region} As is true for women around the globe, women in Sub-Saharan Africa resort to an extensive array of safe and unsafe methods of abortion; these can be effective methods meeting the strictest international standards or they can be folk methods, which often do nothing but harm. It should be possible to describe the methods used for abortion in at least the few countries that allow abortion on broad legal grounds or that interpret their law expansively. However, this is not the case, as even countries where abortion is legal rarely publish such data. According to study data from Ethiopia and Zambia, roughly two-thirds are done using the combination medication protocol and one-third using manual vacuum aspiration (MVA). ${ }^{133,134}$ South Africa, the only sizable country in Sub-Saharan Africa that allows abortion without restriction as to reason, collects data only on the numbers of abortions that occur, not on the specific methods used. ${ }^{135}$
Even less is known about the methods that women use clandestinely. Much of the available information on the methods that women use comes from postabortion care records, which are themselves often patchy and of low quality. (Such unclear recordkeeping can be deliberate, to shield women from prosecution.) By definition, postabortion care records do not reflect women who do not need care or those who need care and do not obtain it, including those who die before reaching a source of care. ${ }^{33}$

Women themselves often do not know what medicines or concoctions they took or what abortion providers did and can only vaguely describe the process. ${ }^{136}$ Because inexpensive, readily available methods often fail, many women end up making more than one attempt-starting with home remedies, out of a desire for privacy, and eventually seeking facility-based care when those initial methods fail. ${ }^{102,136}$ Indeed, many studies refer to the "final method used" to denote the method that actually worked. Because seeking out providers and waiting to see if a method worked takes time, making multiple attempts may push abortions later into pregnancy, when procedures are riskier, when fewer trained professionals are available to perform them and after some legal gestational limits have expired. ${ }^{137}$

The extent to which male partners are involved in these often difficult decisions about what method to use is barely known. Studies on male involvement-a factor that varies widely by the nature of the romantic relationship - use such different measures that results cannot reliably be summarized. ${ }^{138,139}$ We know that men often provide financial support, which may help women afford a safer abortion. ${ }^{140,141}$ Positive emotional support also means that the household decision to seek timely and appropriate treatment of complications from unsafe abortion is likely to be made quickly. ${ }^{141}$ Yet men are not always involved in or supportive of

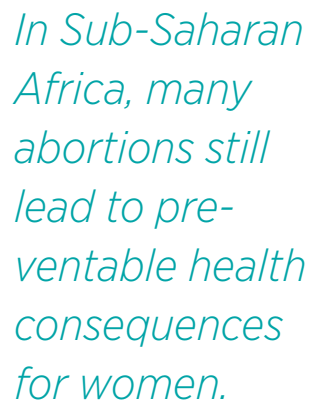




\subsection{Traditional abortion methods used in Sub-Saharan Africa}

\section{SUBSTANCES INGESTED/INSERTED}

Plants/plant-based infusions

- Aloe vera ${ }^{142} /$ burnt bean ashes ${ }^{142} /$ cassava leaves ${ }^{136} /$ garlic $^{136} /$ gourd $^{142} /$ honey $^{123} /$ lime tree $\operatorname{root}^{136} /$ mango tree seeds $s^{136} /$ plectranthus (spurflower) $^{136} /$ papaya-leaf poultice $e^{121} /$ boiled henna $\operatorname{root}^{136} /$ sisal leaves $^{143}$

Store-bought nonpharmaceutical items, usually consumed in large quantities

- Ammonia-based cleaning products (Handy Andy, Jeyes Fluid $)^{136,144} /$ baking soda $^{142} /$ beer $^{102} /$ blood tonics ${ }^{68} /$ brandy $^{144} /$ chalk $^{136} /$ Coca-Cola ${ }^{102}$ / Maggi cube (concentrated bouillon) ${ }^{145} /$ fish poison $^{143} /$ Nescafé $^{102,146} /$ steel wool mixed with Oro-Crush (soft-drink syrup) ${ }^{144} /$ toothpaste $^{142}$

Combinations of store-bought and plant-based substances

- Bark steeped in alcohol ${ }^{68} /$ lemon juice on a vaginal suppository ${ }^{143} /$ plants soaked in alcohol $^{68}$ / strong black tea plus chloroquine (antimalarial) $^{136}$

their partner's abortion. Factors that typically militate against men's involvement or support include their denial of responsibility for the pregnancy, suspicion of infidelity, or the woman's decision to not inform her partner about the pregnancy or the abortion decision, for fear that he would not support such a decision. ${ }^{139}$

Once a decision is made, a woman often proceeds with the abortion in secret, telling as few people as possible. Avoiding formal channels by using folk and traditional remedies can ensure privacy. These methods encompass a range of longstanding folk remedies mixed with newer, commercial products and substances (Box 4.1). Overall, ineffective abortion methods can roughly be organized into five categories: caustic substances, herbal remedies, pharmaceuticals or products erroneously believed to be abortifacients or to work as abortifacients if taken at high doses, sharp objects and intense physical exertion.

abortion" to refer to both the combined and misoprostol-only regimens. Although the term "medical" is often used, its multiple meanings in medicine can create confusion, and the term was also used to denote "safe" before the advent of medication abortion, as in the Englishlanguage criteria of the Maputo Protocol to "authorize medical abortion."
According to data from a 2007 nationwide household survey in Côte d'Ivoire, women most commonly reported having used herbal remedies (50\%) to terminate a pregnancy; $39 \%$ underwent dilation and curettage (D\&C) and $11 \%$ received injections of unknown substances. ${ }^{120}$ Unrelated

\section{PHARMACEUTICALS}

Over-the-counter, usually in large quantities

- Aspirin $^{142} /$ Cafemol (caffeine plus paracetamol) $)^{59} /$ folic acid ${ }^{145}$ / laxatives (castor oil and Epsom

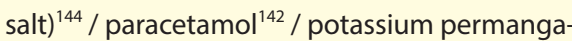
nate (wound cleaner) ${ }^{123} /$ snake antidote ${ }^{142}$

Antibiotics ${ }^{142,143}$

Antimalarials ${ }^{123,143}$

Deworming agents ${ }^{121,145}$

Vasodilators ${ }^{145}$

Uterotonics $^{68}$

Hormonal contraception ${ }^{68,102}$

\section{OBJECTS INSERTED INTO CERVIX}

Cassava sticks $^{143} /$ metal rods or wires ${ }^{142} /$ scissors ${ }^{142} /$ tree roots $^{142}$ nationwide research done in Côte d'Ivoire in 2018 showed that about $42 \%$ of women who had had an abortion used traditional remedies; misoprostol, which was just starting to be used at that time, was mentioned by only $4 \%$ of women. ${ }^{147}$ In Ghana, the proportion of abortions induced using misoprostol more than tripled from 2007 to 2017 (from 5\% to 18\%). In 2017, another one-fifth were induced by the combination protocol, which had not been available in the country long enough to be covered in the 2007 questionnaire. ${ }^{54,55}$ The more recent Ghanaian survey also shows progress away from the use of D\&C, as the proportion of women who had had such an abortion dropped from $40 \%$ to $24 \%$ over the decade.

\section{Medication abortion-most often misoprostol alone}

The use of medication abortion has transformed the landscape of reproductive health the world over. Of the method's many advantages, perhaps the most important for Sub-Saharan Africa is that it is far safer than the still-used (but no longer recommended) invasive surgical technique of D\&C. The combination protocol of mifepristone (which stops the pregnancy) followed by misoprostol (which causes uterine contractions) is significantly more effective than misoprostol alone. ${ }^{.148}$ The 
World Health Organization (WHO) recommends the combination protocol and advises that misoprostol alone be used when mifepristone is unavailable. ${ }^{149}$ Since mifepristone is far more expensive than misoprostol and has one major indication (to end a pregnancy) whereas misoprostol has several, it is generally unavailable throughout the areas of SubSaharan Africa with highly or moderately restrictive abortion laws. ${ }^{150}$ As a result, in this region, "medication abortion" in effect overwhelmingly means use of misoprostol alone.

Misoprostol was originally marketed as an ulcer medication (and is an active ingredient in arthritis drugs that some women use as abortifacients ${ }^{151}$ ); it is also used for treating both incomplete abortion ${ }^{152}$ and postpartum hemorrhage. ${ }^{153}$ In all likelihood, misoprostol first became available in the region sometime around 2005 , when it was added to WHO's list of essential medicines, a source many Sub-Saharan countries rely on when drawing up their national essential medicines list. ${ }^{154}$ The introduction of misoprostol as an abortion method may coincide with an initial spike in women unnecessarily seeking medical care at facilities, both because correct use of the method causes heavy bleeding, which women may not expect and may find alarming, ${ }^{6}$ and because pharmacists and other providers may tell women to seek care at a facility as soon as bleeding starts. This was often the case in Latin America, the first region where misoprostol alone was widely used to induce abortion. ${ }^{155}$

The clandestine nature of misoprostol use means that unscrupulous actors can sell adulterated, expired or otherwise ineffective pills. Samples of the drug from 15 countries, two of them in Sub-Saharan Africa (Kenya and Nigeria), revealed that $40 \%$ had less than the labeled content of misoprostol. ${ }^{156}$ Similarly, a recent analysis testing the composition of tablets from a range of outlets throughout Nigeria found that one-third of misoprostol pills did not contain enough of the drug's active ingredient to work. ${ }^{157}$ Moreover, the pills normally are protected by foil blister packaging; should the packaging seal be broken, exposure to heat and humidity quickly degrades misoprostol's potency, decreasing the method's effectiveness and thus its safety. ${ }^{158}$

Use of misoprostol clearly is becoming more common, with the drug now widely available in cities in several countries, including Ghana, ${ }^{159}$ Nigeria $^{160}$ and Tanzania. ${ }^{161}$ However, nationally representative information on where women get misoprostol and on how or whether they learn to use it correctly is scarce. The conventional wisdom is that womenor their partners, friends or family memberspurchase misoprostol without a prescription from a pharmacy or informal source. Pharmacies' accessibility and affordability make them the preferred first source for medication, and often for health care overall. ${ }^{162}$

Results of research on the use of misoprostol vary broadly, implying that countries are likely at different stages in the drug's introduction: In Kenya in 2013, about one-quarter of pharmacies visited offered to sell misoprostol to mystery clients, ${ }^{\mathrm{h}}$ and $3 \%$ offered them both mifepristone and misoprostol. ${ }^{163}$ In Zambia in 2009, 46\% of pharmacy workers offered women information about where they could obtain misoprostol or actually sold it to them, whereas just two years later $66 \%$ did so. ${ }^{164}$ Although such an increase is notable, information is lacking on the quality of the instructions that women received. The experience of Latin America suggests that it can take years for information on misoprostol's optimal timing, dosage and route of administration to spread. ${ }^{155}$ Until then, women who lack accurate instructions may end up unnecessarily risking incomplete abortion or medical complications from using a usually safe and effective method. ${ }^{3}$

For many — but not all_-methods of abortion, providers either supply women with the means to abort or carry out the abortion. However, data on the methods used by each provider type are lacking. We have data from nine countries on health professionals' estimates of the distribution of methods used. Moreover, misoprostol use was not yet widespread in most of the study countries at the time of data collection. As of the period 2009-2016, health professionals estimated that in eight of the nine countries with data, more abortions involved nonclinicians (i.e., traditional providers, pharmacists and the woman herself) than midlevel practitioners (nurses and midwives) or physicians. For example, the proportion of abortions involving nonclinicians ranges from $41-45 \%$ in Kenya, Nigeria and Uganda to $72-74 \%$ in Burkina Faso and Malawi (Figure 4.1, page 24). ${ }^{165}$

\section{The continuum of safety}

During the mid-2010s, WHO responded to the growing use of misoprostol for clandestine abortion by shifting their classification of abortion safety from binary categories (safe and unsafe) to more of a continuum. ${ }^{166,167}$ The revised classification h) In legally restrictive settings, misoprostol sales can often be studied only through the use of "mystery clients," as women who pose as clients are called. 


\section{1 In six of nine countries with national-level data, the majority of abortions are estimated to be carried out by a provider who is not a clinician.}

\section{$\%$ of abortions by type of provider}

100

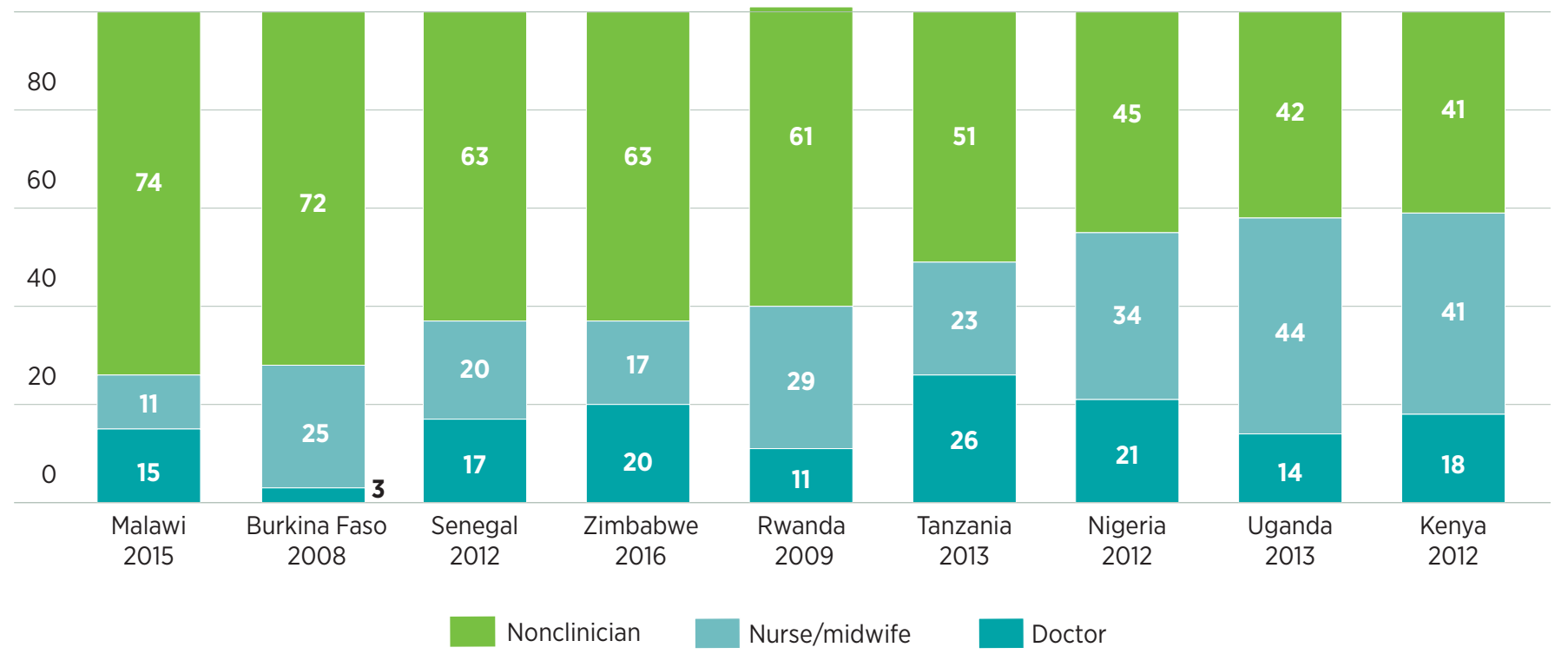

Note: Nonclinicians are pharmacists, traditional providers and women themselves. Clinicians are nurses, midwives and doctors. Source: reference 165

i) The Eastern African nation of Seychelles is high-income, but it accounts for less than $0.001 \%$ of women of reproductive age in the region. ${ }^{24}$ system divides abortions into three mutually exclusive gradations: Safe procedures are those that use a WHO-recommended method appropriate to the pregnancy duration and are done by a trained provider; less-safe abortions meet just one of these criteria; and least-safe abortions meet neither criterion. $^{5}$

According to WHO, as of 2010-2014, about three-quarters $(77 \%)$ of abortions in Sub-Saharan Africa are considered unsafe (the sum of less safe and least safe). ${ }^{2}$ Applying this proportion to the annual average of abortions estimated for 2015-20197 means that some 6.2 million women each year contend with the possible health consequences of unsafe abortions-consequences that would be avoided with safe procedures that adhere to WHO standards. Roughly half (49\%) of abortions in Sub-Saharan Africa qualify as least safe (Appendix Table 2, page 44). ${ }^{2}$ Other regions' levels of least-safe abortions are far lower, ranging from an undetectable level in Europe and Northern America to $8 \%$ in Asia and $17 \%$ in Latin America. ${ }^{5}$

Throughout most of the world, wealthier regions tend to have more liberal abortion laws, and thus abortions are safer there. For example, least-safe abortions account for a slight majority of abortions in low-income countries (54\%) but for fewer than $1 \%$ in high-income countries. ${ }^{5}$ However, the absence of a sizable high-income country in SubSaharan Africa prevents any testing for the usual association between national wealth and abortion law-and thus safety. By subregion, the prevalence of least-safe abortions is highest in Middle Africa, at $69 \%$ (Figure 4.2, page 25). At the other extreme is Southern Africa, where just $7 \%$ of abortions qualify as least safe. It is unsurprising that least-safe abortions are so uncommon in Southern Africa, given that South Africa, which decriminalized abortion in 1996, accounts for nearly $90 \%$ of the subregion's population.

\section{Treatment needed after abortion}

In Sub-Saharan Africa, as in other world regions, restrictive abortion laws do not prevent women from seeking abortion but can make their abortions unsafe, leading to the need for urgent care. Incomplete abortion is the most common reason for such care, ${ }^{3}$ and the incomplete abortion rate varies by the specific method used and when in pregnancy it is used. Women experiencing incomplete abortion risk developing severe, and even life-threatening, medical complications. 
Such medical complications require immediate attention, and much of the mortality associated with unsafe abortion can be traced to late ${ }^{168}$ (or deficient) postabortion care. In 2012, an estimated 1.3 million women received treatment following an induced abortion in Sub-Saharan Africa, for an annual rate of 6.7 treated cases per 1,000 women aged $15-44 .{ }^{169}$ Among the 10 countries in the region for which these rates could be estimated, annual treatment rates ranged from lows of 3-4 cases per 1,000 women in Ethiopia and Mauritius to highs of 12-13 per 1,000 in Kenya and Uganda.

According to indirect incidence studies conducted in 10 countries with restrictive abortion laws, some $44 \%$ of all abortions require facility-based care to complete the abortion or address medical complications (Figure 4.3, page 26). ${ }^{165}$ This proportion rises with women's relative disadvantage, going from $27 \%$ among nonpoor urban women to $52 \%$ among poor rural women. Overall, some $43 \%$ of women who need treatment do not get it, and this burden of mostly avoidable suffering falls disproportionately on the most disadvantaged women. Half of poor rural women do not receive needed care following an abortion, whereas just one-fifth of nonpoor urban women are in this situation of needing but not getting care.

Regarding the potentially safe and effective selfuse of misoprostol, many women do not get correct information on what to expect or how to use it. ${ }^{170}$ The number of women who present for postabortion care because of heavy bleeding when in fact they are experiencing the expected signs of a misoprostol abortion cannot be quantified. A study in Kinshasa, Democratic Republic of the Congo, found that $5 \%$ of women seeking postabortion care (after an abortion or miscarriage) did not require treatment; ${ }^{111}$ all of these women had used misoprostol and were discharged in good health in less than 24 hours. However, ongoing evidence collection on the severity of complications in the region implies that a trend toward more informed self-use of misoprostol has begun in a few countries.

\section{Severity of complications}

The severity of complications is closely related to the abortion method used and when in pregnancy the abortion occurs. Postabortion care records (for treatment following abortion or miscarriage ${ }^{j}$ ) show a substantial burden of severe medical complications from unsafely induced abortion:The proportion of complications estimated to be severe ${ }^{k}$
Southern Africa, where abortion is broadly legal, is the only subregion in which safe abortions are far more prevalent than unsafe abortions.

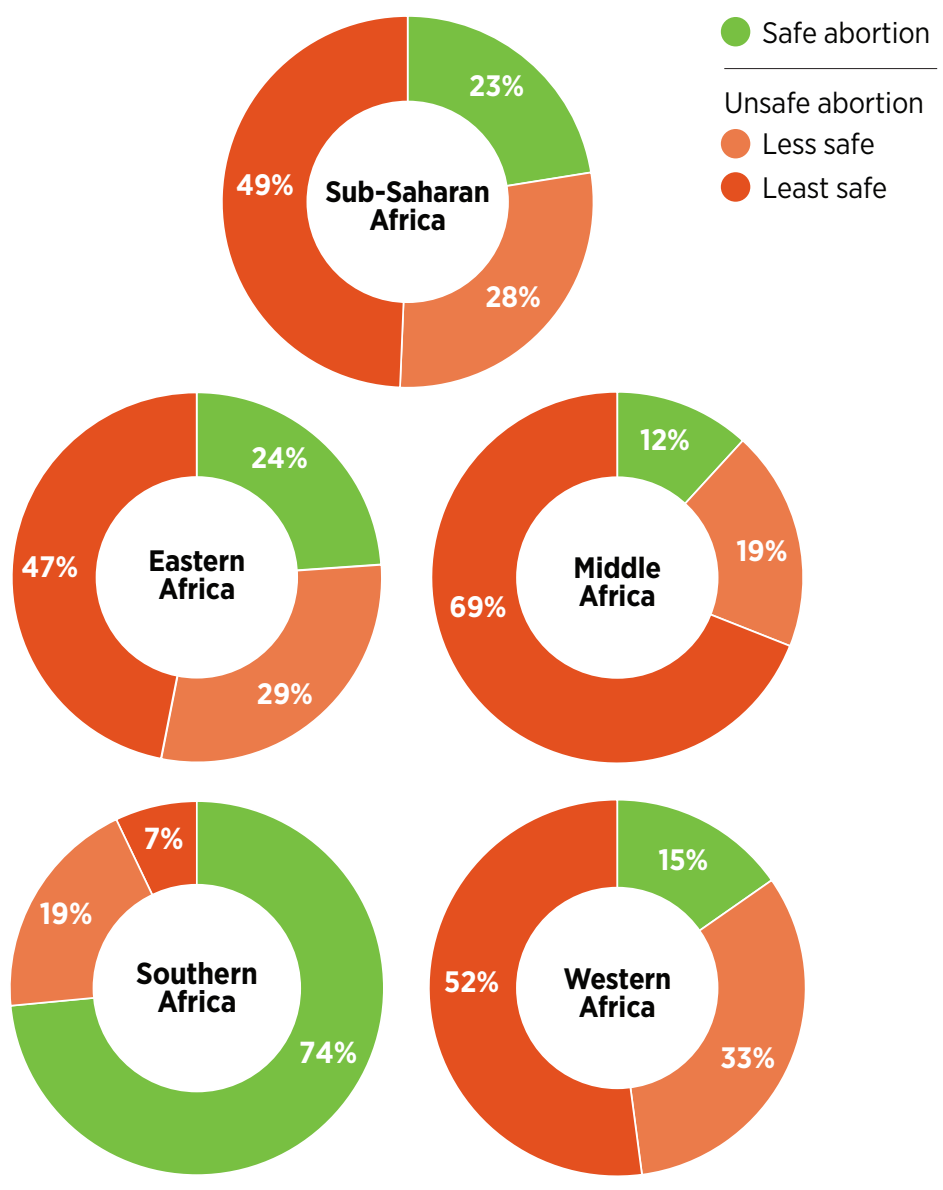

Note: Safe abortions are those that use a WHO-recommended method appropriate to the pregnancy duration and are done by a trained provider. Less-safe abortions meet only one of these criteria, and least-safe abortions meet neither criterion. Sources: references 2 and 5.

ranges from $20 \%$ in Uganda to $37 \%$ in Kenya. ${ }^{172}$ According to 2016 data from Kinshasa, among postabortion care patients who needed treatment (after the removal of the misoprostol patients who did not), $17 \%$ of abortion-related complications were severe. ${ }^{111}$ More-severe complications not only mean higher personal and health costs to women and their families, but also higher financial costs to national health systems: In Malawi in 2009, the cost of treating complications that were severe but did not require serious surgical repair was nearly five times the cost of treating a simple complication. ${ }^{173}$ Similarly, in Sierra Leone in 2011, the cost for treating severe complications averaged nearly eight times that of a simple case. ${ }^{174}$

Delays in getting care can worsen the severity of complications. Many women postpone postabortion care because they hope that the problem will j) Postabortion care refers to treatment needed after abortion or miscarriage. Because the symptoms of complicated miscarriages and complicated abortions are similar (especially in the case of medication abortion), women understandably seek to evade stigma or censure by reporting than an abortion. ${ }^{171}$ Although some studies differentiate between cases of abortion and miscarriage, many do not (or are unable to); thus, complicated miscarriages are often included with abortions in postabortion care data.

k) In Ethiopia, Kenya, Malawi and Zimbabwe, severe cases refer to the diagnosis of one or more of the following: pelvic abscess, uterine perforation, peritonitis, sepsis (and septic shock), need for surgery and an unknown cause that results in the woman's death In Uganda, severe complications refer to one or more of these last three, plus receipt of a blood transfusion. that they had a miscarriage rather 
resolve on its own. Other reasons for delaying postabortion care overlap with why many women put off getting an abortion in the first place: wanting to avoid being recognized or discriminated against, needing time to get the money together or arrange for transportation, and fearing arrest. ${ }^{103}$ That fear is well-founded, as most arrests of women for abortion are initiated by the medical personnel whom they go to for help; in addition, some providers demand bribes or even refuse to provide care. ${ }^{175-177}$ The fear of being shamed, and possibly arrested, also figures in decisions to seek care instead from traditional healers ${ }^{178}$ or to forgo it altogether. ${ }^{179}$

Treatment delays can also occur once a woman seeks care. Local health centers often lack trained staff or equipment, requiring time-consuming referrals to higher-level facilities. In Kenya in 2012, not getting care within the first six hours after the onset of symptoms was found to double the odds that complications would be moderate or severe (as opposed to mild). ${ }^{109}$ In Gabon, providers were shown to make women who had had an abortion wait longer for care than other women, potentially worsening their health outcomes. ${ }^{180}$

The timing in pregnancy of the abortion also contributes, as severe complications are significantly more likely to occur with second-trimester abortions than with earlier abortions. ${ }^{109}$ For example, 2016 data on complications of abortion and miscarriage in Kinshasa indicate that severe complications (as opposed to mild or moderate complications) were nearly four times as likely to occur among women whose pregnancy ended after the first trimester as among those whose pregnancy ended in the first trimester. ${ }^{111}$ The same pattern was seen in Malawi and Zimbabwe (though to a lesser degree), as women seeking postabortion care after the first trimester had $30 \%$ higher odds of experiencing increasingly severe complications, compared with those whose pregnancy ended in the first 12 weeks of gestation. ${ }^{108,110}$ As mentioned earlier, complicated miscarriages are included in these dataindeed, postabortion patients are broadly admitted as miscarriage patients in settings where abortion is not broadly legal ${ }^{181}$ — which means that the data underestimate the severity of complications from abortion alone.

\section{Mortality from abortion}

If treated too late or not at all, severe complications from unsafe abortions can lead to death. Many Sub-Saharan African studies present the percentage of women who died in health facilities from abortion-related complications. However, these values fluctuate widely, both over time and across countries. The annual abortion case-fatality rate,

\section{3 \\ Poor women in rural areas risk the most by having an abortion, as more than half of their abortions are estimated}

to need treatment.

$\%$ of abortions, according to whether they resulted in complications

100
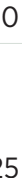

0

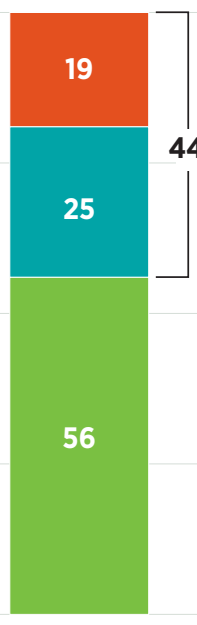

All women*

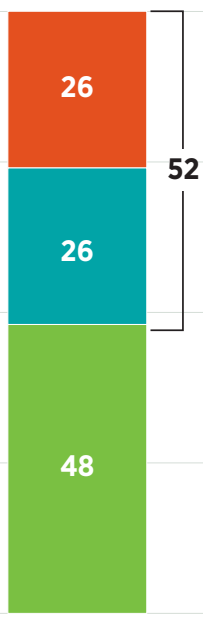

Poor rural

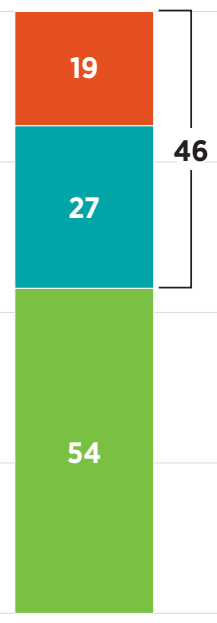

Poor urban

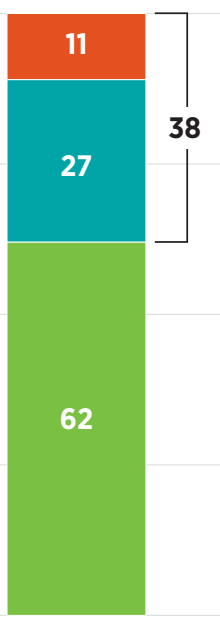

Nonpoor rural

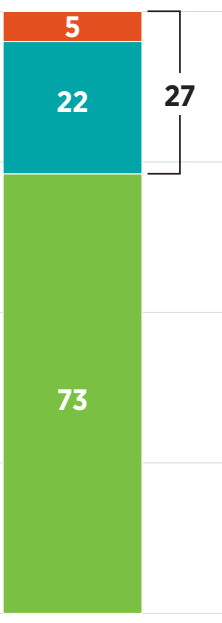

Nonpoor urban

*The distribution for all women is calculated as a weighted average of the four subgroups. Note: Data come from the following 10 countries: Burkina Faso, Ethiopia, Kenya, Malawi, Nigeria, Rwanda, Senegal, Tanzania, Uganda and Zimbabwe. Sources: reference 165 and special tabulations of data from the 2014 Health Professionals Survey for Ethiopia. 
or the number of maternal deaths per 100,000 abortions, is a more useful and comparable measure. This value also includes the deaths of women who never received treatment. (See the Data and Methods Appendix for how case-fatality rates were calculated.) As of 2019, Sub-Saharan Africa has the highest annual case-fatality rate of any world region, at roughly 185 maternal deaths per 100,000 abortions. ${ }^{8}$ Rates for other regions of primarily lowand middle-income countries, such as Asia and Latin America and the Caribbean, are just 14 and 16 deaths per 100,000 abortions, respectively.

Sub-Saharan Africa's rate translates to nearly 15,000 preventable and untimely maternal deaths each year. ${ }^{84}$ The subregion of Middle Africa has the highest case-fatality rate (about 260 deaths per 100,000 abortions), with Western Africa close behind (roughly 225 per 100,000). Fewer women die for every 100,000 abortions in Eastern Africa (about 160), and unsafe abortion leads to the lowest case-fatality rate in Southern Africa, the sole subregion where legal abortion predominates (roughly 30 deaths per 100,000 abortions each year). Trend data are encouraging: From 2000 to 2019 , as the quality and coverage of postabortion care improved, and as clandestine use of misoprostol likely increased, fewer women died from severe consequences of unsafe abortion. At the regional level, the case-fatality rate declined by about twofifths, from roughly 315 to 185 per 100,000 (see Supplementary Table online), which marks meaningful progress toward saving women's lives.

The percentage decline since 2000 in case-fatality rates was largest in the one subregion where a sizable country expanded access to legal abortions over the period: The rate fell by $53 \%$ in Eastern Africa, probably as a result of improvements in access to safe abortion services in Ethiopia, where more than one-quarter of the subregion's population lives. ${ }^{24} \mathrm{~A}$ study that compiled mortality data from several sources estimated that the proportion of maternal deaths that were linked to abortion in that country declined from $31 \%$ in $1980-1999$ to $10 \%$ in $2000-2012 .^{182}$

Quality and methods of postabortion care Treatment for incomplete abortion (which accounts for the large majority of postabortion care cases) involves a procedure similar to that used to provide legal abortion services. WHO's evidence-based clinical standards for treating incomplete first-trimester abortions ${ }^{3,131,183}$ recommend the use of vacuum aspiration' or misoprostol. Using misoprostol for postabortion care has multiple, interrelated advantages in low-resource settings such as Sub-Saharan Africa, including that the drug can be purchased cheaply and stored easily, no extensive training or equipment is required, plentiful midlevel personnel can administer misoprostol and it can be made available in primary care settings in remote rural areas. ${ }^{152}$ Indeed, results of interventions to introduce misoprostol for postabortion care $\mathrm{e}^{152,184,185}$ and of interviews with postabortion patients treated with misoprostol show high levels of satisfaction with this treatment method, which is perceived as safe and natural. ${ }^{186}$

However, the transition away from use of D\&C (an invasive surgical method not recommended by WHO) is far from complete. Use of D\&C to treat women needing care following an abortion or miscarriage was still fairly common, as of 2016, in Zimbabwe (63\% of such cases) ${ }^{108}$ and in Kinshasa (49\%). ${ }^{111}$ And as of 2011, this method was being used to treat the large majority (85\%) of women needing postabortion care in Sierra Leone. ${ }^{174}$

Several reasons may explain the predominance of $D \& C$ for postabortion care in some countries. Among these are inadequate supplies of misoprostol commodities and vacuum aspiration kits. Indeed, donated MVA equipment often wears out and is not replaced, and many facilities lack the ability to sterilize MVA equipment. ${ }^{12,187-189}$ Another likely reason for the ongoing use of $D \& C$ is that medical doctors, who were trained in D\&C and who disproportionately occupy senior positions, often resist having postabortion care procedures done by more junior personnel, who may have been trained in safer modalities. ${ }^{188}$

The case of Malawi demonstrates the danger of moving away from recommended practices. According to a small-scale study in three Malawian public hospitals, MVA was used in more than half of first-trimester postabortion care cases in 2010. After funding for training and equipment stopped, this proportion dropped to one-quarter in 2011 and to one-tenth in 2012. ${ }^{189}$ This drop in MVA use was made up for by increased reliance on D\&C, which was used for $90 \%$ of first-trimester postabortion care patients in 2012.

The ability to provide good-quality, comprehensive postabortion care depends not only on the availability of trained personnel, but also on having the medical supplies and equipment needed to carry out the essential components of postabortion

\section{If treated too late or not at all, severe complications from unsafe abortions can lead to death.}

I) Although vacuum aspiration can be manual or electric-they are equally safe and effectivewe refer primarily to the manual variant going forward because it is overwhelmingly what providers in the region are trained in and use. 
Treating abortion

complications

costs farmore than paying for a safe abortion to begin with. care, often referred to as "signal functions." Roughly one-quarter of referral hospitals in Uganda and Namibia and one-third in Kenya, Rwanda and Senegal had such requisite signal functions to provide comprehensive postabortion care in 2007-2015..$^{190}$ In Zimbabwe as of 2016, 100\% of tertiary-level facilities providing acute care in urban areas had stockouts of misoprostol-the most cost-efficient method for treating incomplete abortion-and $49 \%$ of all facilities at all levels lacked functional kits to perform MVA. ${ }^{76}$ Lack of equipment and commodities likely explains the use of forceps and fingers for uterine evacuation in Kinshasa (27\% of postabortion care cases), ${ }^{111}$ Kenya (19\%) ${ }^{109}$ and Zimbabwe (12\%). ${ }^{108}$

Discussing and providing contraception as part of postabortion care is an essential link in the chain of preventing future unintended pregnancy and unsafe abortion. Data from public hospitals in Ethiopia, Ghana, Nigeria, South Africa and Zambia over the period 2011-2013 document that from $42 \%$ (in South Africa) to $86 \%$ (in Ethiopia) of legal abortion and postabortion care clients adopted a contraceptive method. ${ }^{191}$ In Kenya, young women were significantly less likely than older women to leave their source of care with a contraceptive method. ${ }^{192}$ According to most studies of postabortion contraceptive care, including pilot projects, women commonly leave their source of postabortion care with the injectable. ${ }^{193}$ Indeed, this is the most frequently used method in the region, and its predominance is unique among developing regions. The predominance of the injectable suggests that women in Sub-Saharan Africa may not always be offered the full range of contraceptive methods. $^{194}$

\section{Impact of postabortion care costs}

Where abortion is legal, ensuring the availability and affordability of safe services is not only critical to women's health and well-being, it is also costeffective. As of 2019, an estimated $\$ 228$ million is spent in Sub-Saharan Africa each year on treatment for complications from unsafe abortion. ${ }^{84}$ Given the subregion-specific proportions of abortions that are unsafe and the relative sizes of the populations of reproductive-age women, Eastern Africa and Western Africa make up the bulk of this amount (\$88 million and \$87 million, respectively), followed by Middle Africa ( $\$ 43$ million) and Southern Africa (\$10 million). The magnitude of the costs for individual countries' health systems to treat abortion complications likely varies depending on the incidence of unsafe abortion, the population size and the protocols for maximizing the use of midlevel practitioners and for decentralizing care to primary-level facilities.

These national-level costs do not fully reflect the costs borne by individuals. The women who can afford only the least expensive-and thus often unsafe-abortions are the ones who end up having to shoulder the highest postabortion care costs in all legal settings. In broadly legal settings specifically, elevated (sometimes illegal ${ }^{59}$ ) charges for legal abortions perpetuate unsafe abortions, and thus the need to also pay for postabortion care. ${ }^{171}$ Apart from these direct costs of care, women pay in other ways: Among Ugandan women who had been treated for unsafe abortion, three-quarters mentioned a resulting loss in productivity and one-third a deterioration in their economic circumstances. ${ }^{195}$

Studies consistently show that treating abortion complications costs far more than paying for a safe abortion to begin with. In Sierra Leone, which bans abortion outright, health system costs to treat an unsafe abortion are twice as high as the costs of a safe procedure; ${ }^{174}$ similar research in Zambia shows that treating complications costs 2.5 times as much as providing a safe procedure. ${ }^{133}$ Moreover, in Burkina Faso, postabortion care costs at two referral hospitals are 7.3 times the cost of safe abortion services. ${ }^{196}$ Overall, the most cost-effective facility-based abortion method for a given country depends on a country's specific medical system costs and funding levels. The bottom line is that increasing access to abortion services that use any safe method will always save lives and costs relative to clandestine, unsafe abortions. ${ }^{197}$ 


\section{Unintended Pregnancy, the Underlying Cause of Abortion}

A bortions overwhelmingly involve pregnancies that start out as unintended. It is likely that only a very small proportion are interrupted for medical reasons or because of unanticipated changes in relationship or financial circumstances. To have just two or three children and maintain the medically recommended birthspacing of at least 33 months, ${ }^{\text {m198 }}$ women and couples would need to be protected from unintended pregnancy for most of their reproductive lives. ${ }^{199}$ Although wanted fertility is currently higher in Sub-Saharan Africa than in any other world region, ${ }^{200}$ it has started to decline in all subregions except Middle Africa, where four $^{\mathrm{n}}$ of the five countries with data show no real change in the measure over time. ${ }^{114}$ According to recent data from the two countries with the largest ideal family size, Chad and Niger, a generational shift is evident: In Chad, 45-49-year-old women consider just over nine children to be ideal, while 15-19-year-olds view slightly more than seven as ideal; ${ }^{201}$ in Niger, ideal family size among these two age-groups averages nearly 11 and just over eight, respectively. ${ }^{202}$

\section{Incidence of unintended pregnancy}

As might be expected given their preferences for large families, women in Sub-Saharan Africa have the highest pregnancy rate among all world regions: Each year, 218 of every 1,000 women in the region become pregnant (Appendix Table 2), compared with 129 per 1,000 in Asia, 110 in Latin America, 75 in Europe and 77 in Northern America. ${ }^{72}$ Because the region's pregnancy rate is so high, its unintended pregnancy rate is also very high, despite the region having the lowest proportion of pregnancies that are unintended: Some 91 unintended pregnancies occur annually for every 1,000 women in Sub-Saharan Africa (Figure 5.1). m) This birthspacing interval results from adding the nine months of pregnancy to the World Health Organization recommendation to wait at least 24 months before attempting pregnancy after giving birth. ${ }^{198}$

n) Chad, Democratic Republic of the Congo, Gabon and the Republic of Congo.

FIGURE

\section{1}

The rate of unintended pregnancy is highest, and the proportion of unintended pregnancies that end in abortion is lowest, in Middle Africa.

Rate of unintended pregnancies*

120

100

80

60

40

40

20

0

Sub-Saharan Africa
99

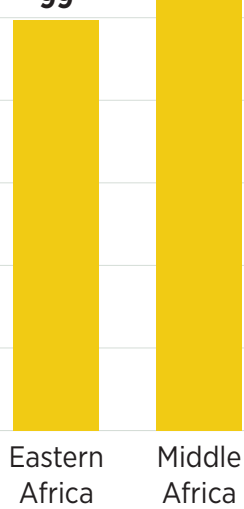

106

84

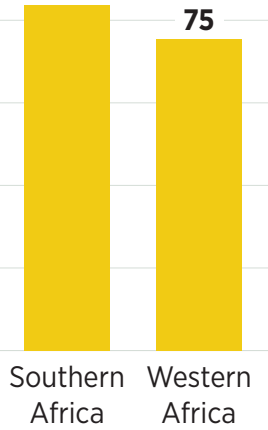

$\%$ of unintended pregnancies

100

80

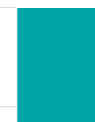

63

60

40

20

0 Sub-Saharan
Africa
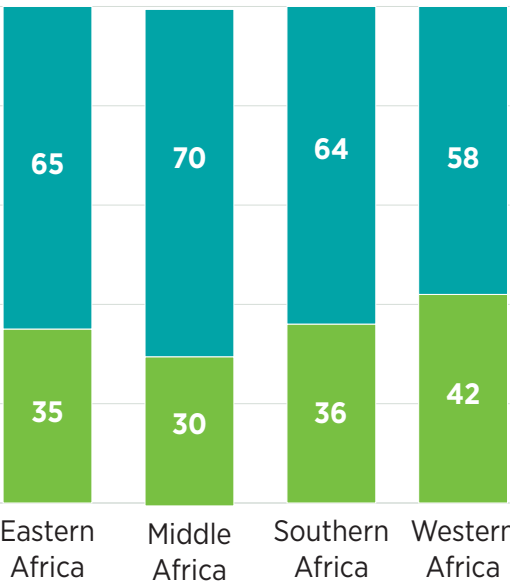

Southern Western Africa Africa

Ending in unplanned birth or miscarriage 
Women

interrupt a significantly higher

proportion of

unintended

pregnancies

now than

they did three

decades ago.

o) Traditional method use

among married women is nearly nonexistent $(0.5 \%)$ in Southern

Africa and is at 3-4\% in Eastern

and Western Africa. ${ }^{204}$
Unintended pregnancies include those that are mistimed (occurring before a woman wants to become pregnant) and those that are unwanted (occurring after she has completed her family). Expressed as a proportion, $42 \%$ of pregnancies occurring each year in Sub-Saharan Africa are unintended, the lowest of any world region.?

By subregion, unintended pregnancy rates divide into two main groupings: Eastern and Middle Africa share relatively high rates (99 and 106 per 1,000, respectively), and Western and Southern Africa have lower rates (75 and 84 per 1,000). The somewhat lower rates of unintended pregnancy in Western and Southern Africa mask large differences in the components that contribute to unintended pregnancy: Compared with women in Western Africa, those in Southern Africa have a far lower annual pregnancy rate (130 vs. 230 per 1,000) but are far more likely to experience an unintended pregnancy (65\% of pregnancies among women in Southern Africa are unintended vs. $33 \%$ of the pregnancies among women in Western Africa).

Once faced with an unintended pregnancy, what women do reflects their motivation-and their ability-to resolve that pregnancy through abortion, as opposed to going on to have an unplanned birth. Overall, $37 \%$ of unintended pregnancies end in abortion in the region as of 2015-2019 (Figure 5.1). ${ }^{7}$ The proportion of unintended pregnancies ending in abortion is lowest among women in Middle Africa (30\%), meaning that they likely have the most unplanned births. This proportion is somewhat higher among women in Eastern and Southern Africa (35\% and 36\%, respectively). Women in Western Africa resolve the highest proportion of unintended pregnancies through abortion (42\%), suggesting that exposure to the risks of unsafe abortion could be greater in that subregion than in any other, especially since no Western African country broadly allows abortion. ${ }^{20}$

In all four subregions-and indeed, the world over-women interrupt a significantly higher proportion of unintended pregnancies now than they did three decades ago. From 1990-1994 to 2015-2019, the proportion of unintended pregnancies resolved through abortion rose by $41 \%$ in Sub-Saharan Africa (see Supplementary Table online at https://www.guttmacher.org/report/fromunsafe-to-safe-abortion-in-subsaharan-africa). ${ }^{7}$ By subregion, these percentage increases were $26 \%$ in Middle Africa, 44\% in both Eastern and Western Africa, and $72 \%$ in Southern Africa.

\section{Use of modern contraceptives}

Modern contraceptive use is a proven way of preventing unintended pregnancies. All contraceptive methods have some rate of failure, but modern methods fail significantly less often than traditional methods. ${ }^{203}$ (See Data and Methods Appendix, page 39 , for definitions of modern and traditional contraceptive methods.) As of 2019, an average of $29 \%$ of married women in Sub-Saharan Africa use a modern method of contraception (Figure 5.2, page 31). ${ }^{204}$

This prevalence ranges from $14 \%$ and $20 \%$ in Middle and Western Africa to $57 \%$ in Southern Africa. Levels of traditional method use are mostly low, at $4 \%$ for the region, but Middle Africa has the highest rate of traditional method use, at $10 \% .^{\circ}$ As a result, married women in Middle Africa have a higher risk of unintended pregnancy from contraceptive failure than do married women in the three other subregions.

Relatively recent (2007 through 2018) country-level data on modern method use among sexually active unmarried women are available for 37 countries (Appendix Table 1, page 43). These women-who are at high risk for unintended pregnancy, and thus unsafe abortion - tend to use a modern method more than married women. ${ }^{205}$ The pattern holds for a very large majority of countries with data (32 of the 37). Modern contraceptive use among sexually active unmarried women is $2-3$ times that among married women in 16 of these countries, and it is five times that among married women in two countries. Yet the desire to avoid pregnancy among sexually active unmarried women is so strong that this level of modern method use is still not high enough to avoid high rates of unmet need, as discussed in the next section.

Married Sub-Saharan women are far better able to prevent unintended pregnancy now than they were in the recent past: The proportion who use a modern method more than tripled between 1990 and $2019,{ }^{204}$ from $8 \%$ to $29 \%$ (Figure 5.2, page 31 ). In the region's most populous subregion, Eastern Africa, the increase is particularly large: Modern method prevalence has risen fivefold since 1990. Increases have been more modest in Middle and Western Africa, the two subregions with the lowest current use. Southern Africa is an outlier, in both having a far higher starting point as of 1990 and plateauing at $54-58 \%$ as of 2000 .

\section{Unmet need for modern contraception}

Despite the increases in contraceptive use noted above, the rate at which women in Sub-Saharan Africa adopt modern methods is not keeping 
100

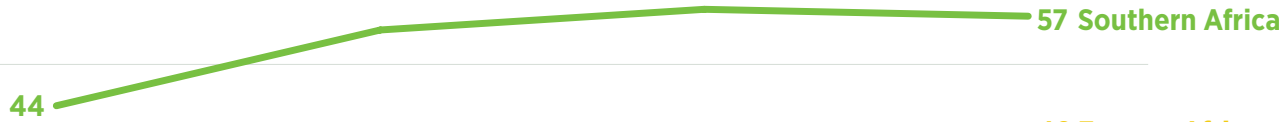

25

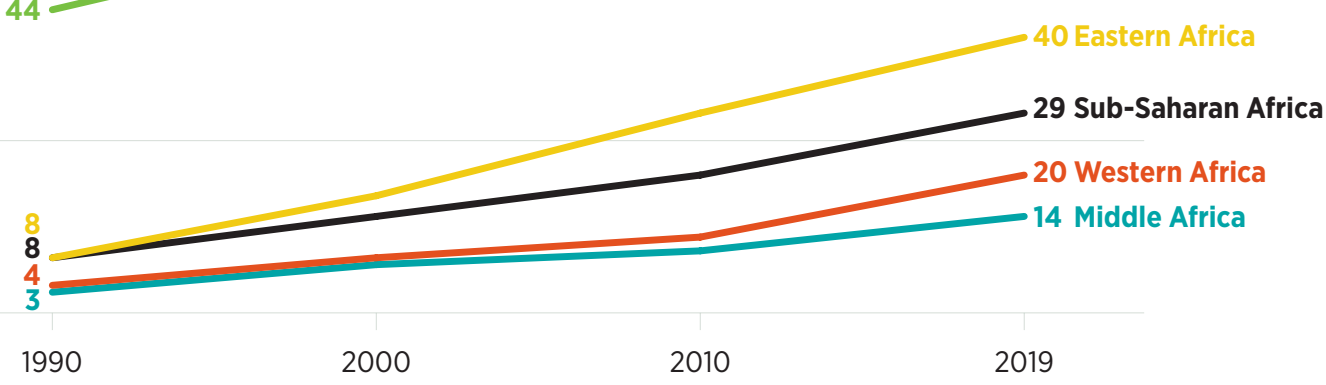

Note: Modern methods are female and male sterilization, oral contraceptives, IUDs, male and female condoms, injectables, implants, vaginal barrier methods, emergency contraception and the lactational amenhorrea method. Source: reference 204.

pace with rising demand. Unmet need for modern contraception refers to the proportion of women who want to avoid having a pregnancy but are not using a modern contraceptive method (i.e., those who are using a traditional method or are not using any method at all), and it measures the degree to which women are able to act on preferences to avoid pregnancy. ${ }^{p}$ Eighty-nine percent of unintended pregnancies in Sub-Saharan Africa occur among women with unmet need, and the remaining $11 \%$ occur among women whose modern method failed. ${ }^{84}$

Overall, slightly more than one-quarter (27\%) of married women in Sub-Saharan Africa have an unmet need for modern contraception, and because women in the region still desire relatively large families, the majority of unmet need is for methods to space births rather than to limit them. ${ }^{204}$ Southern Africa has the lowest proportion of women with unmet need in the region (15\%), because so many married women use a modern method and have since 2000. Eastern Africa stands out for having made the most progress: Unmet need for modern contraception declined by nearly one-third there since 2000. In contrast, there was virtually no change in unmet need among married women since 2000 in Middle Africa, the subregion where unmet need is highest.

Sexually active unmarried women in the region have higher levels of modern method use than do married women, but their desire to avoid pregnancy still outstrips their ability to act on that desire. For example, in one-third of the 36 countries with data, roughly one-half or more of sexually active unmarried women (48-64\%) do not use a modern method despite not wanting a pregnancy. ${ }^{91}$ What is more, unmet need for modern contraception is nearly always higher among sexually active unmarried women than among married women, a pattern found in all four subregions (see Figure 5.3, page 32 , for nonrepresentative examples from three countries in each subregion). This situation leaves sexually active unmarried women highly vulnerable to unintended pregnancy, and thus unsafe abortion, given the high costs of nonmarital childbearing in Sub-Saharan Africa.

Current levels of unmet need do not tell us whether women recently stopped using a modern method. In fact, many women discontinue use of a method without switching to a new one, which leaves them vulnerable to unintended pregnancy. p) It is assumed that all women categorized as having unmet need would want to use contraception, but this may not be so. In fact, some may be opposed to it, but there is no way to quantify this. The extent to which the risk of unintended pregnancy and its associated outcomes enter into their decision not to use a method is also unknown. 
$\%$ of women with unmet need for modern contraception

100

75

Sexually active unmarried*

Married

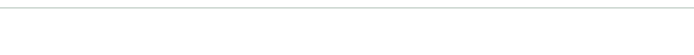

50

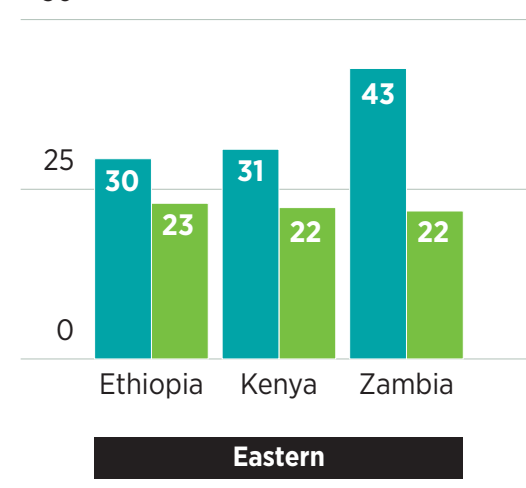

45 39

\section{8}

64

64

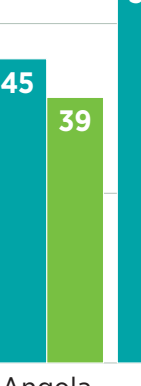

Angola

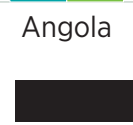

58

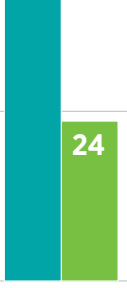

Chad
Middle

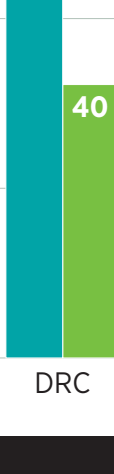

\section{Married}

*Those who had sex in the past four weeks. Notes: Selection of countries for inclusion is not meant to imply that they are representative of their subregion. Unmet need for modern contraception is the proportion of women who do not want to have a child for at least two years but are not practicing contraception or are using a traditional method. Modern methods are female and male sterilization, oral contraceptives, IUDs, male and female condoms, injectables, implants, vaginal barrier methods, emergency contraception and the

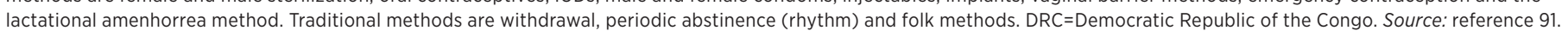

q) Botswana, Ethiopia, Kenya, Malawi, Rwanda, South Africa, Tanzania and Zambia.
Discontinuation rates for short-acting methods are relatively high in Sub-Saharan Africa. For a range of reasons, nearly half (47\%) of all modern method users in the region use the injectable. ${ }^{194}$ This region is unique in disproportionately relying on a single method; the method mix in other major world regions is made up of a much wider range of shortand long-acting modern methods.

The use of emergency contraception, which prevents pregnancy after unprotected intercourse, is often a one-time incident and thus is not accurately reflected in measures of general contraceptive use. The availability and use of this method should be more widespread in a region that has very high rates of sexual violence ${ }^{206}$ and in which $52 \%$ of women live where there is no legal exception for pregnancies resulting from rape. ${ }^{20,24}$ Emergency contraception is part of the United Nations Minimum Initial Service Package (MISP) for rape victims ${ }^{207}$ and should be the first therapeutic opportunity offered to victims if no more than five days have passed since the assault. ${ }^{31}$ (Although eight Sub-Saharan countries ${ }^{q}$ include provision of emergency contraception in their protocols for care following rape, ${ }^{206}$ no study has documented how often these protocols are followed.) The MISP for rape victims also covers humanitarian emergencies that uproot communities and disrupt norms of care. ${ }^{208}$ The Democratic Republic of the Congo ${ }^{209}$ and Somalia ${ }^{210}$ are especially affected, and women displaced by conflict remain highly vulnerable to unintended pregnancy and thus to unsafe abortion.

\section{Who experiences unintended pregnancies} Because of longstanding cultural expectations that childbearing should occur only within marriage, the vast majority of pregnancies among unmarried women in Sub-Saharan Africa are likely to be unintended. Data to back up this supposition are lacking, however, because nonmarital pregnancies are substantially underreported. ${ }^{179,211}$ Since most women in Sub-Saharan Africa are married by their early 20s (with the important exception of those in Southern Africa), ${ }^{212}$ young women who have yet to 
$\%$ of women aged 15-19 who are unmarried

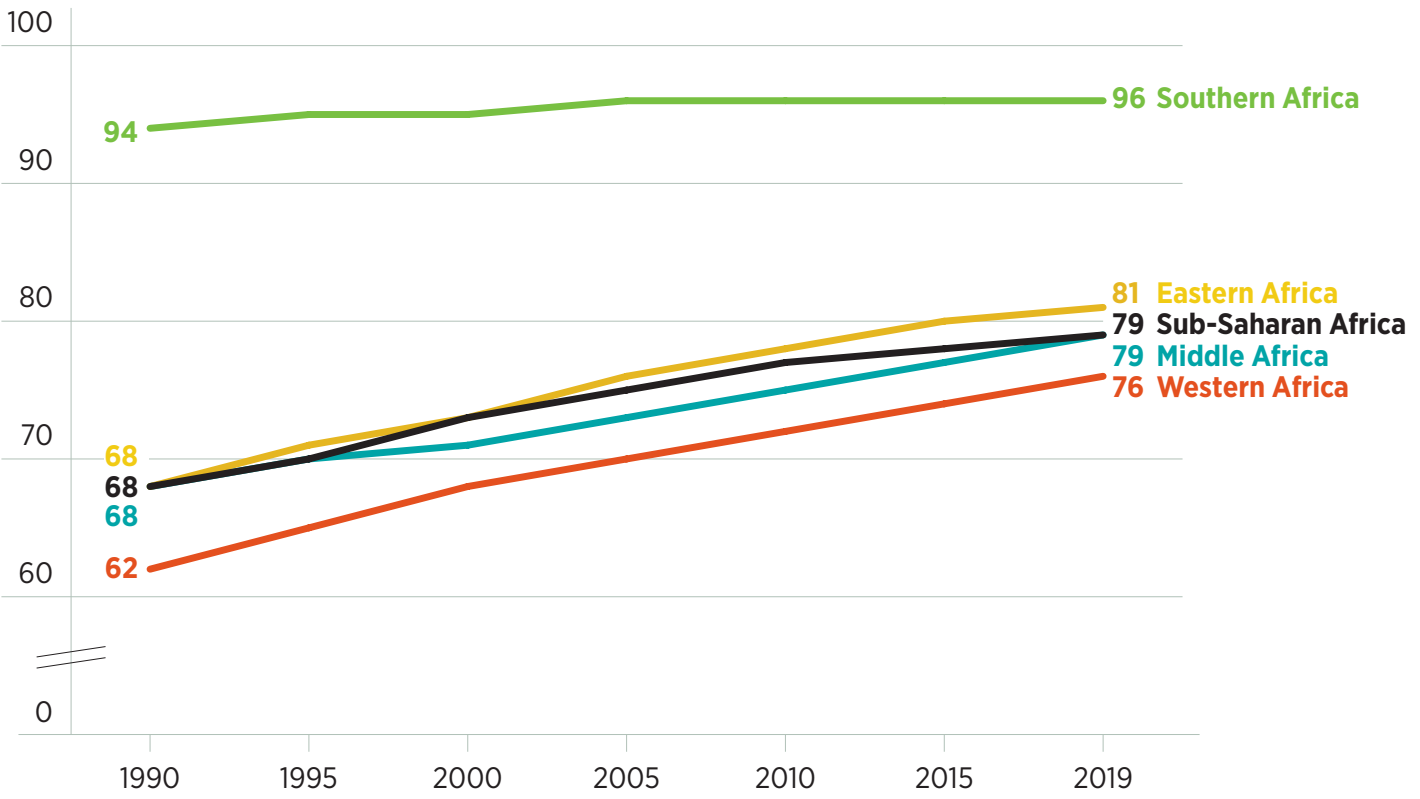

Source: reference 212 .

marry account for the bulk of nonmarital pregnancies. The certainty of stigma against nonmarital childbearing means that they often see abortion as their only option to avoid a harsh (and possibly violent) reaction from family and community. ${ }^{99,213,214}$

Positive economic and social changes are leading adolescent women in the region to increasingly postpone marriage - that is, when they have the agency to do so. Many women below the age of legal majority still have no say in this decision and are married off by their parents. Marriage before age 18 is fairly prevalent in Sub-Saharan Africa, especially in Middle and Western Africa: According to survey data from $2010-2016$, roughly $40 \%$ of 20-24-year-old women were married before age 18 in Middle and Western Africa, a combined subregion with both the highest prevalence of child marriage and slowest progress toward eradicating it. ${ }^{215}$

Delaying marriage often coincides with growing desires to stay in school and to be better prepared for labor force participation. For example, the proportion of young women enrolled in secondary school in the region nearly doubled between 1998 and 2017 (although this proportion is still relatively low, at about one-third). ${ }^{216}$ Evidence of this trend toward later marriage is clear in the increasing proportion of adolescents who are unmarried, ${ }^{r}$ which rose from $68 \%$ in 1990 to $79 \%$ in 2019 (Figure 5.4).

A rising age at first marriage can extend the period of risk for pregnancy outside of marriage, and therefore the risk of an unsafe abortion if young women happen to live where abortion is legally restricted. However, the length of this exposurethat is, the time between when a woman first has sex and when she first marries-is highly variable. ${ }^{217}$ First sex and first marriage virtually coincide in several countries (e.g., Chad, Comoros, Eritrea and Niger). On the other hand, the gap between first sex and first marriage-and thus the period of especially high risk of unintended pregnancy-has grown to between 3.5 and 5.0 years in five countries (Angola, Côte d'Ivoire, Gabon, Ghana and the Republic of Congo). r) The small numbers who are separated, widowed or divorced are included in this overall proportion of currently unmarried 15-19-year-olds. 


\section{(6) Conclusions and Recommendations}

Restricting

abortion by

law does not

eliminate it or

make it rare.

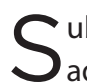
ub-Saharan Africa has made great strides in addressing unsafe abortion since 2000 . As of this writing, we simply do not know whether (or by how much) the novel coronavirus has undone any of this recent progress. Ideally, expansions of legal grounds in 21 of the region's countries will make abortion safer and more accessible, at least under the letter of the law. Reforming laws that regulate a highly stigmatized action takes time and may follow years of using multidisciplinary strategies to highlight the public health consequences and costs of unsafe abortion. The inclusion of abortion in the African Union's women's rights protocol, the Maputo Protocol, likely helped speed up the process. Sixty-one million Sub-Saharan African women now live in countries that allow abortion on at least the legal grounds prescribed in Article 14 of the Maputo Protocol. ${ }^{11,20-24}$ Enforcing the protocol, which is designed to save lives and protect the rights of African women, would have a profound impact. $^{4}$

It is important to underline that legality does not equal availability or access. Most of the 15 countries compliant with the Maputo Protocol (i.e., those in Category 4 or higher, which allow abortion in cases of rape, incest and grave fetal anomaly) still need to improve implementation and make legal and safe abortion services fully accessible for all women who need them. Although expanding grounds to comply with the protocol is an undeniable step forward, it serves at best as a foundation to build on. The "certain conditions" of abortion provided for in Article 14 do not necessarily reflect the actual grounds on which the vast majority of women seek abortions.

Evidence collected over decades demonstrates that women will seek abortions no matter the grounds specified by law. ${ }^{6}$ Most often, this means having to resort to clandestine abortions under conditions that may not meet clinical standards. According to the most recent regional estimates, which are for 2010-2014, Sub-Saharan Africa has the highest proportion by far of abortions estimated to be least safe (i.e., involving both a nonrecommended method and an untrained provider): Nearly half of abortions each year fall under the least-safe category. ${ }^{2}$ Middle Africa's level of least-safe abortions is the highest in Sub-Saharan Africa-and in the world-reaching 69\%.

The above is unsurprising, given the direct link between legality and safety. ${ }^{5}$ More than nine in 10 women of reproductive age in the region (92\%) live in countries where abortion is highly or moderately restricted. Even among the $8 \%$ of Sub-Saharan women who live where abortion is broadly legal, the strength of stigma and widespread lack of knowledge about legal criteria mean that many women still seek clandestine services. For most women living where abortion is legally restricted, those who are economically well off and live in urban areas are much more likely than less-privileged rural women to know about and be able to afford safe clandestine services. ${ }^{165}$ Poor women can usually only afford to go to unskilled providers or to self-induce using potentially unsafe methods. This situation defines the current context in which women increasingly resolve unintended pregnancies through abortion-26\% of unintended pregnancies in SubSaharan Africa ended in abortion as of 1990-1994, but $37 \%$ did so as of 2015-2019.' Unless the legal situation changes, or the safety of clandestine abortion improves, the health of increasing numbers of women will be at risk from unsafe abortions.

Recent evidence of how often Sub-Saharan women obtain abortions confirms that restricting abortion by law does not eliminate it or make it rare. As of 2015-2019, an estimated 33 abortions occur per 1,000 women of reproductive age annually, an overall rate that has remained virtually unchanged since 1995-1999. Even with unchanging rates, the actual numbers of abortions will still go up, simply because the number of Sub-Saharan women of 
reproductive age continues to grow. As a result, every year, more women will need postabortion care, the quality and reach of which is already poor in many countries.

Postabortion care is part of obstetric emergency care. Not being able to obtain it, or obtaining it too late, can turn morbidity into mortality: As of 2019, deaths related to unsafe abortion account for $7 \%$ of maternal mortality in Sub-Saharan Africa, ${ }^{84}$ and Sub-Saharan Africa's overall maternal mortality ratio is the highest of any world region, at 542 maternal deaths per 100,000 live births. ${ }^{218}$ SubSaharan governments that have committed to the United Nations' Sustainable Development Goal target of bringing maternal mortality down to 70 maternal deaths per 100,000 live births by $2030^{9}$ can make progress toward that goal by making abortion safer.

Nearly all of the harm caused by unsafe abortion is preventable. Eliminating unsafe abortion starts by expanding the availability of contraceptives, and thus their use, to prevent the unintended pregnancies that underlie the vast majority of abortions. While the rate of unintended pregnancy in Sub-Saharan Africa has fallen significantly over the past three decades, this rate remains by far the highest of any world region-mainly because overall pregnancy rates are highest in Sub-Saharan Africa. ${ }^{7}$ The most direct way to continue the decline in unintended pregnancy, which would lead to a concomitant decline in unsafe abortion, is to increase modern contraceptive use among women who say they do not want a pregnancy any time soon. Currently, a little more than one-quarter of married women say they want to avoid pregnancy but are not using a modern method.

How can Sub-Saharan governments improve the current situation and help women and couples prevent both unintended pregnancies and the unsafe abortions that often result? The following recommendations fall into three mutually reinforcing broad areas: laws and policies; services and counseling; and information, training and education. We also present some recommendations for future research (Box 6.1).

\section{Laws and policies}

- To harness the momentum of recent reform, the expansion of legal grounds that has started in several countries should be continued (or where it has stalled, it should be taken up again). The evidence-based connection between expanded legal grounds and improved safety should be widely disseminated, to garner strong support from professional medical bodies, ministries of health and broad-based community groups.

- Quality abortion services should be made available and accessible wherever they are allowed by law. It is also essential that governments enact accompanying policies and guidelines that fully implement the law with minimal administrative barriers. Individual countries now have many templates of implementation strategies from the region to choose from.

- Governments should lift the most egregious barriers to legal services, such as requirements that multiple physicians authorize abortions and that only physicians can provide them. A commonsense solution to the scarcity and expense of high-level physicians is to rewrite laws to permit the full range of health professionals to provide abortions. ${ }^{183}$ Providers who claim conscientious objection without offering the legally required referral to a willing provider can also affect availability; these abuses need to be prevented, so women always have access to a legal service.

- Sub-Saharan Africa is similar to other regions with restrictive abortion laws in that many countries spell out the grounds for when abortion is not punishable in national penal codes. But it is unique in belonging to a regional body, the African Union, which issued a women's rights protocol with legal grounds for abortion. Being fully compliant with the Maputo Protocol is a

\subsection{Research recommendations}

Studies on the following topics are urgently needed:

- Accurate measurement of the incidence of abortion, a deliberately hidden and underreported event

- Quality of abortion care and obstacles to getting legal abortions

- Postabortion care quality and accessibility

- Clandestine abortion practices and the social, health and economic consequences of unsafe abortion

- Misoprostol use, including where women obtain it, whether they receive accurate instructions on its use and its impact on morbidity and deaths

- Abortion stigma and its consequences, including standardization of measures of stigma, and design and testing of stigma-reducing interventions

- Inequities in the provision of contraceptive services and interventions to address them 
As long as legal restrictions and stigma exist, many abortions will continue to be unsafe. minimum international standard that all countries in the region should meet-and the nine countries that have still not ratified the protocol need to do so.

\section{Services and counseling}

\section{Safe abortion care}

- Sub-Saharan countries should closely follow WHO recommendations for expanding access by shifting tasks from scarce physicians to more plentiful midlevel professionals, given the region's dire human resources constraints. ${ }^{183}$

- Where national law permits abortion, legal services must be provided to the fullest extent of the law. Developing, disseminating and implementing national service guidelines that are consistent with the latest $\mathrm{WHO}$ recommendations would ensure that legal abortions have the benefit of evidence-based safety standards. ${ }^{3}$

- Providing safe abortions to the fullest extent of the law is always far less costly than treating women who present for care following an unsafe one. Safe services must include comprehensive counseling and postabortion contraception to help women avoid another unintended pregnancy.

- All health care professionals who provide abortion must be trained in WHO-recommended techniques, and the use of dilation and curettage must be discontinued without delay. ${ }^{183}$ This invasive and costly method should be completely replaced by either medication abortion or vacuum aspiration. ${ }^{3}$

- The use of medication abortion - the combination regimen of mifepristone and misoprostol when available, and misoprostol alone when it is not-is as safe as vacuum aspiration for firsttrimester abortion. ${ }^{3,149}$ Its use should be extended, since medication has the added benefit of often being preferred by providers (who appreciate it as less hands-on than surgical abortion) and by women (because of the privacy that it affords and its natural process).

- In countries where the law forces most abortions to be clandestine, governments and nongovernmental organizations should consider the harm-reduction strategies for misoprostol use that have worked in other parts of the world with restrictive abortion laws. For example, countries could follow examples from Latin America and set up strategies to share information about how to use misoprostol. ${ }^{219}$ Such strategies need to be adapted to take advantage of whatever technology-e.g., mobile phone platforms-would work best in the Sub-Saharan African context.

\section{Postabortion care}

- As long as legal restrictions and stigma exist, many abortions will continue to be unsafe, so postabortion care for the consequences of unsafe abortion will be needed. As with the provision of safe abortion, provision of postabortion care should follow updated WHO guidelines on how to manage incomplete abortions and other medical complications of unsafe abortions.

- Unlike the provision of abortion, the provision of postabortion care is always legal and, according to the International Federation of Gynecology and Obstetrics, medical practitioners are ethically bound to provide it. ${ }^{168}$ Laws that require providers to report abortions not meeting legal criteria clearly violate this professional obligation, as well as patients' right to confidentiality, and should be removed from the books.

- Evidence shows that nurses and midwives are capable of providing quality postabortion care, ${ }^{183}$ so training them in its provision and authorizing them to provide it would yield multiple benefits. These professionals are far more plentiful than physicians and are much more often based at primary care facilities, which are accessible to rural women.

- Incomplete abortion should be treated only with misoprostol or vacuum aspiration. Ministries of health need to ensure the consistent availability of these commodities. Any current use of D\&C for postabortion care, which is still extensive in several Sub-Saharan countries, should stop.

- The very high abortion rates among sexually active adolescents in the few countries with data suggest that these women shoulder a disproportionate burden of unsafe abortion. But the very stigma that prevents adolescents from accessing contraceptives to prevent unsafe abortion also prevents them from seeking out and receiving postabortion care. The need for youth-friendly, destigmatized postabortion services is thus crucial to address the fear of harsh judgment that is behind adolescents' reluctance to seek this essential lifesaving care. ${ }^{103,109,179}$

- Contraceptive services and counseling must be integrated into postabortion care, so that women can leave the site of care with an effective means to prevent unintended pregnancy in the future. 


\section{Contraceptive services}

- Improving women's ability to prevent the pregnancies they say they do not want will go a long way toward reducing the unsafe abortions that often follow. Unmet need for modern contraception is nearly always higher among sexually active unmarried women than among married women. Accessible and affordable contraceptive services should be nonjudgmental, so those who need them are not deterred from seeking them.

- Currently, the bulk of unmet need for modern contraception in Sub-Saharan Africa is among women wanting to space births. ${ }^{84}$ However, as preferences for smaller families take hold in the region, unmet need will increasingly be for methods to limit births. To meet current and future needs, national family planning programs must provide women with a range of methods that meet both types of needs.

- Women in Sub-Saharan Africa rely disproportionately on one contraceptive method, the injectable. It is important that women and couples have sufficient information about and access to the full range of effective methods, to maximize their options. Currently, the use of long-acting reversible contraceptive methods, which provide long-term effective protection, is very low in Sub-Saharan Africa. There is much room for growth in their provision, especially at the site of postabortion care..$^{220}$

- Improving timely access to emergency contraception is crucial, especially in the context of rape, since of the 38 countries in the four intermediate categories on the legality continuum, 14 do not specifically allow abortion for pregnancies resulting from rape. ${ }^{20}$

\section{Information, education and training}

- Law reform means little unless new criteria for legal abortion are widely communicated to the general public, medical professionals, legal professionals and law enforcement officials. Abortion is such a stigmatized subject that reluctance to talk about it publicly is at least part of the reason why women remain largely uninformed about legal criteria. Better public information campaigns are needed to more widely inform all parties of their rights and responsibilities.

- Abortion-related stigma compels many women to risk their health by seeking abortion outside of formal medical channels. This stigma touches both women who have abortions and those who seek postabortion care, as well as the medical professionals involved. Providing these professionals with values clarification and sensitivity training is an important start to their treating all patients without judgment and with dignity. Such training also helps medical professionals defend themselves from stigmatization for providing abortion-related services.

- Comprehensive sexuality education in school is essential for raising awareness among adolescents and young people of how to prevent pregnancy. Equally important is developing ways to reach out-of-school youth with this information. The very high rates of abortion among sexually active adolescents can fall only if young people are able to negotiate protected sex and obtain and correctly use contraceptives.

- In Sub-Saharan Africa, the severest abortion complications-as well as many abortion-related deaths-often stem from procedures performed after the first trimester. Thus, Ministries of Heath must provide training in safe methods of abortion in the second trimester and later, to widen the pool of personnel able to provide this service and save more lives.

\section{Moving from unsafe to safe abortion}

It is possible to accelerate progress toward ensuring that all women in Sub-Saharan Africa have access to the sexual and reproductive health services they need. Doing so will require scaling up the provision of contraceptive care, safe abortion care and postabortion care. Sustained collaboration from all stakeholders will be needed to expand legal criteria to at least the grounds in the African Union's Maputo Protocol. As the African Union's General Comment on Article 14 asserts, "the Maputo Protocol is the very first treaty to recognize abortion, under certain conditions, as women's human right which they should enjoy without restriction or fear of being prosecuted." ${ }^{10}$ Expanding legal criteria to align with this protocol demands input from all sectors of society.

Sub-Saharan Africa has made substantial progress toward talking openly about unsafe abortion, a subject that two decades ago was rarely broached in public discourse. Local and international actors have introduced safer and more effective methods of abortion; trained a wide range of providers in safer methods of abortion and postabortion care; enhanced the quality and coverage of postabortion care; and expanded the legal criteria for abortion to improve safety, thus reducing the numbers of women needing postabortion care. The incipient

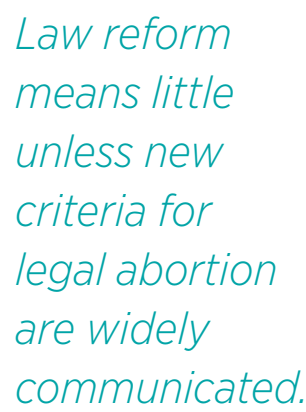


trend toward wanting smaller families and better control over healthy birthspacing is likely to continue. But without meaningful access to the means to have fewer children and more time between them, recourse to abortion is likely to increase in the short term.

Expanding the reach of modern contraceptive and postabortion services to all women who need them is crucial. Doing so cannot help but benefit Sub-Saharan Africa as a whole. The ability to have only wanted pregnancies enhances women's and infants' health, improves the status of women (for whom postponing starting a family often means more schooling and better job opportunities) and improves the overall financial well-being of families and national health systems. The consequences of inaction in preventing unsafe abortion are severe. More can and should be done to move the region forward toward fully protecting and enhancing women's reproductive health. 


\section{Data and Methods Appendix}

We use estimates derived by the Guttmacher Institute and World Health Organization in a comprehensive Bayesian hierarchical time-series model. ${ }^{7}$ The model's theoretical framework considers how the composition of women of reproductive age-and their contraceptive behaviors, fertility preferences and marital status-contribute to the incidence of unintended pregnancy and abortion. The model essentially applies the residual technique of estimating abortion in which the most "knowable" proximate determinants in the equation for fertility are rearranged to yield the "least knowable" proximate determinant, abortion. ${ }^{221}$ The model uses all available data on contraceptive need and use by marital status, and on unintended pregnancy and abortion among all women, to produce estimates for all 48 countries of Sub-Saharan Africa. It generates results at the regional and subregional levels for 1990-1994 through 2015-2019. ${ }^{\text {s7 }}$

The four principal proximate determinants (i.e., those that together define possible total fertility) are marriage (to the extent that it affects exposure to pregnancy), contraception, abortion and postpartum insusceptibility to conception. ${ }^{221}$ In the full global model, input data for 195 countries and territories cover 29 years (from 1990 through 2019). They include numbers of women of reproductive age and levels of contraceptive use and unmet need separately by marital status (as well as contraceptive method mix among married women). These input data come from a wide range of sources. The contraceptive, marital status and population numbers come from the United Nations Population Division. ${ }^{24,204,212}$

The total number of pregnancies is the sum of births, abortions and miscarriages. Data on the numbers of births are from the United Nations Population Division. Input data on abortion incidence come from official statistics (mainly for countries where abortion is legal under broad criteria) and-for countries with restrictive laws or incomplete official statistics-from country-specific studies and nationally representative surveys of women with estimates of abortion incidence. The numbers of miscarriages are derived from clinical studies that used life-table analyses in which miscarriages equal approximately $20 \%$ of births plus $10 \%$ of abortions. ${ }^{222}$

We break pregnancies down into those that are intended and unintended, and births into those that are planned and unplanned. Estimates of the proportions of births that are unplanned are from women's reports of the planning status of recent births in surveys conducted in the Demographic and Health Survey (DHS) program or in similar surveys. In these surveys, a birth is considered unplanned if it occurred two or more years sooner than desired (i.e., was mistimed) or if it was not wanted at all at the time of conception (i.e., was unwanted).

The current Bayesian model permits the exchange of information across time periods and geographic areas, statistical inference and the calculation of uncertainty intervals around all estimated values. It also enables estimations of trends by using the same evidence base and methodology for all time periods.

The model has some limitations. The variability in the quantity and quality of the underlying data are reflected in the uncertainty intervals provided with each result. More on the limitations of this approach has been published elsewhere. ${ }^{7}$ Stakeholders in the countries of the region likely continue to prefer country-specific studies to model-based estimates. However, accurate recordkeeping of abortion is still very rare, even in the four Sub-Saharan countries that have decriminalized abortion. Further, despite recent innovative research approaches that have improved the accuracy of country-level estimates of abortion, Bayesian modeling still likely produces the most s) An earlier Bayesian hierarchical model provided abortion incidence from 1990-1994 through 2010-2014. The estimates for 1990-1994 through 2015-2019 that we report here completely replace the differently modeled estimates that were published in 2016 and 2018. 
accurate regional and subregional abortion estimates, given the unavoidable uncertainty of these data.

\section{Country-specific abortion data and estimates}

Estimates of abortion incidence are presented in this report for a number of countries. These estimates are based on an indirect estimation approach, the abortion incidence complications method. ${ }^{223}$ The method uses the only available empirical data on abortion in many countries that legally restrict it (i.e., the numbers of women who obtain care for complications at medical facilities, collected in a nationally representative Health Facilities Survey). The methodology builds out from these measurable data to estimate the total number of abortions in a given year by interviewing experts familiar with the conditions of abortion in the country, in the Health Professionals Survey. These experts are asked a series of questions to allow estimation of the proportion of all abortions represented by those that caused complications and that are counted in the Health Facilities Survey. This indirect approach provides an approximate estimate of abortion incidence and has been described and assessed elsewhere. ${ }^{223}$

We obtained data on the characteristics of women who had an abortion, such as their age, marital status and how and why they had had an abortion, from nationally representative and small-scale surveys. Because of the scarcity of information describing abortion in these mostly restrictive countries, most of the limited data that we have on abortion methods and providers had to come from nonrepresentative surveys of women who developed complications and presented for postabortion care. Such surveys, especially the nationally representative Prospective Morbidity Surveys, provide information on the types and severity of complications that women experience.

These sources are not free of bias. Abortions are severely underreported in general, and surveys of women receiving postabortion care in particular suffer from misreporting, as many women understandably report complicated abortions as complicated miscarriages (the symptoms of which can be indistinguishable). Further, information from women on which abortion methods were used is often unreliable. For example, they may be given a tablet without knowing what it is or how to use it, a problem that is particularly challenging to solve when we understand that the use of misoprostol is increasing throughout the region, primarily in cities. $^{159-161}$

Additionally, when estimates are produced by region or area of residence, the approach may overstate the incidence of abortion for the places that women travel to-most often, metropolitan areas. Women often travel from their homes to cities for both abortion and postabortion services that are more accessible, of better quality and more likely to offer anonymity. Since it is not easy-or even possible-to determine where these women travel from, they are by necessity assumed to be residents of those metropolitan areas, which likely overstates the true incidence of abortion in metropolitan areas (usually capital cities). ${ }^{224}$

\section{Safety of abortions}

WHO's classification of safety uses three gradationssafe, less safe and least safe. ${ }^{5}$ The total unsafe is the sum of less- and least-safe abortions. An abortion is classified as safe if it meets two general criteria: It was induced 1) with a WHO-recommended method at the recommended gestation and 2) by an appropriately trained individual. Less-safe abortions are those that meet only one of these criteria, and leastsafe abortions are those that meet neither.

Another Bayesian hierarchical model distributes the annual number of abortions by safety for the period 2010-2014. ${ }^{5}$ Its predictors of safety are the availability of safe abortion methods, trained providers and facilities equipped to provide safe abortion; the strength of abortion stigma (which can influence women's levels of empowerment, autonomy and agency) and providers' willingness to perform abortions; and the overall quality of health services and health infrastructure.

\section{Safety model covariates}

The theoretical predictors for a given country were represented in the model by: the number of years that mifepristone has been registered; the proportion of the population that lives in urban areas; and the country's score on the gender inequality index, which is a composite measure comprising indicators of reproductive health, women's empowerment and economic status. ${ }^{225}$ The registration status of misoprostol for any indication in the country was also added as a covariate.

\section{Data inputs}

Researchers undertook a systematic search for the above data on methods used, the types of providers and the settings in which the abortion took 
place. Data came from national statistics, DHS and similar surveys, and other national and subnational studies. In all, data were analyzed for 182 countries and territories. The years 2010-2014 were first considered as cut-offs; however, information from 2008-2009 was used if more recent data were unavailable. National-level data were considered optimal, but subnational data were used if they were sufficiently reliable.

\section{National-level abortion laws}

The current legal status in the region's 48 countries is based largely on information assembled by the Center for Reproductive Rights (CRR); ${ }^{20}$ we also supplemented CRR's database with the actual penal codes and national-level decrees for three countries. ${ }^{t}$ Information on change over time comes primarily from overviews of legal reform from 1994 through $2014^{226}$ and from 2008 through 2019. ${ }^{227}$ The numbers and proportions of women of reproductive age to distribute across the six categories of legality come from country-specific population data for 2019 from the United Nations Population Division. ${ }^{24}$ We use six legality categories, even though CRR uses five, because CRR has just a single health category, which reflects WHO's holistic definition of health. However, we needed separate categories for physical health and for mental health to measure progress toward complying with the safe abortion grounds in the African Union's Maputo Protocol, which explicitly mentions mental health. ${ }^{\mathrm{u}}$ In addition, having a separate explicit mental health category allowed us to document movement from health only to physical and mental health (which occurred in three countries).

\section{Abortion mortality}

We calculated case-fatality rates for abortion in 2000 and 2019 using the Adding It Up approach. ${ }^{228}$ For 2019, we first obtained the number of country-specific maternal deaths for the year 2017. This involved computing the maternal mortality ratio-the number of maternal deaths per 100,000 live births-for 2017, using the number of maternal deaths from the database of the 2017 collaborative maternal mortality estimates made by five international agencies ${ }^{218}$ and the number of births from the United Nations Population Division. ${ }^{24}$ This ratio was then applied to the number of births in 2019 to estimate the number of maternal deaths per 100,000 births in 2019.

Second, we estimated the number of country-level deaths caused by complications of abortion. We began with cause-of-death estimates for 2017 for the region's 48 countries from the Institute for Health Metrics and Evaluation (IHME). Late maternal deaths (at least 42 days after the pregnancy ended) were excluded, to be consistent with WHO's definition of maternal mortality. IHME's estimates combine deaths from abortions and miscarriages; ${ }_{i}^{229}$ to calculate deaths from abortions only, we removed miscarriage-related deaths on the basis of the rate of one maternal death per 100,000 miscarriages at $14-27$ weeks' gestation. ${ }^{230}$ Applying this final proportion of deaths from abortion only to the number of maternal deaths in 2019 (based on Maternal Mortality Estimation Inter-agency Group estimates) resulted in the number of deaths from abortion for each of the region's countries.

Finally, we summed country-specific numbers to yield regional and subregional totals, which served as the numerators for abortion case-fatality rates. The denominator is the number of abortions in 2019 from the annualized average for 2015-2019 from the Bayesian hierarchical model, multiplied by $100,000 .^{72}$ The estimates of abortion case-fatality rates for the baseline year of 2000 were obtained following a similar process.

\section{Morbidity due to unsafe abortion}

Rates of treatment for abortion

\section{complications}

A study used nationally representative data from 26 developing countries, including 10 from SubSaharan Africa, to estimate regional and subregional rates of treatment for complications from unsafe abortion for 2012. ${ }^{169}$ The national estimates used data from published country-specific studies on postabortion care and country-level health systems data.

\section{Estimates of complicated abortions that} need medical care

We used information on the likelihood of needing and getting care for abortion complications from the Health Professionals Survey, one of the two surveys used in the abortion incidence complications method mentioned earlier. The health and other types of professionals who participated in the survey were selected on the basis of their expertise and knowledge of abortion in their country. They included medical providers (nurses, midwives and physicians) and other experts with informed perspectives (policymakers, researchers, advocates and specialists in public health services and programs). We show the results of Health Professionals Surveys from 10 countries; $^{\mathrm{v}}$ these surveys were carried out between 2008 and 2016. t) The three countries that we categorize differently than CRR does are Ethiopia, Mozambique and Rwanda. Thus, our placement of countries, and the related calculations of both countries and numbers of women of reproductive age within categories, differ slightly from those of CRR.

u) Article 14(2)c obliges member states to legalize abortion when the pregnant woman's life is at risk; to protect her physical or mental health; and in cases of rape, incest and grave fetal anomaly.

v) Burkina Faso, Ethiopia, Kenya, Malawi, Nigeria, Rwanda, Senegal, Tanzania, Uganda and Zimbabwe. 
Health Professionals Survey respondents were interviewed in person about their perceptions of abortion provision - the types of providers and methods that women use, women's risk of experiencing incomplete abortion and medical complications with each type of provider, the likelihood that women would obtain treatment at a facility if complications occur, and the costs of obtaining abortions and treatment.

Because abortion practices and outcomes vary widely by poverty status and place of residence, Health Professionals Survey respondents supplied information separately for each of four key subgroups. These subgroups are: poor urban women, poor rural women, nonpoor urban women and nonpoor rural women. Being poor was defined as living in a household with an income below the national average (e.g., in terms of minimum salary, in countries where this concept is commonly recognized); as having difficulties paying for basic necessities; or as having low educational attainment. Responses were averaged to provide an approximate profile for each of the four population subgroups, and averages were weighted by the population size of each subgroup to yield averages for the country as a whole. This national total was also used to create the multiplier to inflate the treated cases to the total number of abortions occurring in the country.

\section{Sexual and reproductive health indicators} Nationally representative DHS surveys on women's sexual and reproductive health were key sources of data. Total fertility rates, levels of contraceptive use and unmet need, and data on the planning status of births and the recency of sexual activity were obtained from the most recent survey for each country for which the majority of these indicators were available. The earliest survey dates from 2007, and we tried to update national surveys as new data became available. For time trends in modern contraceptive use, we relied on multiyear data from the United Nations' model-based estimates, which themselves draw on data from DHS and DHS-type surveys.

For this report, we use the DHS definitions of modern and traditional methods. Thus, we classify female and male sterilization, oral contraceptives, IUDs, injectables, implants, female and male condoms, emergency contraception, diaphragms, contraceptive foams and jellies, the lactational amenorrhea method, the standard days method, cervical caps and sponges as modern methods of contraception. Periodic abstinence (rhythm and calendar methods), withdrawal and folk methods are classified as traditional methods. Women with an unmet need for modern contraceptives are those aged 15-49 who are able to become pregnant but who want to avoid pregnancy and are not using a modern method. Unintended pregnancies are pregnancies that were unwanted or mistimed at the time of conception.

We needed to use two definitions of current sexual activity. For estimates of unmet need for modern contraception among sexually active unmarried women, we define being sexually active as having had sexual intercourse in the four weeks prior to the survey. However, to enable calculation of abortion rates among sexually active adolescents, we expanded this time frame to the 12 months preceding the survey, to ensure a large enough sample size for analysis. 


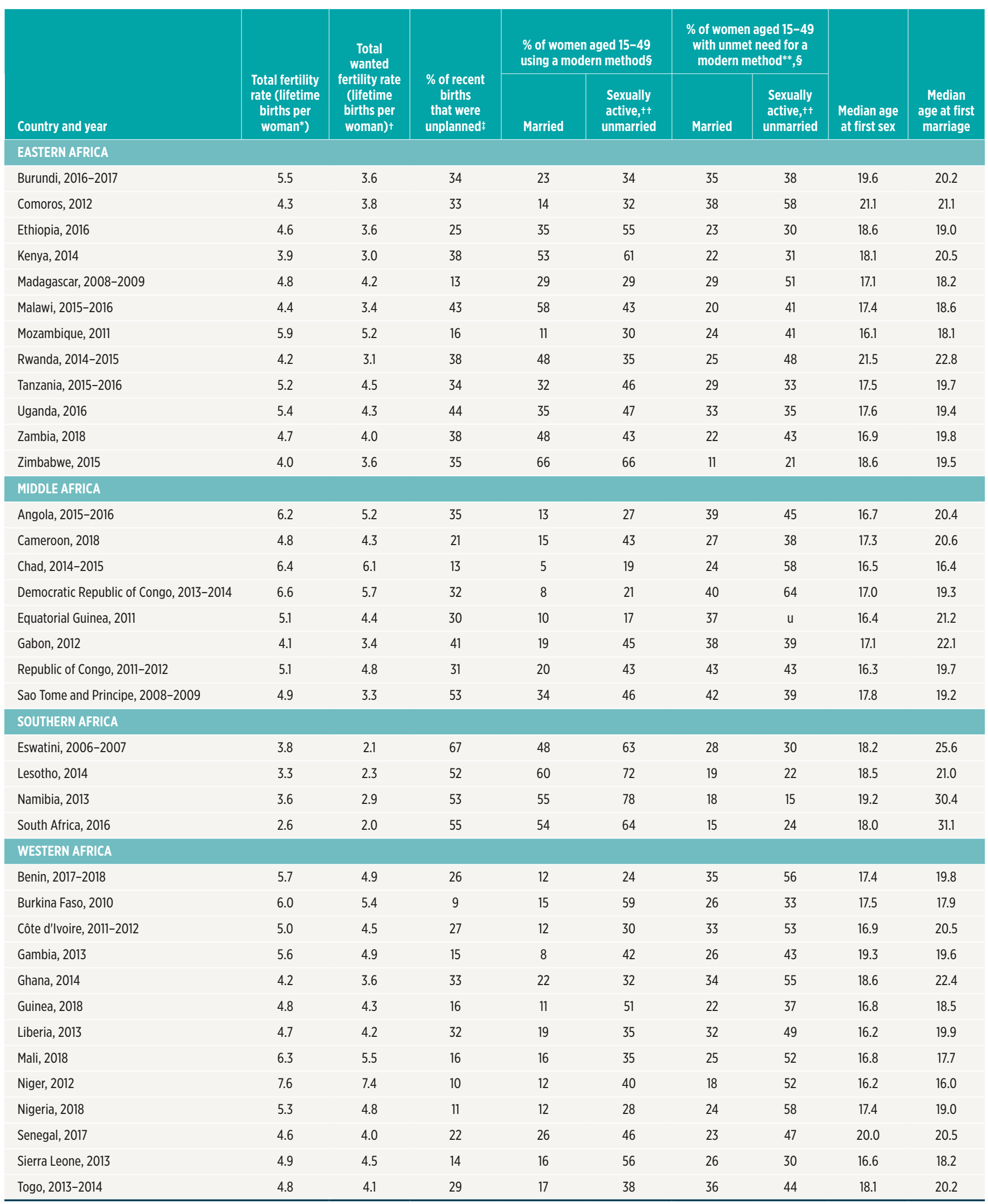

See next page for notes. 


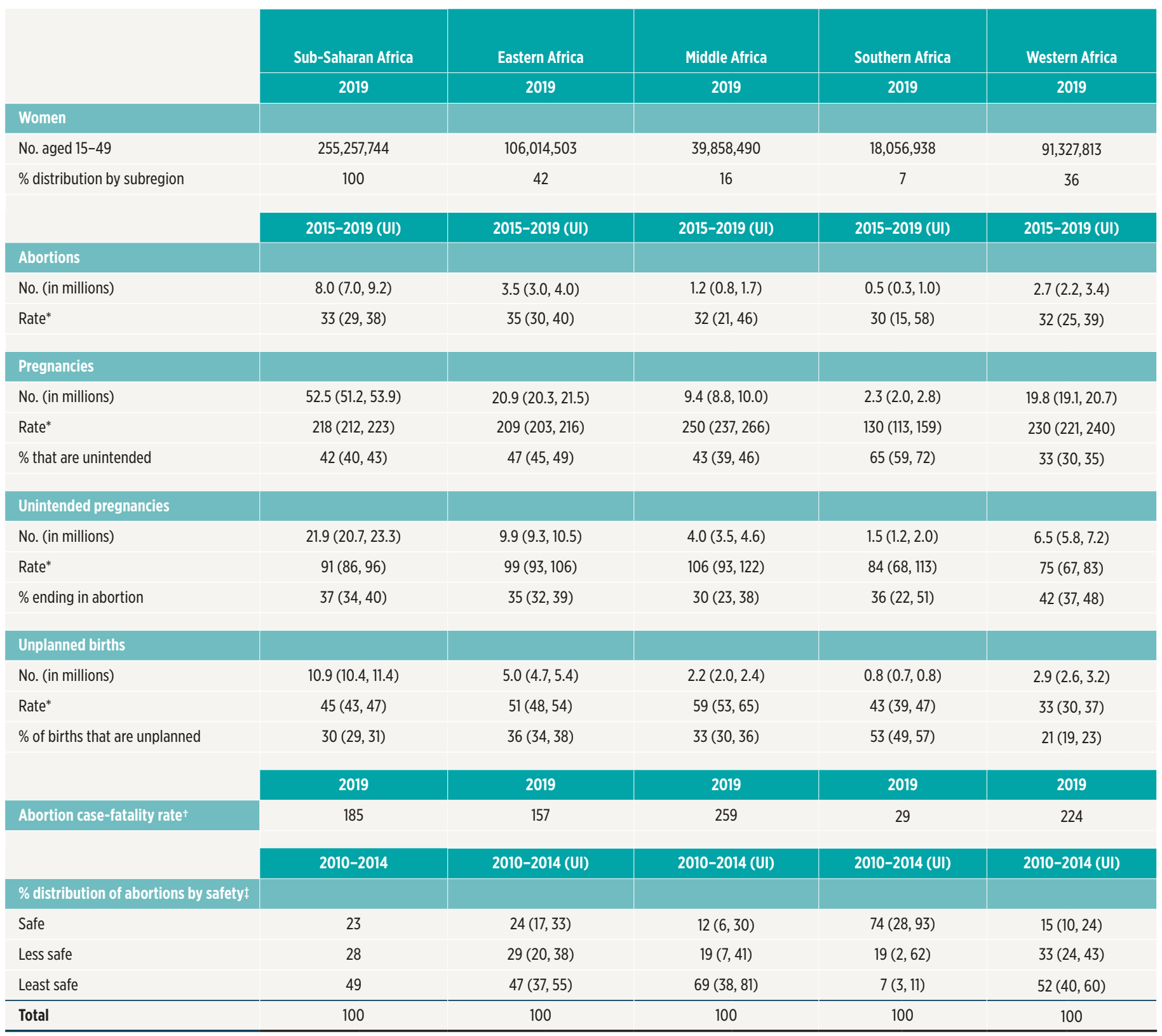

*Number per 1,000 women aged 15-49. †Number of maternal deaths per 100,000 abortions. $¥$ Safe procedures are those that are done by a trained provider and that are done using a World Health Organization-recommended method at the appropriate timing of pregnancy; less-safe abortions meet just one of these criteria; and least-safe abortions meet neither criterion. Note: Ul=uncertainty interval (unavailable for safety distribution for Sub-Saharan Africa as a whole). Sources: references 2, 5, 7, 8 and 24.

See supplementary table online for additional data years: https://www.guttmacher.org/report/from-unsafe-to-safe-abortion-in-subsaharan-africa

\section{Notes to Appendix Table 1, page 43}

*The number of births a woman would have, assuming that current age-specific fertility rates over the past three years remain the same over her lifetime. ${ }^{+}$The number of births a woman would have if she were to avoid births that exceed her stated ideal number. fFor births over the past three years, the sum of mistimed births (those that were wanted later) and unwanted births (those that were not wanted at all). §Modern methods of contraception include female and male sterilization, the pill, the IUD, the injectable, the implant, emergency contraception, male and female condoms, the lactational amenorrhea method, the diaphragm, spermicides and the standard days method. ${ }^{* *}$ The proportion of women who are able to become pregnant and who do not want a pregnancy within the next two years, but are not using a modern contraceptive method. +tHad sexual intercourse within the past four weeks. Notes: To ensure meaningful data and data comparability, median ages are calculated using the first age-group at which $50 \%$ of women had experienced both events, first sex and first marriage. That means we needed to use four base age-groups to calculate the medians for the this table. Those age-groups and countries are the following: 20-24 (Benin, Burkina Faso, Chad, Democratic Republic of Congo, Ethiopia, Guinea, Liberia, Madagascar, Malawi, Mali, Mozambique, Niger, Nigeria, Republic of Congo, Sao Tome and Principe, Sierra Leone, Tanzania, Uganda and Zimbabwe); 25-29 (Angola, Burundi, Cameroon, Comoros, Côte d'Ivoire, Equatorial Guinea, Gabon, Gambia, Ghana, Kenya, Lesotho, Rwanda, Senegal, Togo and Zambia); 30-34 (Eswatini); and 35-39 (Namibia and South Africa). u=unavailable. Sources: Special tabulations of DHS data sets, except for Cameroon (2018) and Equatorial Guinea (2011). Because we used the published reports rather than the data sets for these two countries, their planning status refers to births in the past five years and includes current pregnancies. 


\section{References}

1. Beegle $K$ et al., Poverty in a Rising Africa, Washington, DC: World Bank, 2016, doi:10.1596/978-1-4648-0723-7.

2. Special tabulations of data from Ganatra B et al., Global, regional, and subregional classification of abortions by safety, 2010-14: estimates from a Bayesian hierarchical model, Lancet, 2017, 390(10110):2372-2381, doi:10.1016/ S0140-6736(17)31794-4

3. World Health Organization (WHO), Safe Abortion: Technical and Policy Guidance for Health Systems, second ed., 2012, https://apps.who.int/iris/bitstream/ handle/10665/70914/9789241548434_eng.pdf;j.

4. Ngwena CG, State obligations to implement African abortion laws: employing human rights in a changing legal landscape, International Journal of Gynecology \& Obstetrics, 2012, 119(2):198-202, doi:10.1016/j.ijgo.2012.08.001.

5. Ganatra B et al., Global, regional, and subregional classification of abortions by safety, 2010-14: estimates from a Bayesian hierarchical model, Lancet, 2017, 390(10110):2372-2381, doi:10.1016/ S0140-6736(17)31794-4.

6. Singh S et al., Abortion Worldwide 2017: Uneven Progress and Unequal Access, New York: Guttmacher Institute, 2018, https://www.guttmacher.org/report/ abortion-worldwide-2017.

7. Bearak J et al., Unintended pregnancy and abortion by income, region, and the legal status of abortion: estimates from a comprehensive model for 1990-2019, Lancet Global Health, 2020, 8(9):E1152-E1161, doi:10.1016/ S2214-109X(20)30315-6.

8. Special tabulations of case-fatality rates based on data on deaths from abortions from the Global Health Data Exchange; number of maternal deaths from $\mathrm{WHO}$; and numbers of abortions from Bayesian model from http:// ghdx.healthdata.org/gbd-results-tool.

9. United Nations (UN), Sustainable Development Goals: Knowledge Platform, no date, https:// sustainabledevelopment.un.org/sdgs.

10. African Commission on Human and Peoples' Rights, General Comment No. 2 on Article 14.1 (a), (b), (c) and (f) and Article 14. 2 (a) and (c) of the Protocol to the African Charter on Human and Peoples' Rights on the Rights of Women in Africa, Luanda, Angola: African Union, 2014.

11. African Union, Protocol to the African Charter on Human and People's Rights on the Rights of Women in Africa, Maputo Protocol, July 11, 2003, https://www. un.org/en/africa/osaa/pdf/au/protocol_rights_women_ africa_2003.pdf.

12. African Union Commission, Plan of Action on Sexual and Reproductive Health and Rights (Maputo Plan of Action), Maputo, Mozambique: African Union, 2006.

13. UN Statistics Division, Methodology: standard country or area codes for statistical use, countries or areas/ geographical regions, 2019, https://unstats.un.org/unsd/ methodology $/ \mathrm{m} 49 /$.

14. Ngwena CG, Protocol to the African Charter on the Rights of Women: implications for access to abortion at the regional level, International Journal of Gynecology \& Obstetrics, 2010, 110(2):163-166, doi:10.1016/j. ijgo.2010.04.013.

15. Republic of Zambia, Termination of Pregnancy Act, Chapter 304 of the Laws of Zambia, 1972, No. Act 26 of 1972, amended by Act No. 3 of 1994.

16. República de Cabo Verde, Lei de Interrupcão Voluntária de Gravidez, Lei no. 9/III/86, Feb. 5, 1987.

17. República da Guiné-Bissau, Conselho de Estado, Decreto lei no 4/93, Código Penal, Boletim Oficial, 1993, (41), https://abortion-policies.srhr.org/documents/countries/ 02-Guinea-Bissau-Penal-Code-1993.pdf.

18. Assembleia Nacional, São Tomé e Príncipe Código Penal, Lei 6/2012, July 5, 2012, https://abortion-policies. srhr.org/documents/countries/01-Sao-Tome-and-PrincipePenal-Code-2012.pdf.

19. Parliament of the Republic of South Africa, Choice on Termination of Pregnancy Act, Law No. 92, 1996.

20. Center for Reproductive Rights (CRR), The world's abortion laws, no date, http://reproductiverights.org/ worldabortionlaws.

21. Assembleia da República de Moçambique, Lei da Revisão do Código Penal, Lei n. ${ }^{\circ}$ 35/2014, Dec. 31, 2014, https://abortion-policies.srhr.org/documents/countries/ 01-Mozambique-Penal-Code-2013.pdf.

22. Federal Democratic Republic of Ethiopia, Criminal Code, Law No. 414/2004.

23. Ministry of Health of Rwanda, Ministerial Order $\mathrm{N}^{\circ} 002$ Determining Conditions to Be Satisfied for a Medical Doctor to Perform an Abortion in Rwanda, Apr. 8, 2019, https://abortion-policies.srhr.org/documents/countries/10Rwanda-Order-on-abortion-MoH-2019.pdf.

24. Department of Economic and Social Affairs, UN Population Division, World Population Prospects, the 2019 Revision, 2020, https://esa.un.org/unpd/wpp/.

25. Statute Law of England and Ireland, Offenses Against the Person Act, 24 and 25 Victoriae, Cap. 100, Aug. 6, 1861.

26. Parliament of Zimbabwe, Termination of Pregnancy Act Act 22 (Orig. Act 29/1977), 2001, https://www.refworld.org/ docid/4c46c4de2.html.

27. Parliament de Cameroun, Loi Portant le Code Pénal, 2017/007, July 12, 2016, https://www.droit-afrique.com/ uploads/Cameroun-Code-2016-penal1.pdf.

28. Assemblée Nationale, Burkina Faso, Loi Portant le Code Pénal, May 31, 2018, https://lavoixdujuristebf.files. wordpress.com/2018/11/code-pc3a9nal-nouveau.pdf.

29. García-Moreno $C$ et al., Global and Regional Estimates of Violence Against Women: Prevalence and Health Effects of Intimate Partner Violence and Non-Partner Sexual Violence, Geneva:WHO, 2013, https://www.who.int/ reproductivehealth/publications/violence/9789241564625/ en/.

30. Palmer JJ and Storeng KT, Building the nation's body: the contested role of abortion and family planning in post-war South Sudan, Social Science \& Medicine, 2016, 
168:84-92, doi:10.1016/j.socscimed.2016.09.011.

31. WHO, Guidelines for Medico-Legal Care for Victims of Sexual Violence, 2003, https://apps.who.int/iris/ bitstream/handle/10665/42788/924154628X.pdf;jsession$\mathrm{id}=2 \mathrm{~B} 6 \mathrm{~A} 91114211 \mathrm{BA} 5238 \mathrm{C} 107 \mathrm{~B} 4 \mathrm{~F} 4 \mathrm{ACBD} 2 \mathrm{D}$ ? sequence $=1$.

32. Scott $J$ et al., Respondent-driven sampling to assess mental health outcomes, stigma and acceptance among women raising children born from sexual violencerelated pregnancies in eastern Democratic Republic of Congo, BMJ Open, 2015, 5(e007057), doi:10.1136/ bmjopen-2014-007057.

33. Hodes $R$, The culture of illegal abortion in South Africa, Journal of Southern African Studies, 2016, 42(1):79-93, doi:10.1080/03057070.2016.1133086.

34. Mahadew RA, Mauritius decriminalizes abortion in certain circumstances-lessons from the Travaux preparatoires, Statute Law Review, 2015, 36(2):160-174, doi:10.1093/slr/hmv005.

35. Martin M, Welcome address to CEDAW, Mauritius, presented at the Quatre Bornes, Mauritius, Sept. 11, 2012.

36. Maiga $M$, Bref aperçu sur la loi sur la santé de la reproduction en Afrique de l'Ouest et du Centre, paper presented at the Rencontre Régionale, Afrique Francophone, Advance Family Planning, Burkina Faso, Mar. 14, 2014, https://www.advancefamilyplanning.org/ sites/default/files/2017-09/LA\%20LOI\%20SR\%20avec\%20 Exemple.pdf.

37. République du Sénégal, Loi Relative à la Santé de la Reproduction, Loi n² 2005-18, Aug. 5, 2005, https://www. prb.org/wp-content/uploads/2018/05/Loi-n\%C2\%BO2005-18-du-5-Aou\%CC\%82t-2005-Relative-a\%CC\%80-laSante\%CC\%81-de-la-Reproduction.-Se\%CC\%81ne\%CC\%81gal.pdf.

38. République de Madagascar, Loi Fixant les Règles Générales Régissant la Santé de la Reproduction et la Planification Familiale, Loi n ${ }^{\circ}$ 2017-043, Dec. 13, 2017, http://genderlinks.org.za/wp-content/uploads/2019/04/ Sexual-Education_Madagascar_Adolscent-SRHR_2017.pdf.

39. Republic of The Gambia, Office of the Vice President and Ministry for Women's Affairs, Women's Act, 2010.

40. Republic of Kenya, Constitution of Kenya, 2010.

41. Federal Republic of Somalia, Provisional Constitution, Aug. 1, 2012.

42. African Union, List of countries which have signed, ratified/acceded to the protocol to the African Charter on Human and People's Rights on the Rights of Women in Africa, 2019, https://au.int/sites/default/ files/treaties/37077-sI-PROTOCOL\%20TO\%20THE\%20 AFRICAN\%20CHARTER\%20ON\%20HUMAN\%20AND\%20 PEOPLE\%27S\%2ORIGHTS\%20ON\%20THE\%20RIGHTS\%20 OF\%20WOMEN\%20IN\%20AFRICA.pdf.

43. Cabinet du President de la République Démocratique du Congo, Protocole à la charte Africaine des droits de I'homme et des peuples, relatif aux droits de la femme en Afrique, Journal Officiel de la République Démocratique du Congo, 59 année, Numéro spécial.

44. Cabinet du Président de la République Démocratique du Congo, Circulaire relative à la Mise en exécution des dispositions de l'Article 14 du Protocole à la charte africaine des droits de l'homme et des peuples relative aux droits de la femme en Afrique, Journal Officiel de la République
Démocratique du Congo, 59 année, Número spécial, June 5 , 2018.

45. Republic of Rwanda, Organic law instituting the penal code, Official Gazette, 2012, Organic Law N ${ }^{\circ} 01 / 2012 / O L$ of 02/05/2012(Special Issue).

46. Hodoglugil $\mathrm{N}$ et al., Making abortion safer in Rwanda: operationalization of the penal code of 2012 to expand legal exemptions and challenges, African Journal of Reproductive Health, 2017, 21(1):82-92, doi:10.29063/ ajrh2017/v21i1.7.

47. WHO, Constitution of the World Health Organization, Apr. 7, 1948, https://www.who.int/governance/eb/who_ constitution_en.pdf.

48. Ghana Health Service (GHS), Prevention and Management of Unsafe Abortion: Comprehensive Abortion Care Services Standards and Protocols, third ed., 2012, https://abortion-policies.srhr.org/documents/countries/02Ghana-Comprehensive-Abortion-Care-Services-Standardsand-Protocols-Ghana-Health-Service-2012.pdf.

49. Daire J, Kloster MO and Storeng KT, Political priority for abortion law reform in Malawi: transnational and national influences, Health and Human Rights Journal, 2018, 20(1):225-236, https://europepmc.org/backend/ ptpmcrender.fcgi?accid=PMC6039725\&blobtype=pdf.

50. Berer M, Abortion law and policy around the world: in search of decriminalization, Health and Human Rights Journal, 2017, 19(1):13-27, PMCID:PMC5473035.

51. Fetters T et al., Moving from legality to reality: how medical abortion methods were introduced with implementation science in Zambia, Reproductive Health, 2017, 14(26), doi:10.1186/s12978-017-0289-2.

52. Cresswell JA et al., Women's knowledge and attitudes surrounding abortion in Zambia: a cross-sectional survey across three provinces, BMJ Open, 2016, 6(3):e010076, doi:10.1136/bmjopen-2015-010076.

53. Jewkes RK et al., Why are women still aborting outside designated facilities in metropolitan South Africa?, BJOG, 2005, 112(9):1236-1242, doi:10.1111/j.14710528.2005.00697.x.

54. Ghana Statistical Service (GSS), GHS and ICF, Ghana Maternal Health Survey 2017, 2018, https://dhsprogram. com/pubs/pdf/FR340/FR340.pdf.

55. GSS, GHS and Macro International, Ghana Maternal Health Survey 2007, 2009, https://dhsprogram.com/pubs/ pdf/FR227/FR227.pdf.

56. WHO, Global Health Observatory data repository: health workforce, medical doctors, 2019, https://apps.who. int/gho/data/node.main.HWFGRP_0020?lang=en.

57. World Bank, Rural population (\% of total population): Sub-Saharan Africa, 2018, https://data.worldbank.org/ indicator/SP.RUR.TOTL.ZS?locations=ZG\&view=chart.

58. Ministry of Health, Standards and Guidelines for Comprehensive Abortion Care in Zambia, Lusaka, Zambia: Government of the Republic of Zambia, 2017, https:// abortion-policies.srhr.org/documents/countries/11Zambia-Standards-and-Guidelines-for-ComprehensiveAbortion-Care-2017.pdf\#page $=38$.

59. Coast $E$ and Murray $S F$, "These things are dangerous": understanding induced abortion trajectories in urban Zambia, Social Science \& Medicine, 2016, 153:201-209, doi:10.1016/j.socscimed.2016.02.025. 
60. Harries J et al., Conscientious objection and its impact on abortion service provision in South Africa: a qualitative study, Reproductive Health, 2014, 11(16), doi:10.1186/17424755-11-16.

61. Awoonor-Williams JK et al., Prevalence of conscientious objection to legal abortion among clinicians in northern Ghana, International Journal of Gynecology \& Obstetrics, 2018, 140(1):31-36, doi:10.1002/ijgo.12328.

62. Freeman $E$ and Coast $E$, Conscientious objection to abortion: Zambian healthcare practitioners' beliefs and practices, Social Science \& Medicine, 2019, 221:106-114, doi:10.1016/j.socscimed.2018.12.018.

63. Family Health Department, Technical and Procedural Guidelines for Safe Abortion Services in Ethiopia, Addis Ababa, Ethiopia: Federal Democratic Republic of Ethiopia, 2006.

64. Federal Ministry of Health, Technical and Procedural Guidelines for Safe Abortion Services in Ethiopia, second ed., Addis Ababa, Ethiopia: Federal Democratic Republic of Ethiopia, 2013, https://abortion-policies.srhr.org/ documents/countries/03-Ethiopia-Technical-andprocedural-guidelines-for-safe-abortion-services-2014.pdf.

65. Gebrehiwot $Y$ et al., Changes in morbidity and abortion care in Ethiopia after legal reform: national results from 2008 and 2014, International Perspectives on Sexual and Reproductive Health, 2016, 42(3):121-130, doi:10.1363/42e1916.

66. Dibaba $Y$ et al., A decade of progress providing safe abortion services in Ethiopia: results of national assessments in 2008 and 2014, BMC Pregnancy and Childbirth, 2017, 17(76), doi:10.1186/s12884-017-1266-z.

67. Republic of Ghana, Criminal Code (Amendment), Law 102, 1985.

68. Damalie FJMK et al., Severe morbidities associated with induced abortions among misoprostol users and non-users in a tertiary public hospital in Ghana, BMC Women's Health, 2014, 14(90), doi:10.1186/1472-6874-14-90.

69. Otupiri E, Kwame Nkrumah University of Science and Technology, Kumasi, Ghana, personal communication, Nov. 20, 2019.

70. Owolabi $\mathrm{OO}$ et al., Incidence of abortion-related near-miss complications in Zambia: cross-sectional study in Central, Copperbelt and Lusaka Provinces, Contraception, 2017, 95(2):167-174, doi:10.1016/j. contraception.2016.08.014.

71. Geary CW et al., Attitudes toward abortion in Zambia, International Journal of Gynecology \& Obstetrics, 2012, 118:S148-S151, doi:10.1016/S0020-7292(12)60014-9.

72. Special tabulations of data from Bearak JM et al., Unintended pregnancy and abortion by income, region, and the legal status of abortion: estimates from a comprehensive model for 1990-2019, Lancet Global Health, 2020, 8(9):E1152-E1161, doi:https://doi.org/10.1016/S2214109X(20)30315-6.

73. Singh $S$, Bankole A and Darroch JE, The impact of contraceptive use and abortion on fertility in subSaharan Africa: estimates for 2003-2014, Population and Development Review, 2017, 43(Suppl. 1):141-165, doi:10.1111/padr.12027.

74. Shelton JD, Taking exception. Reduced mortality leads to population growth: an inconvenient truth, Global Health:
Science and Practice, 2014, 2(2):135-138, doi:10.9745/ GHSP-D-14-00062.

75. Tabutin $D$ and Schoumaker $B$, The demography of sub-Saharan Africa from the 1950s to the 2000s: a survey of changes and a statistical assessment, Population, 2004, 59(3):455-555, doi:https://www.cairn-int.info/revuepopulation-2004-3-page-455.htm.

76. Sully EA et al., Abortion in Zimbabwe: a national study of the incidence of induced abortion, unintended pregnancy and post-abortion care in 2016, PLOS ONE, 2018, 13(10):e0205239, doi:10.1371/journal.pone.0205239.

77. Keogh SC et al., Estimating the incidence of abortion: a comparison of five approaches in Ghana, BMJ Global Health, 2020, 5(4):e002129, doi:10.1136/bmjgh-2019-002129.

78. Garenne M, A century of nuptiality decline in South Africa: a longitudinal analysis of census data, African Population Studies, 2016, 30(2):2403-2414, doi:10.11564/ 30-2-846.

79. Special tabulations of data from the 2008 application of the abortion incidence complications method and Community-Based Survey in Burkina Faso.

80. Sully EA et al., Estimating abortion incidence among adolescents and differences in postabortion care by age: a cross-sectional study of postabortion care patients in Uganda, Contraception, 2018, 98(6):510-516, doi:10.1016/ j.contraception.2018.07.135.

81. Special tabulations of data from the 2012 application of the abortion incidence complications method and Prospective Morbidity Survey in Kenya.

82. Sully E et al., Playing it safe: legal and clandestine abortions among adolescents in Ethiopia, Journal of Adolescent Health, 2018, 62(6):729-736, doi:10.1016/ j.jadohealth.2017.12.015.

83. Bongaarts J, Mensch BS and Blanc AK, Trends in the age at reproductive transitions in the developing world: the role of education, Population Studies, 2017, 71(2):139-154, doi:10.1080/00324728.2017.1291986.

84. Sully E et al., Adding It Up: Investing in Sexual and Reproductive Health 2019-Appendix Tables, New York: Guttmacher Institute, 2020, https://www.guttmacher.org/ report/adding-it-up-investing-in-sexual-reproductivehealth-2019.

85. Assemblée Nationale du Bénin, Code de l'Enfant en République du Bénin, Loi n 2015-08, Jan. 23, 2015.

86. Assemblée Nationale de la République Centrafricaine, Loi №10.001 du 06 janvier 2010 portant Code Pénal Centrafricaine, Journal Officiel de la République Centrafricaine, 2010, Edition Spéciale.

87. Government of Eritrea, Penal Code of the State of Eritrea, 2015.

88. Assemblée Nationale et Sénat de Gabon, Loi n042/2018 du 05 juillet 2019 portant Code Pénal, Journal Officiel de la République Gabonaise, 2019, No. 27, Bis Spécial.

89. Assemblée Nationale de la République de Guinée, Code Pénal de la République de Guinée, Loi N ${ }^{\circ}$ 98/036, Dec. 31, 1998.

90. Ministério da Saúde, Diploma Ministerial, No. 60/2017, Boletim da República, Publicação Oficial da República de Moçambique, 2017, I SÉRIE(Número 147):1111-1141. 
91. DHS Program, STATcompiler: Use of traditional contraception, and unmet need for contraception, among married women and sexually active unmarried women, adolescents and women aged 15-49, Sub-Saharan African countries with data, 2007-2018, https://www.statcompiler. com/en/.

92. Hall KS et al., Bad girl and unmet family planning need among Sub-Saharan African adolescents: the role of sexual and reproductive health stigma, Qualitative Research in Medicine \& Healthcare, 2018, 2(1):55-64, doi:10.4081/ qrmh.2018.7062.

93. Francis $E$ and Gabriel VC, Barriers limiting youth access to reproductive health services by primary health care facilities in Nigeria, Universal Journal of Public Health, 2019, 7(1):36-43, doi:10.13189/ujph.2019.070105.

94. Sedgh G, Ashford LS and Hussain R, Unmet Need for Contraception in Developing Countries: Examining Women's Reasons for Not Using a Method, New York: Guttmacher Institute, 2016, https://www.guttmacher.org/sites/default/ files/report_pdf/unmet-need-for-contraception-indeveloping-countries-report.pdf.

95. Yegon EK et al., Understanding abortion-related stigma and incidence of unsafe abortion: experiences from community members in Machakos and Trans Nzoia counties Kenya, Pan African Medical Journal, 2016, 24(258), doi:10.11604/pamj.2016.24.258.7567.

96. Appiah-Agyekum N, Abortions in Ghana: experiences of university students, Health Science Journal, 2014, 8(4):531-540.

97. Lupwana K, Les Avortements clandestins et à risque des adolescentes par l'automédication dans les périphériques de Kinshasa: facteurs de vulnérabilité, Department of International Law, University of Johannesburg, no date.

98. Bell SO et al., Inequities in the incidence and safety of abortion in Nigeria, BMJ Global Health, 2020, 5:e001814, doi:10.1136/bmjgh-2019-001814.

99. Paluku L et al., Knowledge and attitude of schoolgirls about illegal abortions in Goma, Democratic Republic of Congo, African Journal of Primary Health Care \& Family Medicine, 2010, 2(1), doi:10.4102/phcfm.v2i1.78.

100. Casey SE et al., "You must first save her life": community perceptions towards induced abortion and post-abortion care in North and South Kivu, Democratic Republic of the Congo, Sexual and Reproductive Health Matters, 2019, 27(1):106-117, doi:10.1080/09688080.2019. 1571309.

101. Boah $M$, Bordotsiah $S$ and Kuurdong $S$, Predictors of unsafe induced abortion among women in Ghana, Journal of Pregnancy, 2019, doi:10.1155/2019/9253650.

102. Baxerres $C$ et al., Abortion in two francophone African countries: a study of whether women have begun to use misoprostol in Benin and Burkina Faso, Contraception, 2018, 97(2):130-136, doi:10.1016/j.contraception.2017.10.011.

103. Mutua MM et al., Factors associated with delays in seeking post abortion care among women in Kenya, $B M C$ Pregnancy and Childbirth, 2015, 15(241), doi:10.1186/ s12884-015-0660-7.

104. Abiodun $O$ et al., Complications of unsafe abortion in South West Nigeria: a review of 96 cases, African Journal of Medicine and Medical Sciences, 42(1):111-115.

105. Ishoso D, Tshefu AK and Coppieters Y, Analysis of in- duced abortion-related complications in women admitted to the Kinshasa reference general hospital: a tertiary health facility, Democratic Republic of the Congo, Reproductive Health, 2018, 15(123), doi:10.1186/s12978-018-0563-y.

106. Ishoso DK et al., Analysis of induced abortion-related complications in women admitted to referral health facilities in Kinshasa, Democratic Republic of the Congo, PLOS ONE, 2018, 13(8):e0203186, doi:10.1371/journal. pone.0203186.

107. Ushie BA et al., Timing of abortion among adolescent and young women presenting for post-abortion care in Kenya: a cross-sectional analysis of nationallyrepresentative data, BMC Women's Health, 2018, 18(41), doi:10.1186/s12905-018-0521-4.

108. Madziyire MG et al., Severity and management of postabortion complications among women in Zimbabwe, 2016: a cross-sectional study, BMJ Open, 2018, 8(2):e019658, doi:10.1136/bmjopen-2017-019658.

109. Ziraba AK et al., Unsafe abortion in Kenya: a crosssectional study of abortion complication severity and associated factors, BMC Pregnancy and Childbirth, 2015, 15(34), doi:10.1186/s12884-015-0459-6.

110. Kalilani-Phiri L et al., The severity of abortion complications in Malawi, International Journal of Gynecology \& Obstetrics, 2015, 128(2):160-164, doi:10.1016/ j.ijgo.2014.08.022.

111. Bankole $A$ et al., The severity and management of complications among postabortion patients treated in Kinshasa health facilities, International Perspectives on Sexual and Reproductive Health, 2018, 44(1):1-9, doi:10.1363/44e5618.

112. Payne $C$ et al., Why women are dying from unsafe abortion: narratives of Ghanaian abortion providers, African Journal of Reproductive Health, 2013, 17(2):118-128, PMID: 24069757.

113. Chae $S$ et al., Characteristics of women obtaining induced abortions in selected low- and middle-income countries, PLOS ONE, 2017, 12(3):e0172976, doi:10.1371/ journal.pone.0172976.

114. DHS Program, STATcompiler: Wanted total fertility rates and ideal family size by area of residence, educational level and wealth quintile, Sub-Saharan African countries with data, 2007-2018, https://www.statcompiler.com/en/.

115. Atake E-H and Gnakou Ali P, Women's empowerment and fertility preferences in high fertility countries in Sub-Saharan Africa, BMC Women's Health, 2019, 19(54), doi:10.1186/s12905-019-0747-9.

116. Moore AM et al., The estimated incidence of induced abortion in Ethiopia, 2014: changes in the provision of services since 2008, International Perspectives on Sexual and Reproductive Health, 2016, 42(3):111-120, doi:10.1363/42e1816.

117. Special tabulations of data from Sedgh $\mathrm{G}$ et al., Estimates of the incidence of induced abortion and consequences of unsafe abortion in Senegal, International Perspectives on Sexual and Reproductive Health, 2015, 41(1):11-19, doi:10.1363/4101115.

118. Chae $S$ et al., The incidence of induced abortion in Kinshasa, Democratic Republic of Congo, 2016, PLOS ONE, 2017, 12(10):e0184389, doi:10.1371/journal.pone.0184389.

119. Ganle JK et al., Disparities in abortion experience and 
access to safe abortion services in Ghana: evidence from a retrospective survey, African Journal of Reproductive Health, 2016, 20(2):43-52, doi:10.29063/ajrh2016/v20i2.5.

120. Bénié Bi Vroh J et al., Épidémiologie des avortements provoqués en Côte d'Ivoire, Santé Publique, 2012, 24(Hors Série):67-76, doi:10.3917/spub.120.0067.

121. Médecins du Monde, Les déterminants socioculturels et communautaires des grossesses non désirées et des avortements, Ville de Kinshasa, République Démocratique du Congo, 2015, https://www.medecinsdumonde.org/ fr/actualites/publications/2015/11/30/les-determinantssocioculturels-et-communautaires-des-grossesses-nondesirees-et-des-avortements.

122. Lince-Deroche $\mathrm{N}$ et al., Unintended Pregnancy and Abortion in Kinshasa, Democratic Republic of Congo: Challenges and Progress, New York: Guttmacher Institute, 2019, https://www.guttmacher.org/report/unintendedpregnancy-abortion-kinshasa-drc

123. Foumsou $L$ et al., Problematic of clandestine induced abortions in three maternity hospitals of Chad, Open Journal of Obstetrics and Gynecology, 2017, 7(9):937-943, doi:10.4236/ojog.2017.79094.

124. Chae $S$ et al., Reasons why women have induced abortions: a synthesis of findings from 14 countries, Contraception, 2017, 96(4):233-241, doi:10.1016/ j.contraception.2017.06.014

125. Konney TO et al., Attitude of women with abortionrelated complications toward provision of safe abortion services in Ghana, Journal of Women's Health, 2009, 18(11):1863-1866, doi:10.1089/jwh.2008.1190.

126. Mote $\mathrm{CV}$ et al., Factors associated with induced abortion among women in Hohoe, Ghana, African Journal of Reproductive Health, 2010, 14(4):110-116.

127. Direction Générale de la Statistique (DGS) and ICF International, Enquête Démographique et de Santé du Gabon 2012, 2013, https://dhsprogram.com/pubs/pdf/FR276/ FR276.pdf.

128. Special tabulations of data on women's main reason for abortion from reference 54 .

129. Centre Nationale de la Statistique et des Études Économiques and ICF International, Enquête Démographique et de Santé du Congo 2011-2012, 2013, https://dhsprogram.com/pubs/pdf/FR267/FR267.pdf.

130. National Academies of Sciences, Engineering, and Medicine, The Safety and Quality of Abortion Care in the United States, Washington, DC: National Academies Press, 2018, doi:10.17226/24950.

131. WHO, Clinical Practice Handbook for Safe Abortion, 2014, https://apps.who.int/iris/bitstream/ handle/10665/97415/9789241548717_eng.pdf;jsessionid=E22B5FA7DA0BD8216309B956A9BE2996? sequence $=1$.

132. Raymond EG and Grimes DA, The comparative safety of legal induced abortion and childbirth in the United States, Obstetrics and Gynecology, 2012, 119 (2 pt. 1):215219, doi:10.1097/AOG.0b013e31823fe923.

133. Parmar $\mathrm{D}$ et al., Cost of abortions in Zambia: a comparison of safe abortion and post abortion care, Global Public Health, 2017, 12(2):236-249, doi:10.1080/17441692.2015.1 123747.

134. Special tabulations of data from the 2014 Prospective Morbidity Survey, Ethiopia.
135. Lince-Deroche $\mathrm{N}$ et al., Doing more for less: identifying opportunities to expand public sector access to safe abortion in South Africa through budget impact analysis, Contraception, 2018, 97(2):167-176, doi:10.1016/ j.contraception.2017.07.165.

136. Norris $A$ et al., Abortion experiences among Zanzibari women: a chain-referral sampling study, Reproductive Health, 2016, 13(23), doi:10.1186/s12978-016-0129-9.

137. Harris LH and Grossman D, Confronting the challenge of unsafe second-trimester abortion, International Journal of Gynecology \& Obstetrics, 2011, 115(1):77-79, doi:10.1016/j.ijgo.2011.05.018.

138. Altshuler AL et al., Male partners' involvement in abortion care: a mixed-methods systematic review: men's roles in clinical abortion care, Perspectives on Sexual and Reproductive Health, 2016, 48(4):209-219, doi:10.1363/ psrh.12000.

139. Schwandt HM et al., Pathways to unsafe abortion in Ghana: the role of male partners, women and health care providers, Contraception, 2013, 88(4):509-517, doi:10.1016/j.contraception.2013.03.010.

140. Freeman E, Coast E and Murray S, Men's roles in women's abortion trajectories in urban Zambia, International Perspectives on Sexual and Reproductive Health 2017, 43(2):89-98, doi:10.1363/43e4017.

141. Moore AM, Jagwe-Wadda $G$ and Bankole $A$, Men's attitudes about abortion in Uganda, Journal of Biosocial Science, 2011, 43(1):31-45, doi:10.1017/ S0021932010000507.

142. Marlow HM et al., Women's perceptions about abortion in their communities: perspectives from western Kenya, Reproductive Health Matters, 2014, 22(43):149-158, doi:10.1016/S0968-8080(14)43758-3.

143. Jackson E et al., A strategic assessment of unsafe abortion in Malawi, Reproductive Health Matters, 2011, 19(37):133-143.

144. Gerdts $C$ et al., Women's experiences seeking informal sector abortion services in Cape Town, South Africa: a descriptive study, BMC Women's Health, 2017, 17(95), doi:10.1186/s12905-017-0443-6.

145. AdMinFem, Les ados et grossesses non désirées au Congo, Si jeunesse savait: pour que la choix de jeunesse compte, 2015, http://mwasi.com/conference-debat-surla-problematique-des-grossesses-non-desirees-chez-lesadolescents-et-jeunes-en-rd-congo.

146. Médecins du Monde, Les déterminants socioculturels et communautaires des grossesses non désirées et des avortements, District de Djibo, Burkina Faso, 2015.

147. PMA 2020, PMA2020 Abortion Survey Results: Côte d'Ivoire, July-August 2018, https://www.pma2020. org/sites/default/files/Cote\%20d\%E2\%80\%99lvoire_ AbortionModule_Brief_EN_012019.pdf.

148. WHO, Safe Abortion: Technical and Policy Guidance for Health Systems. Evidence Summaries and Grade Tables, 2012, No. WHO/RHR/12.10, https://apps.who.int/iris/ bitstream/handle/10665/75840/WHO_RHR_12.10_eng. pdf? sequence $=1$.

149. WHO, Medical Management of Abortion, 2018, https:// www.who.int/reproductivehealth/publications/medicalmanagement-abortion/en/.

150. Raymond EG, Harrison MS and Weaver MA, Efficacy 
of misoprostol alone for first-trimester medical abortion: a systematic review, Obstetrics \& Gynecology, 2019, 133(1):137-147, doi:10.1097/AOG.0000000000003017.

151. Appiah-Agyekum NN, Medical abortions among university students in Ghana: implications for reproductive health education and management, International Journal of Women's Health, 2018, 10:515-522, doi:10.2147/JJWH. S160297.

152. Dah T et al., Introducing misoprostol for the treatment of incomplete abortion in Nigeria, African Journal of Reproductive Health, 2011, 15(4):42-50.

153. International Federation of Gynecology and Obstetrics (FIGO), Misoprostol-Only Recommended Regimens 2017, no date, https://www.figo.org/sites/default/files/2020-06/ FIGO\%20Dosage\%20Chart\%20\%20-\%20ENGLISH.pdf.

154. Okonofua F, Misoprostol and women's health in Africa, editorial, African Journal of Reproductive Health, 2005, 9(1):7-9, https://www.ajrh.info/index.php/ajrh/article/ view/1118.

155. Zamberlin N, Romero M and Ramos S, Latin American women's experiences with medical abortion in settings where abortion is legally restricted, Reproductive Health 2012, 9:34, doi:10.1186/1742-4755-9-34.

156. Hall $P$ and Tagontong N, Quality of misoprostol products, WHO Drug Information, 2016, 30(1):35-39, https:// www.who.int/medicines/publications/druginformation/ WHO_DI_30-1_Quality.pdf?ua=1.

157. Anyakora $C$ et al., Quality medicines in maternal health: results of oxytocin, misoprostol, magnesium sulfate and calcium gluconate quality audits, BMC Pregnancy and Childbirth, 2018, 18(44), doi:10.1186/s12884-018-1671-y.

158. Berard $V$ et al., Instability of misoprostol tablets stored outside the blister: a potential serious concern for clinical outcome in medical abortion, PLOS ONE, 2014, 9(12):e112401, doi:10.1371/journal.pone.0112401.

159. Rominski SD, Lori JR and Morhe ES, "My friend who bought it for me, she has had an abortion before." The influence of Ghanaian women's social networks in determining the pathway to induced abortion, Journal of Family Planning and Reproductive Health Care, 2017, 43(3):216-221, doi:10.1136/jfprhc-2016-101502.

160. Bello FA et al., Trends in misoprostol use and abortion complications: a cross-sectional study from nine referral hospitals in Nigeria, PLOS ONE, 2018, 13(12):e0209415, doi:10.1371/journal.pone.0209415.

161. Solheim IH et al., Beyond the law: misoprostol and medical abortion in Dar es Salaam, Tanzania, Social Science \& Medicine, 2020, 245:112676, doi:10.1016/ j.socscimed.2019.112676.

162. Footman $\mathrm{K}$ et al., Medical abortion provision by pharmacies and drug sellers in low- and middle-income countries: a systematic review, Studies in Family Planning, 2018, 49(1):57-70, doi:10.1111/sifp.12049.

163. Reiss K et al., Pharmacy workers' knowledge and provision of medication for termination of pregnancy in Kenya, Journal of Family Planning and Reproductive Health Care, 2016, 42(3):208-212, doi:10.1136/jfprhc-2013-100821.

164. Hendrickson C et al., Client-pharmacy worker interactions regarding medical abortion in Zambia in 2009 and 2011, International Journal of Gynecology \& Obstetrics, 2016, 132(2):214-218, doi:10.1016/j.ijgo.2015.07.008.
165. Special tabulations of data from Health Professionals Surveys conducted in Burkina Faso (2008), Kenya (2012), Malawi (2015), Nigeria (2012), Rwanda (2009), Senegal (2012), Tanzania (2013), Uganda (2013) and Zimbabwe (2016).

166. Ganatra B et al., From concept to measurement: operationalizing WHO's definition of unsafe abortion, Bulletin of the World Health Organization, 2014, 92(3):155, http:// dx.doi.org/10.2471/BLT.14.136333.

167. Sedgh $\mathrm{G}$ et al., Insights from an expert group meeting on the definition and measurement of unsafe abortion, International Journal of Gynecology \& Obstetrics, 2016, 134(1):104-106, doi:10.1016/j.ijgo.2015.11.017.

168. FIGO Ethical and Professional Aspects of Human Reproduction and Women's Health Committee, FIGO committee statement: ethical responsibilities in post-abortion care, International Journal of Gynecology \& Obstetrics, 2019, 146(3):269-270, doi:10.1002/ijgo.12899.

169. Singh $S$ and Maddow-Zimet I, Facility-based treatment for medical complications resulting from unsafe pregnancy termination in the developing world, 2012: a review of evidence from 26 countries, BJOG, 2016, 123(9):1489-1498, doi:10.1111/1471-0528.13552.

170. Pourette D et al., Complications with use of misoprostol for abortion in Madagascar: between ease of access and lack of information, Contraception, 2018, 97(2):116-121, doi:10.1016/j.contraception.2017.12.005.

171. Moore AM et al., Comparing women's financial costs of induced abortion at a facility vs. seeking treatment for complications from unsafe abortion in Zambia, Reproductive Health Matters, 2018, 26(52):138-150, doi:10.1080/09688080.2018.1522195.

172. Special tabulations of data from Prospective Morbidity Surveys conducted in Ethiopia (2008), Kenya (2012), Malawi (2009), Uganda (2013) and Zimbabwe (2016).

173. Benson J et al., Costs of postabortion care in public sector health facilities in Malawi: a cross-sectional survey, BMC Health Services Research, 2015, 15(562), doi:10.1186/ s12913-015-1216-2.

174. Paul $M$ et al., Unsafe abortion in Sierra Leone: an examination of costs and burden of treatment on healthcare resources, Journal of Women's Health Care, 2015, 4(2):228, doi:10.4172/2167-0420.1000228.

175. Rehnström Loi $U$ et al., Health care providers' perceptions of and attitudes towards induced abortions in sub-Saharan Africa and Southeast Asia: a systematic literature review of qualitative and quantitative data, $B M C$ Public Health, 2015, 15(139), doi:10.1186/s12889-015-1502-2.

176. Chiweshe M and Macleod C, "If you choose to abort, you have acted as an instrument of Satan": Zimbabwean health service providers' negative constructions of women presenting for post abortion care, International Journal of Behavioral Medicine, 2017, 24(6):856-863, doi:10.1007/ s12529-017-9694-8.

177. Storeng KT and Ouattara F, The politics of unsafe abortion in Burkina Faso: the interface of local norms and global public health practice, Global Public Health, 2014, 9(8):946-959, doi:10.1080/17441692.2014.937828.

178. Kumar A, Hessini L and Mitchell EMH, Conceptualising abortion stigma, Culture, Health \& Sexuality, 2009, 11(6):625-639, doi:10.1080/13691050902842741. 
179. Levandowski B et al., Investigating social consequences of unwanted pregnancy and unsafe abortion in Malawi: the role of stigma, International Journal of Gynecology \& Obstetrics, 2012, 118(Suppl. 2):S167-S171, doi:10.1016/ S0020-7292(12)60017-4.

180. Mayi-Tsonga $S$ et al., Delay in the provision of adequate care to women who died from abortion-related complications in the principal maternity hospital of Gabon, Reproductive Health Matters, 2009, 17(34):65-70, doi:10.1016/S0968-8080(09)34465-1.

181. Melese T et al., Management of post abortion complications in Botswana-the need for a standardized approach, PLOS ONE, 2018, 13(2):e0192438, doi:10.1371/ journal.pone.0192438.

182. Berhan $Y$ and Berhan $A$, Causes of maternal mortality in Ethiopia: a significant decline in abortion related death, Ethiopian Journal of Health Sciences, 2014, Special Issue, pp. 17-28, doi:10.4314/ejhs.v24i0.3S.

183. WHO, Health Worker Roles in Providing Safe Abortion Care and Post-Abortion Contraception, 2015, https://apps. who.int/iris/bitstream/handle/10665/181041/ 9789241549264_eng.pdf?sequence $=1$.

184. Makenzius $M$ et al., Post-abortion care with misoprostol-equally effective, safe and accepted when administered by midwives compared to physicians: a randomised controlled equivalence trial in a lowresource setting in Kenya, BMJ Open, 2017, 7(10):e016157, doi:10.1136/bmjopen-2017-016157.

185. Gaye A et al., Decentralizing postabortion care in Senegal with misoprostol for incomplete abortion, International Journal of Gynecology \& Obstetrics, 2014, 126(3):223-226, doi:10.1016/j.ijgo.2014.03.028.

186. Maternowska MC et al., Perceptions of misoprostol among providers and women seeking post-abortion care in Zimbabwe, Reproductive Health Matters, 2014, 22(Suppl. 44):16-25, doi:10.1016/S0968-8080(14)43792-3.

187. Mayi-Tsonga $S$ et al., The contribution of research results to dramatic improvements in post-abortion care: Centre Hospitalier de Libreville, Gabon, Reproductive Health Matters, 2012, 20(40):16-21, doi:10.1016/S09688080(12)40670-X.

188. Cook S, de Kok B and Odland ML, "It's a very complicated issue here": understanding the limited and declining use of manual vacuum aspiration for postabortion care in Malawi: a qualitative study, Health Policy and Planning, 2017, 32(3):305-313, doi:10.1093/heapol/czw128.

189. Odland ML et al., Decrease in use of manual vacuum aspiration in postabortion care in Malawi: a cross-sectional study from three public hospitals, 2008-2012, PLOS ONE, 2014, 9(6):e100728, doi:10.1371/journal.pone.0100728.

190. Owolabi OO, Biddlecom A and Whitehead HS, Health systems' capacity to provide post-abortion care: a multicountry analysis using signal functions, Lancet Global Health, 2019, 7:e110-e118, doi:10.1016/S2214109X(18)30404-2.

191. Benson J et al., What contraception do women use after abortion? An analysis of 319,385 cases from eight countries, Global Public Health, 2018, 13(1):35-50, doi:10.1080/17441692.2016.1174280.

192. Evens $E$ et al., Post-abortion care services for youth and adult clients in Kenya: a comparison of services, client satisfaction and provider attitudes, Journal of Biosocial Science, 2014, 46:1-15, doi:10.1017/S0021932013000230.

193. Prata $\mathrm{N}$ et al., Comprehensive Abortion Care Pilot Project in Tigray, Ethiopia: Final Report, Tigray, Ethiopia: Tigray Regional Health Bureau, Ventura Strategies Innovations and Bixby Center, 2011, http://bixby.berkeley.edu/wp-content/ uploads/2015/03/TRHB_VSI_Bixby-Ethiopia-CAC-PilotFinal-Report-2011-05F.pdf.

194. Tsui AO, Brown W and Li Q, Contraceptive practice in sub-Saharan Africa, Population and Development Review, 2017, 43(Suppl 1):166-191, doi:10.1111/padr.12051.

195. Sundaram A et al., Documenting the individualand household-level cost of unsafe abortion in Uganda, International Perspectives on Sexual and Reproductive Health, 2013, 39(4):174-184, doi:10.1363/3917413.

196. Ilboudo PGC et al., Estimating the costs for the treatment of abortion complications in two public referral hospitals: a cross-sectional study in Ouagadougou, Burkina Faso, BMC Health Services Research, 2016, 16(559), doi:10.1186/s12913-016-1822-7.

197. Hu D et al., Cost-effectiveness analysis of unsafe abortion and alternative first-trimester pregnancy termination strategies in Nigeria and Ghana, African Journal of Reproductive Health, 2010, 14(2):85-103.

198. WHO, Report of a WHO Technical Consultation on Birth Spacing, 2005, https://apps.who.int/iris/handle/ 10665/69855.

199. The Alan Guttmacher Institute (AGI), Sharing Responsibility: Women, Society and Abortion Worldwide, 1999, https://www.guttmacher.org/sites/default/files/pdfs/ pubs/sharing.pdf.

200. Bongaarts J, Africa's unique fertility transition, Population and Development Review, 2017, 43(S1):39-58, doi:10.1111/j.1728-4457.2016.00164.x.

201. INSEED, Ministère de la Santé Publique and ICF International, Enquête Démographique et de Santé et à Indicateurs Multiples au Tchad, 2014-2015, 2016, https:// www.dhsprogram.com/pubs/pdf/FR317/FR317.pdf.

202. Institut National de la Statistique and ICF International, Enquête Démographique et de Santé et à Indicateurs Multiples du Niger, 2012, 2013, https://dhsprogram.com/ pubs/pdf/FR277/FR277.pdf.

203. Trussell J and Aiken ARA, Contraceptive efficacy, in: Hatcher R et al., eds., Contraceptive Technology, 21st ed., New York: Ayer Company Publishers, 2018, pp. 829-839.

204. Department of Economic and Social Affairs, UN Population Division, Estimates and Projections of Family Planning Indicators 2020, 2020, https://www.un.org/en/ development/desa/population/theme/family-planning/ cp_model.asp.

205. DHS Program, STATcompiler: Use of modern contraception among married women and sexually active unmarried women, Sub-Saharan African countries with data, 2007-2018, https://www.statcompiler.com/en/.

206. Thompson J, Undie C and Askew I, Access to emergency contraception and safe abortion services for survivors of rape: a review of policies, programmes and country experiences in sub-Saharan Africa, Step-Up Research Report, Nairobi, Kenya: Population Council, 2014, https://www.svri. org/sites/default/files/attachments/2016-07-15/2014STEPUP_EC-SA_Report.pdf. 
207. Inter-Agency Working Group on Reproductive Health in Crisis Situations, Manual: Inter-Agency Reproductive Health Kits for Crisis Situations, fifth ed., New York: United Nations Population Fund, 2011, https://www.unfpa.org/ sites/default/files/resource-pdf/RH\%20kits\%20manual_ EN_0.pdf.

208. Barot $S$, In a state of crisis: meeting the sexual and reproductive health needs of women in humanitarian situations, Guttmacher Policy Review, 2017, 20:24-30, https://www.guttmacher.org/gpr/2017/02/state-crisismeeting-sexual-and-reproductive-health-needs-womenhumanitarian-situations.

209. Kisindja RM et al., Family planning knowledge and use among women in camps for internally displaced people in the Democratic Republic of the Congo, International Journal of Gynecology \& Obstetrics, 2017, 138(3):256-260, doi:10.1002/ijgo.12220.

210. Chukwumalu K et al., Uptake of postabortion care services and acceptance of postabortion contraception in Puntland, Somalia, Reproductive Health Matters, 2017, 25(51):48-57, doi:10.1080/09688080.2017.1402670.

211. Jayaweera RT et al., Women's experiences with unplanned pregnancy and abortion in Kenya: a qualitative study, PLOS ONE, 2018, 13(1):e0191412, doi:10.1371/journal. pone.0191412.

212. Department of Economic and Social Affairs, UN Population Division, Estimates and Projections of Women of Reproductive Age Who Are Married or in a Union: 2020 Revision, 2020, https://www.un.org/en/development/desa/ population/theme/marriage-unions/marriage_estimates. asp.

213. Bonnen KI, Tuijje DN and Rasch V, Determinants of first and second trimester induced abortion-results from a cross-sectional study taken place 7 years after abortion law revisions in Ethiopia, BMC Pregnancy and Childbirth, 2014, 14(416), doi:10.1186/s12884-014-0416-9.

214. Esia-Donkoh K et al., Who cares? Pre and post abortion experiences among young females in Cape Coast metropolis, Ghana, African Journal of Reproductive Health, 2015, 19(2):43-51, doi:http://www.jstor.org/ stable/24877769.

215. United Nations Children's Fund, Child Marriage: Latest Trends and Future Prospects, 2018, https://data.unicef. org/resources/child-marriage-latest-trends-and-futureprospects/.

216. World Bank, Secondary school enrollment, female (\% net), Sub-Saharan Africa, https://data.worldbank.org/ indicator/SE.SEC.NENR.FE?locations=ZG.

217. DHS Program, STATcompiler: Median age at first sex and median age at first marriage, Sub-Saharan African countries with data, 2007-2018, https://www.statcompiler. com/en/.

218. WHO et al., Trends in Maternal Mortality: 2000 to 2017: Estimates by WHO, UNICEF, UNFPA, World Bank Group and the United Nations Population Division, 2019, http://www.who. int/reproductivehealth/publications/monitoring/maternalmortality-2015/en/.

219. Pan American Health Organization, Changing Relationships in the Health Care Context: The Uruguayan Model for Reducing the Risk and Harm of Unsafe Abortion, 2012, https://www.paho.org/hq/dmdocuments/2013/ Publication-EN-BP-Uruguay-2012.pdf.
220. Benson J et al., Improving health worker performance of abortion services: an assessment of post-training support to providers in India, Nepal and Nigeria, Reproductive Health, 2017, 14(154), doi:10.1186/s12978-017-0416-0.

221. Bart Johnson $\mathrm{H}$ and Westoff $C$, Examples of modelbased approaches to estimating abortion, in: Singh S, Remez L and Tartaglione A, eds., Methodologies for Estimating Abortion Incidence and Abortion-Related Morbidity: A Review, New York: Guttmacher Institute; and Paris: International Union for the Scientific Study of Population, 2010, pp. 49-61, https://www.guttmacher.org/ sites/default/files/report_pdf/abortion-methodologies.pdf.

222. Bongaarts J and Potter R, Fertility, Biology, and Behavior: An Analysis of the Proximate Determinants, New York: Academic Press, 1983.

223. Singh $S$ et al., Estimating abortion incidence: assessment of a widely used indirect method, Population Research and Policy Review, 2019, 38:429-458, doi:10.1007/s11113019-09517-2.

224. Special tabulations of data from Basinga $P$ et al., Abortion incidence and postabortion care in Rwanda, Studies in Family Planning, 2012, 43(1):11-20, doi:10.1111/ j.1728-4465.2012.00298.x.

225. UN Development Programme, Gender Inequality Index (GII): Human Development Reports, no date, http:// hdr.undp.org/en/content/gender-inequality-index-gii.

226. CRR, Abortion worldwide: 20 years of reform, Briefing Paper, 2014, https://www.reproductiverights.org/sites/crr. civicactions.net/files/documents/20Years_Reform_Report. pdf.

227. Remez L, Mayall K and Singh S, Global developments in laws on induced abortion: 2008-2019, International Perspectives on Sexual and Reproductive Health, 2020, 46(Suppl.1):53-65, https://doi.org/10.1363/46e0920.

228. Riley T et al., Adding It Up: Investing in Sexual and Reproductive Health, 2019-Methodology Report, New York: Guttmacher Institute, 2020, https://www.guttmacher.org/ report/adding-it-up-investing-in-sexual-reproductivehealth-2019-methodology.

229. Institute for Health Metrics and Evaluation (IHME), Global Burden of Disease Study 2017 results, 2018, http:// ghdx.healthdata.org/gbd-results-tool.

230. Áhman E and Shah $\mathrm{IH}, \mathrm{New}$ estimates and trends regarding unsafe abortion mortality, International Journal of Gynecology \& Obstetrics, 2011, 115(2):121-126, doi:10.1016/j.ijgo.2011.05.027. 
From Unsafe to Safe Abortion in Sub-Saharan Africa: Slow but Steady Progress is available online. To download, visit https://www.guttmacher.org/report/ from-unsafe-to-safe-abortion-in-subsaharan-africa.

(C2020 Guttmacher Institute, a not-for-profit corporation advancing sexual and reproductive health worldwide through research, policy analysis and public education.

All rights, including translation into other languages, reserved under the Universal Copyright Convention, the Berne Convention for the Protection of Literary and Artistic Works and the Inter- and Pan American Copyright Conventions (Mexico City and Buenos Aires). Rights to translate information contained in this report may be waived.
ISBN: 978-1-934387-23-8

Suggested citation: Bankole A et al., From Unsafe to Safe Abortion in Sub-Saharan Africa: Slow but Steady Progress, New York: Guttmacher Institute, 2020, https://www.guttmacher.org/report/ from-unsafe-to-safe-abortion-in-subsaharan-africa. DOI: $10.1363 / 2020.32446$

Guttmacher Institute 125 Maiden Lane, 7th Floor New York, NY 10038 Telephone: +1-212-248-1111

Fax: +1-212-248-1951

Email: info@guttmacher.org

www.guttmacher.org 
